\title{
Real Forms of the Radon-Penrose Transform
}

\author{
By \\ Andrea D'Agnolo* and Corrado MARASTONI**
}

\begin{abstract}
The complex Radon correspondence relates an $n$-dimensional projective space with the Grassmann manifold of its $p$-dimensional planes. This is the geometric background of the Radon-Penrose transform, which intertwines cohomology classes of homogeneous line bundles with holomorphic solutions to the generalized massless field equations. A good framework to deal with such problems is provided by the recently developed theory of integral transforms for sheaves and $\mathcal{D}$-modules. In particular, an adjunction formula describes the range of transforms acting on general function spaces, associated with constructible sheaves.

The linear group $S L(n+1, \mathbf{C})$ naturally acts on the Radon correspondence. A distinguished family of function spaces is then the one associated with locally constant sheaves along the closed orbits of the real forms of $S L(n+1, \mathbf{C})$. In this paper, we systematically apply the above-mentioned adjunction formula to such function spaces. We thus obtain in a unified manner several results concerning the complex, conformal, or real Radon transforms.
\end{abstract}

\section{Contents}

Introduction

$\S 1$. Classical Statement of the Results

$\S 1.1$. The Radon-Penrose transform

$\S 1.2$. Action of $S U(p+1, n-p)$

$\S 1.3$. Action of $S L(n+1, \mathbf{R})$

$\S 1.4$. Action of $S L(n+1, \mathbf{C})_{\mathbf{R}}$

$\S 1.5$. Action of $S L((n+1) / 2, \mathbf{H})$

Communicated by M. Kashiwara, May 24, 1999.

1991 Mathematics Subject Classification(s): 35C15, 32L25, 44A12.

*Institut de Mathématiques, 175, rue du Chevaleret, 75013 Paris, France.

e-mail: dagnolo@math.jussieu.fr

** Dip. Mat. Pura ed Appl., Università di Padova, via Belzoni 7, 35131 Padova, Italy. e-mail: maraston@math.unipd.it 
$\S 2 . \quad$ Adjunction Formula for the Radon Transform

$\S 2.1$. Function spaces

$\S 2.2$. Review on integral transforms

$\S 2.3$. Massless field equations

$\S 2.4$. Radon adjunction formula

$\S 3 . \quad$ Proofs and Further Results

$\S 3.1$. The Radon-Penrose transform

§3.2. Action of $S U(p+1, n-p)$

$\S 3.3 . \quad$ Action of $S L(n+1, \mathbf{R})$

$\S 3.4$. Action of $S L(n+1, \mathbf{C})_{\mathbf{R}}$

$\S 3.5$. Action of $S L((n+1) / 2, \mathbf{H})$

$\S 3.6$. More on $S L(n+1, \mathbf{R})$

$\S 3.7$. More on $S L(n+1, \mathbb{C})_{\mathbf{R}}$

$\S A$. Review on Projective Duality

$\S A .1 . \quad$ Adjunction formula

$\S$ A.2. Action of $S L(n+1, \mathbf{R})$

$\S$ A.3. Action of $S U(q+1, n-q)$

References

\section{Introduction}

Let $\mathbb{V}$ be an $(n+1)$-dimensional complex vector space, denote by $\mathbb{P}$ the projective space of lines in $\mathbb{V}$, by $\mathbb{G}=\operatorname{Gr}(p+1 ; \mathbb{V})$ the Grassmann manifold of $(p+1)$-dimensional subspaces, and by $\mathbb{F} \subset \mathbb{P} \times \mathbb{G}$ the incidence relation. The generalized Radon-Penrose transform is associated with the double fibration

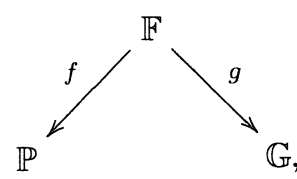

where $f$ and $g$ are induced by the natural projections. This is the graph of the correspondence $\zeta \multimap \widehat{\zeta}=f\left(g^{-1}(\zeta)\right)$, mapping $\zeta \in \mathbb{G}$ to the $p$-dimensional plane of $\mathbb{P}$ that it represents. For different choices of "functions" $\varphi$, the various instances of the Radon $p$-plane transform can thus be expressed as

$$
\varphi \mapsto \int_{g} f^{*} \varphi
$$

Let us recall some examples for $n=3$ and $p=1$ (see $[10,28,12,14,15]$ ).

The Grassmann manifold $\mathbb{G}=\operatorname{Gr}\left(2 ; \mathbb{C}^{4}\right)$ is a complex compact manifold of dimension 4. A point in $\mathbb{G}$ corresponds to a 2-dimensional plane in $\mathbb{C}^{4}$. If 
$A$ is a $4 \times 2$ matrix of rank 2 with complex coefficients, we denote by $[A] \in \mathbb{G}$ the span of its column vectors. An affine chart in $\mathbb{G}$ is obtained by considering the family of 2-dimensional planes $\zeta$ transversal to a fixed 2-dimensional plane in $\mathbf{C}^{4}$. For example, denoting by $I_{2}$ the $2 \times 2$ unit matrix,

$$
\zeta \pitchfork\left[\begin{array}{c}
I_{2} \\
0
\end{array}\right] \quad \Longleftrightarrow \quad \zeta=\left[\begin{array}{l}
Z \\
I_{2}
\end{array}\right] \text { for } Z=\left(\begin{array}{ll}
z_{1}^{1} & z_{1}^{2} \\
z_{2}^{1} & z_{2}^{2}
\end{array}\right), z_{i}^{j} \in \mathbf{C} .
$$

According to $[10], \mathbb{G}$ is a complexification of the conformal compactification of the linear Minkowski space. In particular, Maxwell wave equation (as well as the whole family of massless field equations) extends as a differential operator $\square$ acting on a holomorphic line bundle on $\mathbb{G}$. Using the above system $Z$ of affine coordinates, one has

$$
\square=\partial_{z_{1}^{1}} \partial_{z_{2}^{2}}-\partial_{z_{1}^{2}} \partial_{z_{2}^{1}}
$$

The Grassmann manifold $\mathbb{G}$ is homogeneous for the complex Lie group $S L(4, \mathbf{C})$. In the following table we list some of the real forms of $S L(4, \mathrm{C})$, the associated closed orbit in $\mathbb{G}$, the system $\mathbf{R}^{4} \ni\left(x_{1}, \ldots, x_{4}\right) \mapsto Z$ of affine coordinates in the intersection of the closed orbit with the above affine chart, and the expression of $\square$.

\begin{tabular}{c||c|c|c|}
\hline real form of $S L(4, \mathbf{C})$ & $S U(2,2)$ & $S L(4, \mathbf{R})$ & $S L(2, \mathbf{H})$ \\
\hline closed orbit in $\mathbb{G}$ & $\mathrm{G} \simeq S^{1} \times S^{3}$ & $\mathrm{G} \simeq \mathrm{Gr}\left(2 ; \mathbf{R}^{4}\right)$ & $\mathbf{H} \mathbb{G} \simeq S^{4}$ \\
\hline affine coordinates $Z$ & $\left(\begin{array}{c}x_{1}+x_{2} \\
\frac{x_{3}+\imath x_{4}}{x_{3}+\imath x_{4}} x_{1}-x_{2}\end{array}\right)$ & $\left(\begin{array}{c}x_{1} x_{2} \\
x_{3} x_{4}\end{array}\right)$ & $\left(\begin{array}{c}x_{1}+\imath x_{2} \\
-\left(\overline{x_{3}+\imath x_{4}}\right) \frac{x_{3}+\imath x_{4}}{x_{1}+\imath x_{2}}\end{array}\right)$ \\
\hline expression of $\square$ & $\partial_{x_{1}}^{2}-\partial_{x_{2}}^{2}-\partial_{x_{3}}^{2}-\partial_{x_{4}}^{2}$ & $\partial_{x_{1}} \partial_{x_{4}}-\partial_{x_{2}} \partial_{x_{3}}$ & $\partial_{x_{1}}^{2}+\partial_{x_{2}}^{2}+\partial_{x_{3}}^{2}+\partial_{x_{4}}^{2}$ \\
\hline
\end{tabular}

(a) The Penrose correspondence describes the set of holomorphic solutions of $\square$ on suitable open subsets $U \subset \mathbb{G}$ by the transform (2). In this case, $\varphi \in H^{1}\left(\widehat{U} ; \mathcal{O}_{\mathbb{P}}(-2)\right)$ is a cohomology class of a holomorphic line bundle, and the integral has to be understood as a Leray-Grothendieck residue.

(b) The manifold $\mathrm{G}$ is a conformal compactification of the linear Minkowski space. Denote by $\mathrm{P}$ the closed orbit of $S U(2,2)$ on $\mathbb{P}$. The set of hyperfunctions solutions on $\mathrm{G}$ of the hyperbolic wave equation $\left.\square\right|_{G}$ is obtained by (2), taking for $\varphi \in H_{\mathrm{P}}^{2}\left(\mathbb{P} ; \mathcal{O}_{\mathbb{P}(-2))}\right.$ a cohomology class with support on P.

(c) The real projective space $\mathrm{P}$ is the closed orbit of $S L(4 ; \mathbf{R})$ on $\mathbb{P}$. Let $\varphi \in \mathcal{S}\left(\mathbf{R}^{4}\right)$ be a rapidly decreasing function in an affine chart $\mathbf{R}^{4} \subset \mathrm{P}$. Then (2) represents the usual Radon transform, mapping $\varphi$ to its integrals along the family of 2-dimensional planes of $\mathbf{R}^{4}$. Functions in its image satisfy the ultrahyperbolic John equation $\left.\square\right|_{G}$. 
(d) The elliptic Laplacian $\left.\square\right|_{\mathbf{H G}}$ has no global solutions on the 1-dimensional quaternionic projective space $\mathbb{H} \mathbb{G} \simeq \operatorname{Gr}\left(1 ; \mathbb{H}^{2}\right)$. By (2), this corresponds to the fact that $H^{j}\left(\mathbb{P} ; \mathcal{O}_{\mathbb{P}}(-2)\right)=0$ for any $j$. (Note that the quaternionic group $S L(2, \mathbb{H})$ acts transitively on $\mathbb{P}$.)

Heuristically, the above results appear as different aspects of the complex Radon $p$-plane transform (1), for different choices of geometric configurations in $\mathbb{G}$. The theory of integral transforms for sheaves and $\mathcal{D}$-modules (see $[6,7,21]$, and [4] for an exposition) gives to such vague statement a precise meaning. Let us briefly recall this framework. The analogues of (2) in terms of sheaves and $\mathcal{D}$-modules are the functors

$$
\mathcal{G} \mapsto \mathbb{C}_{\mathbb{F}} \circ \mathcal{G}=R f_{!}\left(g^{-1} \mathcal{G}\right), \quad \text { and } \quad \mathfrak{M} \mapsto \mathfrak{M}^{D} \mathcal{B}_{\mathbb{F}}=D g_{!}\left(D f^{*} \mathfrak{M}\right),
$$

where $g^{-1}, R f_{\text {! }}$ and $D f^{*}, D g_{\text {! }}$ denote the operations of inverse and proper direct image in the derived categories of sheaves and $\mathcal{D}$-modules, respectively. If $\mathcal{G}$ is $\mathbb{R}$-constructible, one associates with it four function spaces on $\mathbb{G}$, that we denote by $\mathcal{C}^{\omega}(\mathcal{G}), \mathcal{C}^{\infty}(\mathcal{G}), \mathcal{C}^{-\infty}(\mathcal{G})$, and $\mathcal{C}^{-\omega}(\mathcal{G})$. For example, if $\mathcal{G}=\mathbb{C}_{G}$ is the constant sheaf along a real analytic manifold $G$, of which $\mathbb{G}$ is a complexification, these are the sheaves of analytic functions, $C^{\infty}$-functions, distributions and hyperfunctions, respectively. A general adjunction formula asserts that, under mild hypotheses, solutions of $\mathfrak{M}$ with values in $\mathcal{C}^{\natural}\left(\mathbb{C}_{\mathbb{F}} \circ \mathcal{G}\right)$ are isomorphic to solutions of $\mathfrak{M}^{D} \circ \mathcal{B}_{\mathbb{F}}$ with values in $\mathcal{C}^{\natural}(\mathcal{G})$, for $\downarrow= \pm \infty$, $\pm \omega$. More precisely,

$$
\operatorname{Sol}\left(\mathfrak{M}, \mathcal{C}^{\natural}\left(\mathbb{C}_{\mathbb{F}} \circ \mathcal{G}\right)\right)[\operatorname{dim} \mathbb{F}-\operatorname{dim} \mathbb{G}] \stackrel{\sim}{\longrightarrow} \operatorname{Sol}\left(\mathfrak{M}^{\circ} \circ \mathcal{B}_{\mathbb{F}}, \mathcal{C}^{\natural}(\mathcal{G})\right) \text {. }
$$

According to [6], the results in [10] imply that massless field equations are represented by $\mathfrak{M} \stackrel{D}{\circ} \mathcal{B}_{\mathbb{F}}$, where $\mathfrak{M}=\mathcal{D}_{\mathbb{P}}(\ell)$ is the $\mathcal{D}_{\mathbb{P}}$-module induced by a line bundle on $\mathbb{P}$. A similar result holds in the general case $n \geq 3,1 \leq p \leq n-1$ (see $[7,8,2,5])$.

Several results on the Radon $p$-plane transform are then obtained from the adjunction formula (3), for different choices of $\mathcal{G}$. For example, the higher dimensional analogue of the Penrose transform (a) corresponds to $\mathcal{G}=\mathbb{C}_{U}$, the constant sheaf on $U \subset \mathbb{G}$. Similarly, the higher dimensional analogues of (b), (d), and of a compactified version of (c), correspond to locally constant sheaves along the closed orbit of one of the real forms of $S L(\mathbb{V})$. Some of these cases have already been considered in [6] for $p=1$ and $n=3$, and in $[7,3]$ for $p=n-1$. The aim of this paper is to investigate systematically the results one gets from (3) for such choices of $\mathcal{G}$, as well as some other related choices. This consists essentially in computing the sheaf-theoretical transforms $\mathbb{C}_{\mathbb{F}} \circ \mathcal{G}$. For a locally closed subset $G \subset \mathbb{G}$, the complex $\mathbf{C}_{\mathbb{F}} \circ \mathbf{C}_{G}$ describes the homology of the family of slices $\mathbb{P} \ni z \multimap \widehat{z} \cap G$. In other words, it is just by using some relatively simple topological arguments that we will be able to recover and generalize the above results on range characterization for the projective, conformal and affine versions of the real Radon transform. 
This paper is divided into three parts. In the first part we state most of the results using a classical formalism, i.e. avoiding the language of derived categories and $\mathcal{D}$-modules. In the second part we recall some results from the theory of integral transforms for sheaves and $\mathcal{D}$-modules, as the adjunction formula (3). Since our statements deal with the first non-vanishing cohomology group, we also give some technical results that allow one to weaken some hypotheses. In the last section we give proofs.

\section{$\S 1$. Classical Statement of the Results}

In this section we state most of our results using a classical formalism, i.e., avoiding the language of derived categories and $\mathcal{D}$-modules. This should be considered as a motivation for the reader not acquainted with such theories. Proofs of the statements, along with some generalizations, are given in Section 3.

\section{§1.1. The Radon-Penrose transform}

For $n \geq 3,1 \leq p \leq n-2$, consider

$$
\begin{cases}\mathbb{V} & \text { a complex vector space of dimension } n+1, \\ \mathbb{P}=\operatorname{Gr}(1 ; \mathbb{V}) & \text { a complex projective space of dimension } n, \\ \mathbb{G}=\operatorname{Gr}(p+1 ; \mathbb{V}) & \text { the family of projective } p \text {-dimensional planes } \\ \mathbb{F}=\{(z, \zeta) \in \mathbb{P} \times \mathbb{G}: z \in \zeta\} & \text { in } \mathbb{P}, \\ & \text { the incidence relation, }\end{cases}
$$

where $\operatorname{Gr}(q ; \mathbb{V})$ denotes the Grassmann manifold of $q$-dimensional subspaces in $\mathbb{V}$. Recall that $\mathbb{G}$ is a complex compact manifold of dimension $(p+1)(n-p)$, that the flag manifold $\mathbb{F}$ has dimension $n+p(n-p)$, and that $\mathbb{P}, \mathbb{G}$ and $\mathbb{F}$ are homogeneous with respect to the Lie group $S L(\mathbb{V}) \simeq S L(n+1, \mathbf{C})$. (For the extreme case $p=n-1$, refer to the Appendix.)

The geometric background of the generalized Radon-Penrose transform is given by the double fibration

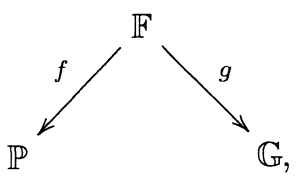

where $f$ and $g$ are the natural projections (see $[10,28,12,14,15])$. Given a plane $\zeta \in \mathbb{G}$, one denotes by $\widehat{\zeta}=f\left(g^{-1}(\zeta)\right) \subset \mathbb{P}$ the set of its points. Similarly, for $U \subset \mathbb{G}$ and $z \in \mathbb{P}$ one sets $\widehat{U}=f\left(g^{-1}(U)\right) \subset \mathbb{P}$ and $\widehat{z}=g\left(f^{-1}(z)\right) \subset \mathbb{G}$. 
As we recalled in the Introduction, for $p=1$ and $n=3,[10]$ identifies the manifold $\mathbb{G}$ with a conformal compactification of the complexified Minkowski space. On the Minkowski space lives the family of massless field equations. Since such equations are conformally invariant, they extend as a family of differential operators acting on holomorphic sections of homogeneous vector bundles over $\mathbb{G}$. For $n \geq 3,0 \leq p \leq n-2$, we denote by

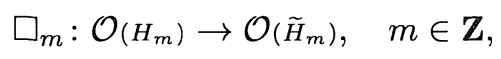

their higher dimensional analogue, and we refer to Section 2.3 for details. In our notations, the operator $\square_{m}$ on Minkowski space is the massless field equation of helicity $-(m+1) / 2$, so that $\square_{-1}$ is Maxwell's wave equation.

In this paper, for $m \in \mathbf{Z}$ we set

$$
m^{*}=-m-p-2 .
$$

Note that for $p=0$ and $m^{*}<0$ one has $\mathbb{G}=\mathbb{P}, \square_{m^{*}}=0$ (i.e. no differential equations appear), and

$$
\mathcal{O}\left(H_{m^{*}}\right)=\mathcal{O}_{\mathbb{P}}\left(m^{*}\right)
$$

where $\mathcal{O}_{\mathbb{P}}\left(m^{*}\right)$ is the usual notation for $-m^{*}$-th tensor power of the tautological line bundle.

One says that an open subset $U \subset \mathbb{G}$ is $\mathbb{F}$-elementary if for any $z \in \widehat{U}$ the set $\widehat{z} \cap U$ is connected, and its homology groups with complex coefficients vanish in degree $1, \ldots, p$.

Proposition 1.1.1. Let $m \in \mathbb{Z}$, with $m^{*}<0$, and let $U$ be an $\mathbb{F}$ elementary open subset of $\mathbb{G}$. Then, one has an isomorphism

$$
H^{p}\left(\widehat{U} ; \mathcal{O}_{\mathbb{P}}\left(m^{*}\right)\right) \stackrel{\sim}{\longrightarrow}\left\{\psi \in \Gamma\left(U ; \mathcal{O}_{\mathbb{G}}\left(H_{m}\right)\right): \square_{m} \psi=0\right\},
$$

the lower cohomology groups being zero.

(A proof is given in Section 3.1.)

This result was obtained in [10] for $n=3$ and $p=1$, and is discussed in [2] in an equivariant framework.

Example 1.1.2. The case $p+1=n-p$ is of particular interest in representation theory (see e.g. [26]). There, one takes for $U$ the set of $\zeta \in \mathbb{G}$ such that $\left.\omega\right|_{\zeta}$ is positive definite, where $\omega$ is a nondegenerate hermitian form on $\mathbb{V}$ of signature $(p+1, p+1)$. Since $\widehat{z} \cap U$ is contractible for $z \in \widehat{U}$, the above result applies. (See also Section A.3 for the extreme case $p=n-1$.)

Example 1.1.3. Recall that an open subset $D \subset \mathbb{P}$ is said to be $p$ linearly concave if through any $z \in D$ there passes a projective $p$-dimensional 
plane contained in $D$. In this case, setting $U=\mathbb{G} \backslash(\widehat{\mathbb{P} \backslash D})=\{\zeta \in \mathbb{G}: \widehat{\zeta} \subset D\}$, one has $D=\widehat{U}$. For example, take $D=\mathbb{P} \backslash \mathbb{L}$, where $\mathbb{L} \subset \mathbb{P}$ is a projective plane of dimension $n-p-1$. Then, $U$ is an affine chart in $\mathbb{G}$, and the above proposition applies. Note that since $U$ is determined by a subset of $\mathbb{P}$, the hypotheses could be weakened (see $[13 ;,[16]$ ).

\section{§1.2. Action of $S U(p+1, n-p)$}

\subsubsection{Conformal case.}

Let $\omega$ be a nondegenerate hermitian form on $\mathbb{V}$ of signature $(p+1, n-p)$, and let us assume that $p+1 \leq n-p$. The Lie group $S U(\omega ; \mathbb{V}) \simeq S U(p+1, n-p)$ is a real form of $S L(\mathbb{V})$, and hence acts on $\mathbb{P}$ and $\mathbb{G}$. The closed orbits of $S U(\omega ; \mathbb{V})$ in $\mathbb{P}$ and $\mathbb{G}$ are, respectively,

$$
\left\{\begin{array}{l}
\mathrm{P} \quad \text { the set of } \omega \text {-isotropic lines in } \mathbb{V}, \\
\mathrm{G} \text { the set } \omega \text {-isotropic }(p+1) \text {-dimensional planes in } \mathbb{V}
\end{array}\right.
$$

where a subspace $\zeta \subset \mathbb{V}$ is called $\omega$-isotropic if $\left.\omega\right|_{\zeta}=0$.

Recall that a real submanifold $M$ of a complex manifold $X$ is called generic if $T M+{ }_{M} i T M=\left.T X\right|_{M}$, where $T M$ denotes the tangent bundle to $M$. Note that orbits of real forms of a simple complex Lie group acting on a homogeneous manifold are always generic. Concerning (1.2.1), $\mathrm{P}$ is a real hypersurface of $\mathbb{P}$ with non-degenerate Levi form of signature $(p, n-p-1)$, and $\mathrm{G}$ is a generic submanifold of $\mathbb{G}$ of codimension $(p+1)^{2}$. We denote by

$$
\mathcal{A}_{\mathrm{G}}^{\bar{\partial}^{b}}=\left.\mathcal{O}_{\mathbb{G}}\right|_{\mathrm{G}}, \quad \mathcal{B}_{\mathrm{G}}^{\bar{\partial}^{b}}=H_{\mathrm{G}}^{(p+1)^{2}} \mathcal{O}_{\mathbb{G}},
$$

the analytic and hyperfunction CR-functions on $\mathrm{G}$ (i.e., solutions of the tangential Cauchy-Riemann system $\bar{\partial}^{b}$ ). If $\mathcal{G}$ is an $\mathcal{O}_{\mathbb{G}}$-module, we shall use the short-hand notation

$$
\mathcal{G}\left(H_{m}\right)=\mathcal{G} \otimes_{\mathcal{O}_{\mathbb{G}}} \mathcal{O}\left(H_{m}\right)
$$

Proposition 1.2.1. For $m^{*}<0$ one has a commutative diagram

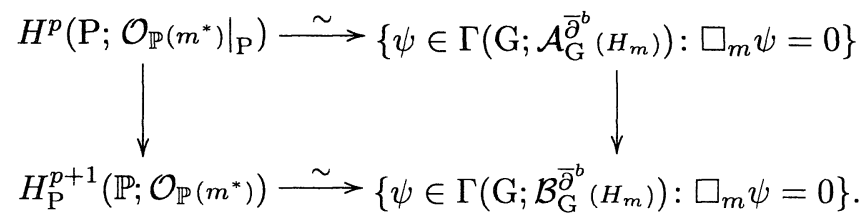

(A proof is given in Section 3.2.1.)

When $p+1=n-p, \mathbb{G}$ is a complexification of $\mathrm{G}$, and hence $\mathcal{A}_{\mathrm{G}}^{\bar{\partial}^{b}}=\mathcal{A}_{\mathrm{G}}$ and $\mathcal{B}_{\mathrm{G}}^{\bar{\partial}^{b}}=\mathcal{B}_{\mathrm{G}}$ are the sheaves of analytic functions and hyperfunctions, respectively. 
For $n=3$ and $p=1$ the manifold $\mathrm{G}$ is the conformal compactification of the Minkowski space $\mathbb{R}^{4}$, and the above result is due to [28].

\subsubsection{Conformal affine case.}

Let $\zeta_{\circ} \in \mathrm{G}$, and denote by $\zeta_{\circ}^{\perp}$ its $\omega$-orthogonal, which is an $(n-p)$ dimensional subspace of $\mathbb{V}$ containing $\zeta_{0}$. The "light cone" with vertex $\zeta_{0}$ is the subset of $\mathrm{G}$ described by those $\zeta \in \mathrm{G}$ having non-transversal intersection with $\zeta_{\circ}^{\perp}$. Consider

$$
\begin{cases}\mathrm{P}_{\circ}=\mathrm{P} \cap \mathbb{P}_{\circ}, & \mathbb{P}_{\circ}=\left\{z \in \mathbb{P}: z \cap \zeta_{\circ}^{\perp}=0\right\} \\ \mathrm{G}_{\circ}=\mathrm{G} \cap \mathbb{G}_{\circ}, & \mathbb{G}_{\circ}=\left\{\zeta \in \mathbb{G}: \zeta \cap \zeta_{\circ}^{\perp}=0\right\}\end{cases}
$$

Note that $G_{\circ}$ and $\mathbb{G}_{\circ}$ are affine charts in $G$ and $\mathbb{G}$, respectively, obtained by removing the light cone at infinity.

Proposition 1.2.2. For $m^{*}<0$ one has

$$
H_{\mathrm{P}_{\circ}}^{p+1}\left(\mathbb{P}_{\circ} ; \mathcal{O}_{\mathbb{P}}\left(m^{*}\right)\right) \stackrel{\sim}{\longrightarrow}\left\{\psi \in \Gamma\left(\mathrm{G}_{\circ} ; \mathcal{B}_{\mathrm{G}}^{\bar{\partial}^{b}}\left(H_{m}\right)\right): \square_{m} \psi=0\right\} .
$$

Moreover, if $p+1 \neq n-p-1$, the natural restriction map

$$
\left\{\psi \in \Gamma\left(\mathrm{G} ; \mathcal{B}_{\mathrm{G}}^{\bar{\partial}^{b}}\left(H_{m}\right)\right): \square_{m} \psi=0\right\} \rightarrow\left\{\psi \in \Gamma\left(\mathrm{G}_{\circ} ; \mathcal{B}_{\mathrm{G}}^{\bar{\partial}^{b}}\left(H_{m}\right)\right): \square_{m} \psi=0\right\}
$$

is surjective.

(A proof is given in Section 3.2.2.)

For $n=3$ and $p=1$ this is a result due to [1]. In this case, $\mathrm{G}_{\circ} \simeq \mathbf{R}^{4}$ is the Minkowski space, and the second part of the statement above asserts that the light cone at infinity is a removable singularity for hyperfunction solutions of the massless field equations.

\section{$\S 1.3 . \quad$ Action of $S L(n+1, \mathbf{R})$}

\subsubsection{Real projective case。}

Let $\mathrm{V}$ be a real vector space of dimension $n+1$, of which $\mathbb{V} \simeq \mathbf{C} \otimes_{\mathbf{R}} \mathrm{V}$ is a complexification. The Lie group $S L(\mathrm{~V}) \simeq S L(n+1, \mathbf{R})$ is a real form of $S L(\mathbb{V})$. Its closed orbits on $\mathbb{P}$ and $\mathbb{G}$ are identified with, respectively,

$$
\begin{cases}\mathrm{P}=\mathrm{Gr}(1 ; \mathrm{V}) & \text { a real projective space of dimension } n \\ \mathrm{G}=\mathrm{Gr}(p+1 ; \mathrm{V}) & \text { the family of projective } p \text {-dimensional planes in } \mathrm{P}\end{cases}
$$

which are totally real submanifolds.

Let $\Omega$ be the open subset of $\mathrm{V}^{p+1}$ consisting of $(p+1)$-uples of linearly independent vectors, and denote by $q: \Omega \rightarrow \mathrm{G}$ the projection mapping $\left(v_{1}, \ldots, v_{p+1}\right)$ 
to the subspace of $\mathrm{V}$ that they generate. For $\varepsilon \in \mathbf{Z} / 2 \mathbf{Z}$, consider the real line bundle over $\mathrm{G}$ whose sheaf of $C^{\infty}$ sections, that we denote by $\mathcal{C}_{\mathrm{G}}^{\infty}(0 \mid \varepsilon)$, is the subsheaf of $q_{*} \mathcal{C}_{\Omega}^{\infty}$ described by the homogeneity condition

$$
\psi(\mu \xi)=(\operatorname{sgn} \operatorname{det} \mu)^{\varepsilon} \psi(\xi) \quad \text { for } \mu \in G L(p+1 ; \mathbf{R}) .
$$

Moreover, for $m \in \mathbf{Z}$ set

$$
\mathcal{C}_{\mathrm{G}}^{\infty}\left(H_{m} \mid \varepsilon\right)=\mathcal{C}_{\mathrm{G}}^{\infty}(0 \mid \varepsilon) \otimes_{\mathcal{C}_{\mathrm{G}}^{\infty}} \mathcal{C}^{\infty}\left(H_{m}\right)
$$

In particular, for $m^{*}<0$ the sheaf $\mathcal{C}_{\mathrm{P}}^{\infty}\left(H_{m^{*}} \mid \varepsilon\right)=\mathcal{C}_{\mathrm{P}}^{\infty}\left(m^{*} \mid \varepsilon\right)$ is the subsheaf of $q_{*} \mathcal{C}_{\mathrm{V} \backslash\{0\}}^{\infty}$ whose sections $\varphi$ satisfy

$$
\varphi(\lambda x)=(\operatorname{sgn} \lambda)^{\varepsilon} \lambda^{m^{*}} \varphi(x) \quad \text { for } \lambda \in \mathbf{R}^{\times} .
$$

Proposition 1.3.1. For $m^{*}<0$ one has the isomorphism

$$
\Gamma\left(\mathrm{P} ; \mathcal{C}_{\mathrm{P}}^{\infty}\left(m^{*} \mid-p-1\right)\right) \stackrel{\sim}{\longrightarrow}\left\{\psi \in \Gamma\left(\mathrm{G} ; \mathcal{C}_{\mathrm{G}}^{\infty}\left(H_{m} \mid-1\right)\right): \square_{m} \psi=0\right\},
$$

and the similar isomorphisms obtained when $\mathcal{C}^{\infty}$ is replaced by real analytic functions, distributions, or hyperfunctions.

(A proof is given in Section 3.3.1.)

This result appears for example in [12, p. 91-92] for the $C^{\infty}$ case. For $m=-1, m^{*}=-p-1$, the above morphism is a projective compactification of the classical Radon $p$-plane transform, where functions on $\mathrm{P}$ are integrated along $p$-dimensional planes.

\subsubsection{Real affine case.}

Let us consider the setting of the affine Radon transform

$$
\begin{cases}A=P \backslash H & \text { for a hyperplane } H \subset P, \\ G_{A} \subset G & \text { the set of } \xi \in G \text { with } \widehat{\xi} \not \subset H .\end{cases}
$$

The Schwartz space $\mathcal{S}(\mathrm{A})$ of rapidly decreasing $C^{\infty}$-functions on the affine space $A \simeq \mathbf{R}^{n}$, is identified with the subspace of $\Gamma\left(P ; \mathcal{C}_{\mathrm{P}}^{\infty}\left(m^{*} \mid-p-1\right)\right)$ whose sections vanish up to infinite order on $H$.

The map $q: \xi \mapsto \xi \cap \mathrm{H}$ endows $\mathrm{G}_{\mathrm{A}}$ with a structure of $(n-p)$-dimensional real vector bundle over the compact Grassmann manifold of $(p-1)$-dimensional projective planes in $\mathrm{H}$. Let us denote by $\mathcal{S}_{\left(H_{m} \mid-1\right)}\left(\mathrm{G}_{\mathrm{A}}\right)$ the subspace of $\Gamma\left(\mathrm{G}_{\mathrm{A}} ; \mathcal{C}_{\mathrm{G}}^{\infty}\left(H_{m} \mid-1\right)\right)$ whose sections are rapidly decreasing along the fibers of $q$. As above, this is identified with the subspace of $\Gamma\left(\mathrm{G} ; \mathcal{C}_{\mathrm{G}}^{\infty}\left(H_{m} \mid-1\right)\right)$ whose sections vanish up to infinite order on $G \backslash G_{A}$. 
Proposition 1.3.2. For $m^{*}<0$, the isomorphism of Proposition 1.3.1 induces an isomorphism

$$
\mathcal{S}(\mathrm{A}) \stackrel{\sim}{\longrightarrow}\left\{\psi \in \mathcal{S}_{\left(H_{m} \mid-1\right)}\left(\mathrm{G}_{\mathrm{A}}\right): \square_{m} \psi=0\right\} .
$$

(A proof is given in Section 3.3.2.)

For $m=-1$, this result appears, for example, in [12, p. 85]. Recall that for $p=1$ and $n=3$, the operator $\left.\square_{-1}\right|_{\mathrm{G}_{\mathrm{A}}}$ is the ultrahyperbolic operator of John [17].

\section{§1.4. Action of $S L(n+1, \mathbf{C})_{\mathbf{R}}$}

As we shall see in Section 3.3.1, the topology underlying Proposition 1.3.1 is quite intricate. A somewhat simpler situation is obtained by considering the real analytic manifold underlying the complex Grassmann manifold.

\subsubsection{Realified complex projective case.}

Denote by $\overline{\mathbb{P}}$ the manifold $\mathbb{P}$ endowed with the conjugate complex analytic structure (i.e. the ringed space $\left(\mathbb{P}, \overline{\mathcal{O}_{\mathbb{P}}}\right)$, where $\overline{\mathcal{O}_{\mathbb{P}}}$ is the sheaf of antiholomorphic functions). The diagonal $\mathbb{P}_{\mathbf{R}} \subset \mathbb{P} \times \overline{\mathbb{P}}$ is identified with the space $\mathbb{P}$, endowed with its underlying structure of real analytic manifold. We thus consider

$$
\begin{cases}\mathbb{P}_{\mathbf{R}} \subset \mathbb{P} \times \overline{\mathbb{P}} & \text { the realified complex projective space, } \\ \mathbb{G}_{\mathbf{R}} \subset \mathbb{G} \times \overline{\mathbb{G}} & \text { the realified complex Grassmann manifold }\end{cases}
$$

Note that $\mathbb{P}_{\mathbf{R}} \subset \mathbb{P} \times \overline{\mathbb{P}}$ is the closed orbit of the real form $S L(\mathbb{V})_{\mathbf{R}}$ of the complex Lie group $S L(\mathbb{V}) \times S L(\overline{\mathbb{V}})$, and $\mathbb{G}_{\mathbf{R}}$ its closed orbit in $\mathbb{G} \times \overline{\mathbb{G}}$.

For $m, \widetilde{m} \in \mathbb{Z}$, denote by $\mathcal{C}_{\mathbb{G}_{\mathbf{R}}}^{\infty}\left(H_{m}, \bar{H}_{\bar{m}}\right)$ the sheaf of $C^{\infty}$ sections of $\left(H_{m} \otimes\right.$ $\left.\bar{H}_{\tilde{m}}\right)\left.\right|_{\mathbb{G}_{\mathbf{R}}}$. Note in particular that, for $m^{*}<0$, sections $\varphi \in \mathcal{C}_{\mathbb{P}_{\mathbf{R}}}^{\infty}\left(H_{m^{*}}, \bar{H}_{m^{*}}\right) \simeq$ $\mathcal{C}_{\mathbb{P}_{\mathbf{R}}}^{\infty}\left(m^{*}, m^{*}\right)$ satisfy the homogeneity condition

$$
\varphi(\lambda z)=|\lambda|^{2 m^{*}} \varphi(z) \quad \text { for } \lambda \in \mathbf{C}^{\times} .
$$

Proposition 1.4.1. For $m^{*}, \widetilde{m}^{*}<0$ one has an isomorphism

$$
\Gamma\left(\mathbb{P}_{\mathbf{R}} ; \mathcal{C}_{\mathbb{P}_{\mathbf{R}}}^{\infty}\left(m^{*}, \tilde{m}^{*}\right)\right) \stackrel{\sim}{\rightarrow}\left\{\psi \in \Gamma\left(\mathbb{G}_{\mathbf{R}} ; \mathcal{C}_{\mathbb{G}_{\mathbf{R}}}^{\infty}\left(H_{m}, \bar{H}_{\tilde{m}}\right)\right): \square_{m} \psi=\bar{\square}_{\widetilde{m}} \psi=0\right\},
$$

and the similar isomorphisms obtained when $\mathcal{C}^{\infty}$ is replaced by real analytic functions, distributions, or hyperfunctions.

(A proof is given in Section 3.4.1.)

In the $C^{\infty}$ case, and for $m=\widetilde{m}$, this result is proven in [12]. 


\subsubsection{Realified complex affine case.}

Let us consider the affine setting

$$
\begin{cases}\mathbb{A}=\mathbb{P} \backslash \mathbb{H} & \text { for a hyperplane } \mathbb{H} \subset \mathbb{P} \\ \mathbb{G}_{\mathbb{A}} \subset \mathbb{G} & \text { the set of } \zeta \in \mathbb{G} \text { with } \widehat{\zeta} \not \subset \mathbb{H} .\end{cases}
$$

With notations similar to those in Proposition 1.3.2, one has

Proposition 1.4.2. For $m^{*}, \widetilde{m}^{*}<0$, there is an isomorphism

$$
\mathcal{S}\left(\mathbb{A}_{\mathbf{R}}\right) \stackrel{\sim}{\longrightarrow}\left\{\psi \in \mathcal{S}_{\left(H_{m}, \bar{H}_{\widetilde{m}}\right)}\left(\mathbb{G}_{\mathbb{A}_{\mathbf{R}}}\right): \square_{m} \psi=\bar{\square}_{\widetilde{m}} \psi=0\right\} .
$$

(A proof is given in Section 3.4.2.)

For $m=\widetilde{m}=-1$, this result appears in [12].

\section{§1.5. Action of $S L((n+1) / 2, \mathbf{H})$}

Assume that $n$ and $p$ are odd, and let $\mathbb{W}$ be a complex vector space of dimension $(n+1) / 2$, such that $\mathbb{V}=\mathbb{W} \oplus \mathbb{W}$. Denote by $\mathbf{H}$ the skew field of quaternions, which is a division algebra over $\mathbf{R}$ with basis $1, i, j, k$ satisfying $i^{2}=$ $j^{2}=k^{2}=-1, i j=k, j k=i, k i=j$. The identification $\mathbf{C} \oplus \mathbf{C} \stackrel{\sim}{\longrightarrow} \mathbf{C} \oplus j \mathbf{C}=\mathbf{H}$ induces an isomorphism $h: \mathbb{V} \stackrel{\sim}{\longrightarrow} \mathbb{W} \otimes_{\mathbf{C}} \mathbf{H}$, given by $\left(w_{1}, w_{2}\right) \mapsto w_{1} \otimes 1+\bar{w}_{2} \otimes j$. Since $h$ is right $\mathbf{C}$-linear, the group $S L\left(\mathbb{W} \otimes_{\mathbf{C}} \mathbf{H}\right) \simeq S L((n+1) / 2, \mathbf{H})$ of $\mathbf{H}$-linear automorphisms of the quaternionic vector space $\mathbb{W} \otimes_{\mathbf{C}} \mathbf{H}$ is identified with a real form of $S L(\mathbb{V})$. The action of $S L\left(\mathbb{W} \otimes_{\mathbf{C}} \mathbf{H}\right)$ on $\mathbb{P}$ is transitive, while its closed orbit $\mathbf{H} \mathbb{G}$ in $\mathbb{G}$ is described by the family of complex $(p+1)$-dimensional subspaces $\zeta \subset \mathbb{V}$ such that $h(\zeta)$ is a quaternionic $(p+1) / 2$-dimensional subspace of $\mathbb{W} \otimes_{\mathbf{C}} \mathbf{H}$. Since $\mathbf{H} \mathbb{G}$ is generic, and has half of the dimension of $\mathbb{G}$, it is totally real in $\mathbb{G}$.

Proposition 1.5.1. For $m^{*}<0$ one has

$$
\left\{\psi \in \Gamma\left(\mathbf{H} \mathbb{G} ; \mathcal{B}_{\mathbf{H} \mathbb{G}}\left(H_{m}\right)\right): \square_{m} \psi=0\right\}=0 .
$$

(A proof is given in Section 3.5.)

\section{§2. Adjunction Formula for the Radon Transform}

In the first part of this section, we recall the formalism of integral transforms for sheaves and $\mathcal{D}$-modules, as developed in [6, 7] and [21] (see [4] for an exposition). In the last part, we apply these methods to get a general adjunction formula for the Radon transform, already considered in [6] for $p=1$ and $n=3$, and in [7] for the extreme case $p=n-1$. 


\section{§2.1. Function spaces}

On a complex manifold $X$, denote by $\mathcal{O}_{X}$ its structural sheaf, and by $\mathcal{D}_{X}$ the sheaf of linear differential operators. Let $\mathbb{D}^{\mathrm{b}}\left(\mathbf{C}_{X}\right)$ and $\mathbb{D}^{\mathrm{b}}\left(\mathcal{D}_{X}\right)$ be the bounded derived category of sheaves of $\mathbb{C}$-vector spaces and $\mathcal{D}_{X}$-modules respectively, and consider their full triangulated subcategories $\mathbb{D}_{\mathbb{R}-c}^{b}\left(\mathbb{C}_{X}\right)$ and $\mathbb{D}_{\text {coh }}^{\mathrm{b}}\left(\mathcal{D}_{X}\right)$ of objects with $\mathbb{R}$-constructible and coherent cohomology, respectively.

With $\mathcal{F} \in \mathbb{D}_{\mathbf{R}-\mathbf{c}}^{\mathrm{b}}\left(\mathbb{C}_{X}\right)$ one associates the (complexes of) sheaves

$$
\begin{aligned}
\mathcal{C}^{\omega}(\mathcal{F})=\mathcal{F} \otimes \mathcal{O}_{X}, & \mathcal{C}^{\infty}(\mathcal{F})=\mathcal{F} \stackrel{\text { }}{\otimes} \mathcal{O}_{X}, \\
\mathcal{C}^{-\infty}(\mathcal{F})=\operatorname{THom}\left(\mathcal{F}^{\prime}, \mathcal{O}_{X}\right), & \mathcal{C}^{-\omega}(\mathcal{F})=R \mathcal{H o m}\left(\mathcal{F}^{\prime}, \mathcal{O}_{X}\right),
\end{aligned}
$$

where $\mathcal{F}^{\prime}=R \mathcal{H}$ om $\left(\mathcal{F}, \mathbf{C}_{X}\right)$ denotes the dual of $\mathcal{F}$, and THom $\left(\cdot, \mathcal{O}_{X}\right)$ and $\cdot \stackrel{w}{\otimes} \mathcal{O}_{X}$ are the functors of temperate and formal cohomology introduced in [19] and [21], respectively. For $\mathfrak{M}, \mathcal{C} \in \mathbb{D}^{\mathrm{b}}\left(\mathcal{D}_{X}\right)$, we denote by

$$
\begin{aligned}
\operatorname{Sol}(\mathfrak{M}, \mathcal{C}) & =\operatorname{RHom}_{\mathcal{D}_{X}}(\mathfrak{M}, \mathcal{C}) \\
\operatorname{Sol}(\mathfrak{M}, \mathcal{C}) & =\operatorname{R\Gamma }(X ; \operatorname{Sol}(\mathfrak{M}, \mathcal{C}))
\end{aligned}
$$

the complexes of local and global solutions to $\mathfrak{M}$ with values in $\mathcal{C}$.

In order to discuss some examples, recall that if $A \subset X$ is a locally closed subset, one denotes by $\mathbb{C}_{A \mid X}$ (or $\mathbb{C}_{A}$, if there is no risk of confusion) the sheaf whose restriction to $A$ is the constant sheaf with fiber $\mathbf{C}$ and which is zero on $X \backslash A$.

- If $Y \subset X$ is a complex analytic subset, then $\mathcal{C}^{\infty}\left(\mathbf{C}_{Y}\right)=\mathcal{O}_{X} \widehat{\mid}_{Y}$ is the formal restriction along $Y$, and $\mathcal{C}^{-\infty}\left(\mathbb{C}_{Y}^{\prime}\right)=\mathrm{R} \Gamma_{[Y]} \mathcal{O}_{X}$ is the algebraic cohomology with support on $Y$.

- Let $M$ be a real analytic manifold, of which $X$ is a complexification, and denote by

$$
\mathcal{C}_{M}^{\omega} \subset \mathcal{C}_{M}^{\infty} \subset \mathcal{C}_{M}^{-\infty} \subset \mathcal{C}_{M}^{-\omega}
$$

the sheaves of of real analytic functions, $C^{\infty}$-functions, Schwartz distributions and Sato hyperfunctions, respectively. Then, for $\emptyset= \pm \infty, \pm \omega$,

$$
\mathcal{C}^{\natural}\left(\mathbf{C}_{M \mid X}\right)=\mathcal{C}_{M}^{\natural} .
$$

- If $Z \subset M$ is a closed sub-analytic subset, then

$$
\left\{\begin{array}{l}
\mathcal{C}^{\infty}\left(\mathbf{C}_{(M \backslash Z) \mid X}\right)=\mathcal{I}_{Z, M}^{\infty}, \\
\mathcal{C}^{-\infty}\left(\mathbf{C}_{Z \mid X}\right)[\operatorname{dim} M]=\Gamma_{Z} \mathcal{D} b_{M} \otimes \text { or }_{M},
\end{array}\right.
$$

where $\mathcal{I}_{Z, M}^{\infty}$ denotes the ideal of $\mathcal{C}_{M}^{\infty}$ of functions vanishing up to infinite order on $Z$, and or $_{M}$ the orientation sheaf. 
- Let $N \subset X$ be a real analytic generic submanifold. Then

$$
\mathcal{C}^{\infty}\left(\mathbf{C}_{N \mid X}\right)=\operatorname{Sol}\left(\bar{\partial}^{b}, \mathcal{C}_{N}^{\infty}\right)
$$

is the solution complex to the tangential Cauchy-Riemann system with values in $\mathcal{C}_{N}^{\infty}$, and similarly for $\infty$ replaced by $-\infty, \pm \omega$.

- Let $H \rightarrow X$ be a holomorphic vector bundle. We denote be $\mathcal{O}_{(H)}$ the sheaf of its holomorphic sections, and by $\mathcal{D}(H)=\mathcal{D}_{X} \otimes_{\mathcal{O}_{X}} \mathcal{O}_{(H)}$ the induced locally free left $\mathcal{D}_{X}$-module. Then

$$
\operatorname{Sol}\left(\mathcal{D}_{\left(H^{*}\right)}, \mathcal{O}_{X}\right) \simeq \mathcal{O}(H)
$$

\section{§2.2. Review on integral transforms}

Let $X$ and $Y$ be complex manifolds, and consider the projections

$$
X \underset{q_{1}}{\longleftarrow} X \times Y \underset{q_{2}}{\longrightarrow} Y .
$$

One denotes by

$$
q_{1}^{-1}, \otimes, R q_{2 !}, \quad \text { and } \quad D q_{1}^{*}, \stackrel{D}{\otimes}, D q_{2 !},
$$

the three operations of inverse image, tensor product, and proper direct image, in the categories of sheaves and $\mathcal{D}$-modules, respectively. An expression like

$$
\varphi(x) \mapsto \int \varphi(x) \cdot k(x, \xi),
$$

which represents an integral transform, has a $\mathcal{D}$-module analog in the functor

$$
\mathfrak{M} \mapsto \mathfrak{M} \stackrel{D}{\circ} \mathfrak{K}=D q_{2 !}\left(D q_{1}^{*} \mathfrak{M} \stackrel{D}{\otimes} \mathfrak{K}\right),
$$

where $\mathfrak{M}$ and $\mathfrak{K}$ are (complexes of) $\mathcal{D}$-modules on $X$ and on $X \times Y$, respectively. Similarly, at the level of sheaves, one considers the functor

$$
\mathcal{G} \mapsto \mathcal{K} \circ \mathcal{G}=R q_{1 !}\left(q_{2}^{-1} \mathcal{G} \otimes \mathcal{K}\right)
$$

where $\mathcal{G}$ and $\mathcal{K}$ are (complexes of) sheaves on $Y$ and on $X \times Y$, respectively.

Recall that with $\mathfrak{M} \in \mathbf{D}_{\text {coh }}^{\mathrm{b}}\left(\mathcal{D}_{X}\right)$ one associates its characteristic variety $\operatorname{char}(\mathfrak{M})$ (see [18]), which is a closed conic involutive subset of the cotangent bundle $T^{*} X$. Recall also that the Riemann-Hilbert correspondence establishes an equivalence between the categories $\mathbf{D}_{\mathrm{rh}}^{\mathrm{b}}\left(\mathcal{D}_{X \times Y}\right)$ of regular holonomic modules, and $\mathbf{D}_{\mathbf{C}-\mathrm{c}}^{\mathrm{b}}\left(\mathbf{C}_{X \times Y}\right)$ of $\mathbf{C}$-constructible sheaves, by the assignments

$$
\begin{aligned}
\mathfrak{K} & \longmapsto \operatorname{Sol}\left(\mathfrak{K}, \mathcal{O}_{X \times Y}\right), \\
\operatorname{THom}\left(\mathcal{K}, \mathcal{O}_{X \times Y}\right) & \longleftrightarrow
\end{aligned}
$$


In the following, we will denote by $X$ the zero-section of $T^{*} X$.

Theorem 2.2.1. (see [6,7] and [21]) Let $\mathcal{K} \in \mathbf{D}_{\mathbf{C}-\mathrm{c}}^{\mathrm{b}}\left(\mathbf{C}_{X \times Y}\right)$ and $\mathfrak{K} \in$ $\mathbf{D}_{\mathrm{rh}}^{\mathrm{b}}\left(\mathcal{D}_{X \times Y}\right)$ be interchanged by the Riemann-Hilbert correspondence. Assume that

(a) $q_{1}$ and $q_{2}$ are proper on $\operatorname{supp}(\mathfrak{K})$,

(b) $\operatorname{char}(\mathfrak{K}) \cap\left(T^{*} X \times Y\right) \subset X \times Y$.

Then, for any $\mathfrak{M} \in \mathbb{D}_{\text {coh }}^{\mathrm{b}}\left(\mathcal{D}_{X}\right), \mathcal{G} \in \mathbb{D}_{\mathbf{R}-\mathrm{c}}^{\mathrm{b}}\left(\mathbf{C}_{Y}\right)$, and $\sharp= \pm \infty$, $\pm \omega$, there is an isomorphism

$$
\operatorname{Sol}\left(\mathfrak{M}, \mathcal{C}^{\natural}(\mathcal{K} \circ \mathcal{G})\right)[\operatorname{dim} X] \simeq \operatorname{Sol}\left(\mathfrak{M} \stackrel{D}{\circ} \mathfrak{K}, \mathcal{C}^{\natural}(\mathcal{G})\right)
$$

In particular, let $S \subset X \times Y$ be a closed submanifold, and consider the double fibration

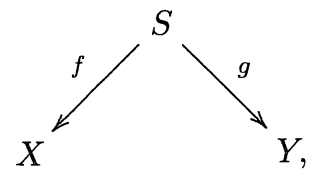

where $f$ and $g$ are the restrictions to $S$ of the projections $q_{1}$ and $q_{2}$. In this case, it is natural to consider the perverse sheaf $\mathcal{K}=\mathbf{C}_{S \mid X \times Y}[-\operatorname{codim} S]$, to which corresponds the regular holonomic module $\mathfrak{K}=\mathcal{B}_{S \mid X \times Y}$ of holomorphic hyperfunctions along $S$. Notice that

$$
\mathrm{C}_{S} \circ \mathcal{G} \simeq R f_{!} g^{-1} \mathcal{G}, \quad \mathfrak{M} \stackrel{D}{\circ} \mathcal{B}_{S} \simeq D g_{!} D f^{*} \mathfrak{M}
$$

Corollary 2.2.2. (see [6] and [21]) Let $S$ be a closed submanifold of $X \times Y$. Assume that $f$ and $g$ are smooth and proper. Then, for any $\mathfrak{M} \in$ $\mathbf{D}_{\text {coh }}^{\mathrm{b}}\left(\mathcal{D}_{X}\right), \mathcal{G} \in \mathbf{D}_{\mathbf{R}-\mathrm{c}}^{\mathrm{b}}\left(\mathbf{C}_{Y}\right)$, and $\downarrow= \pm \infty, \pm \omega$, there is an isomorphism

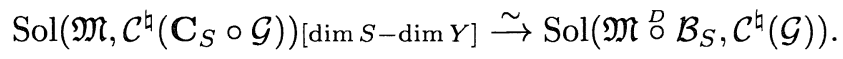

In order to apply this result, we are left to deal with two separate problems

(i) to calculate the $\mathcal{D}$-module transform $\mathfrak{M} \stackrel{D}{\circ} \mathcal{B}_{S}$,

(ii) to calculate the sheaf-theoretical transform $\mathbb{C}_{S} \circ \mathcal{G}$.

Problem (i) is of an analytic nature, and we will recall in the next section how it is solved in the case of the Radon transform. Concerning problem (ii), note 
that $g$ identifies the fiber of $f$ at $x \in X$ with the subset $\widehat{x} \subset Y$. Then, if $G \subset Y$ is a locally closed subset, one has

$$
\left(\mathbf{C}_{S} \circ \mathbf{C}_{G}\right)_{x} \simeq \mathrm{R} \Gamma_{c}(\widehat{x} \cap G ; \mathbf{C}) .
$$

In other words, the complex $\mathbf{C}_{S} \circ \mathbf{C}_{G}$ describes the topology of the slices $\widehat{x} \cap G$, when $x$ varies in $X$.

Before turning to the Radon transform, let us recall some miscellaneous results which will be of use in Section 3 .

- Let $Z$ be another manifold. With $\mathcal{K} \in \mathbf{D}^{\mathrm{b}}\left(\mathbf{C}_{X \times Y}\right)$ and $\mathcal{L} \in \mathbf{D}^{\mathrm{b}}\left(\mathbf{C}_{Y \times Z}\right)$ one associates

$$
\mathcal{K} \circ \mathcal{L}=R q_{13 !}\left(q_{12}^{-1} \mathcal{K} \otimes q_{23}^{-1} \mathcal{L}\right)
$$

Note that if $Z$ is reduced to a point, one recovers (2.2.2). One easily checks the associativity of the functor o, i.e.,

$$
(\mathcal{K} \circ \mathcal{L}) \circ \mathcal{H}=\mathcal{K} \circ(\mathcal{L} \circ \mathcal{H})
$$

for $\mathcal{H} \in \mathbf{D}^{\mathrm{b}}\left(\mathbf{C}_{Z \times W}\right)$. The same remarks apply for $\mathcal{D}$-modules.

- The functor $\circ$ is compatible with exterior products. More precisely, let $\tilde{X}, \tilde{Y}$ be another pair of complex analytic manifolds, and take $\mathcal{K} \in \mathbf{D}^{\mathrm{b}}\left(\mathbf{C}_{X \times Y}\right)$, $\widetilde{\mathcal{K}} \in \mathbf{D}^{\mathrm{b}}\left(\mathbf{C}_{\widetilde{X} \times \tilde{Y}}\right), \mathcal{G} \in \mathbf{D}^{\mathrm{b}}\left(\mathbf{C}_{Y}\right)$ and $\widetilde{\mathcal{G}} \in \mathbf{D}^{\mathrm{b}}\left(\mathbf{C}_{\tilde{Y}}\right)$. Then

$$
r_{*}(\mathcal{K} \otimes \widetilde{\mathcal{K}}) \circ(\mathcal{G} \otimes \widetilde{\mathcal{G}}) \simeq(\widetilde{\mathcal{K}} \circ \widetilde{\mathcal{G}}) \otimes(\mathcal{K} \circ \mathcal{G})
$$

where $r: X \times Y \times \tilde{X} \times \tilde{Y} \stackrel{\sim}{\rightarrow} X \times \tilde{X} \times Y \times \tilde{Y}$. The same remark applies to $\mathcal{D}$-modules.

- Consider for simplicity the case $\mathcal{K}=\mathbf{C}_{S}$, for $S \subset X \times Y$ a closed submanifold, and assume that the maps $f$ and $g$ in (2.2.4) are smooth and proper. Let $\mathcal{F} \in \mathbf{D}^{\mathrm{b}}\left(\mathbf{C}_{X}\right)$ and $\mathcal{G} \in \mathbf{D}^{\mathrm{b}}\left(\mathbf{C}_{Y}\right)$. Then, one has the adjunction formula (see [7, Proposition B.2])

$$
\operatorname{RHom}\left(\mathcal{F} \circ \mathbf{C}_{S}, \mathcal{G}\right) \simeq \operatorname{RHom}\left(\mathcal{F}, \mathbf{C}_{S} \circ \mathcal{G}\right)\left[\operatorname{dim}^{\mathbf{R}} S-\operatorname{dim}^{\mathbf{R}} Y\right],
$$

and the commutation with duality

$$
\left(\mathbf{C}_{S} \circ \mathcal{G}\right)^{\prime} \simeq \mathbb{C}_{S} \circ \mathcal{G}^{\prime}\left[\operatorname{dim}^{\mathbf{R}} S-\operatorname{dim}^{\mathbf{R}} X\right]
$$

- Following [20], we set

$$
\mathbf{D}_{\mathbf{R}-\mathrm{c}}^{\mathrm{b}}\left(X ; \dot{T}^{*} X\right)=\mathbf{D}_{\mathbf{R}-\mathrm{c}}^{\mathrm{b}}(X) / \mathcal{N},
$$

where $\mathcal{N}$ is the null system of objects $N \in \mathbf{D}_{\mathbf{R}-\mathrm{c}}^{\mathrm{b}}(X)$ whose micro-support $S S(N)$ is contained in the zero-section of $T^{*} X$. In other words, $\mathcal{F}=0$ in 
$\mathbb{D}_{\mathbf{R}-c}^{\mathrm{b}}\left(X ; \dot{T}^{*} X\right)$ if and only if its cohomology groups are locally constant. If $\mathcal{F}, \mathcal{G} \in \mathbb{D}_{\mathbf{R}-\mathrm{c}}^{\mathrm{b}}(X)$, we write

$$
\mathcal{F} \doteq \mathcal{G} \quad \text { if } \quad \mathcal{F} \simeq \mathcal{G} \text { in } \mathbb{D}_{\mathbf{R}-\mathrm{c}}^{\mathrm{b}}\left(X ; \dot{T}^{*} X\right) .
$$

\section{§2.3. Massless field equations}

Let us recall the construction of a distinguished family of $\mathcal{D}_{\mathbb{G}}$-modules attached to the correspondence (1.1.1).

The tautological bundle $U$ of $\mathbb{G}$ is the $(p+1)$-dimensional vector sub-bundle of the trivial bundle $\mathbb{G} \times \mathbb{V}$, whose fiber over a point of $\mathbb{G}$ is that same point considered as a subspace of $\mathbb{V}$. The quotient bundle $Q$ is defined by the exact sequence

$$
0 \rightarrow U \rightarrow \mathbb{G} \times \mathbb{V} \rightarrow Q \rightarrow 0 .
$$

Denoting by $\mathbb{V}^{*}$ the dual of $\mathbb{V}$, there is a natural identification $\mathbb{G} \simeq \operatorname{Gr}\left(n-p ; \mathbb{V}^{*}\right)$ obtained by associating with a $(p+1)$-dimensional subspace of $\mathbb{V}$ its orthogonal in $\mathbb{V}^{*}$. If $\zeta=\left(\zeta_{1}, \ldots, \zeta_{n-p}\right) \in \mathbb{V}^{* n-p}$ is an $(n-p)$-uple of independent vectors in $\mathbb{V}^{*}$, let us still denote by $\zeta$ the point of $\mathbb{G}$ corresponding to $\langle\zeta\rangle^{\perp} \subset \mathbb{V}$. One has

$$
U_{\zeta}=\langle\zeta\rangle^{\perp}, \quad U_{\zeta}^{*}=\mathbb{V}^{*} /\langle\zeta\rangle, \quad Q_{\zeta}=\mathbb{V} /\langle\zeta\rangle^{\perp}, \quad Q_{\zeta}^{*}=\langle\zeta\rangle .
$$

For example, as in [12], a section of $U^{\odot m} \otimes \operatorname{det} U$, where $\odot \operatorname{denotes}$ the symmetric tensor product, may be represented by a function $\psi\left(\zeta ; \sigma_{1}, \ldots, \sigma_{m}\right)$ such that

$$
\left\{\begin{array}{l}
\psi\left(\zeta ; \sigma_{1}, \ldots, \sigma_{m}\right) \text { is multilinear and symmetrical in } \sigma_{i} \in \mathbb{V}^{*} /\langle\zeta\rangle, \\
\psi\left(\mu \zeta ; \sigma_{1}, \ldots, \sigma_{m}\right)=(\operatorname{det} \mu)^{-1} \psi\left(\zeta ; \sigma_{1}, \ldots, \sigma_{m}\right) \text { for } \mu \in G L(n-p, \mathbb{C}) .
\end{array}\right.
$$

As another example, in the identification $T^{*} \mathbb{G} \simeq Q^{*} \otimes U$, the exterior differential reads

$$
\mathcal{O}_{\mathbb{G}} \underset{d}{\rightarrow} \Omega_{\mathbb{G}}^{1} \simeq \mathcal{O}_{\left(Q^{*} \otimes U\right),} \quad \psi(\zeta) \mapsto \chi(\zeta ; \sigma, t)=\left\langle\sigma \otimes t, \partial_{\zeta}\right\rangle \psi(\zeta),
$$

where $\chi$-which is a priori a section of the trivial bundle $\mathbb{G} \times\left(\mathbb{V} \otimes \mathbb{V}^{*}\right)$-is in fact a linear function of $\sigma \in \mathbb{V}^{*} /\langle\zeta\rangle$ and $t \in \mathbb{V} /\langle\zeta\rangle^{\perp}$, since $\psi(\mu \zeta)=\psi(\zeta)$ for $\mu \in G L(n-p, \mathbb{C})$.

For $m \in \mathbb{Z}$, the higher dimensional massless field equations of helicity $-(m+1) / 2$ are the differential operators

$$
\mathcal{O}\left(H_{m}\right) \underset{\square_{m}}{\longrightarrow} \mathcal{O}\left(\widetilde{H}_{m}\right)
$$

acting between sections of the vector bundles

$$
H_{m}= \begin{cases}U^{\odot-(m+1)} \otimes \operatorname{det} U & \text { for } m<-1 \\ \operatorname{det} U & \text { for } m=-1 \\ \left(Q^{*}\right)^{\odot(m+1)} \otimes \operatorname{det} U & \text { for } m>-1\end{cases}
$$


and (using Young tableaux)

$$
\tilde{H}_{m}= \begin{cases}Q^{*} \otimes U \overbrace{}^{* \wedge 2} \otimes \operatorname{det} U & \text { for } m<-1, \\ Q^{* \wedge 2} \otimes U^{\wedge 2} \otimes \operatorname{det} U & \text { for } m=-1, \\ Q^{*} \overbrace{}^{m+1} \otimes U \otimes \operatorname{det} U & \text { for } m>-1 .\end{cases}
$$

The section $\chi=\square_{m} \psi \in \mathcal{O}\left(\widetilde{H}_{m}\right)$ is defined by

$$
\begin{cases}\chi\left(\zeta ; \sigma_{0}, \ldots, \sigma_{-m-1}, t_{0}\right)=\left(\left\langle\sigma_{0} \otimes t_{0}, \partial_{\zeta}\right\rangle \psi\left(\zeta ; \sigma_{1}, \ldots, \sigma_{-m-1}\right)\right)_{\sigma_{0} \leftrightarrow \sigma_{1}} & \text { for } m<-1, \\ \chi\left(\zeta ; \sigma_{-1}, \sigma_{0}, t_{-1}, t_{0}\right)=\left(\left\langle\sigma_{-1} \otimes t_{-1}, \partial_{\zeta}\right\rangle\left\langle\sigma_{0} \otimes t_{0}, \partial_{\zeta}\right\rangle \psi(\zeta)\right)_{\sigma_{-1} \leftrightarrow \sigma_{0}} & \text { for } m=-1, \\ \chi\left(\zeta ; \sigma_{0}, t_{0}, \ldots, t_{m+1}\right)=\left(\left\langle\sigma_{0} \otimes t_{0}, \partial_{\zeta}\right\rangle \psi\left(\zeta ; t_{1}, \ldots, t_{m+1}\right)\right)_{t_{0} \leftrightarrow t_{1}} & \text { for } m>-1,\end{cases}
$$

where $\sigma_{k} \in \mathbb{V}^{*} /\langle\zeta\rangle, t_{\ell} \in \mathbb{V} /\langle\zeta\rangle^{\perp}$, and $\sigma_{0} \leftrightarrow \sigma_{1}$ denotes anti-symmetrization in $\sigma_{0}, \sigma_{1}$.

Fixing a basis of $\mathbb{V}^{*} \simeq \mathbf{C}^{n+1}$, the elements of the matrix $\left(\zeta_{k}^{\ell}\right)_{k=1, \ldots, n-p}^{\ell=0, \ldots, n}$ formed with the row vectors $\zeta_{k}=\left(\zeta_{k}^{0}, \ldots, \zeta_{k}^{n}\right)$, are called dual Stiefel coordinates of $\zeta \in \mathbb{G}$. For example, if $\psi(\zeta) \in \mathcal{O}_{\left(H_{-1}\right)}$, Maxwell's wave equation $\square_{-1} \psi=0$ reads

$$
\left(\partial_{\zeta_{k}^{\ell}} \partial_{\zeta_{k^{\prime}}^{\ell^{\prime}}}-\partial_{\zeta_{k}^{\ell^{\prime}}} \partial_{\zeta_{k^{\prime}}^{\ell}}\right) \psi(\zeta)=0, \quad \begin{aligned}
& \ell, \ell^{\prime}=0, \ldots, n \\
& k, k^{\prime}=1, \ldots, n-p .
\end{aligned}
$$

For $m \neq-1, \square_{m}$ is represented by a system of first order partial differential equations.

Remark. In Penrose's abstract index notations, one sets $\mathcal{O}_{A^{\prime}}=\mathcal{O}_{(U)}$,

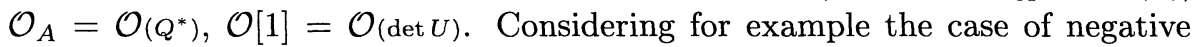
helicity $m>-1$, the above operators are written as (cf [2, Example 9.7.1])

$$
\begin{aligned}
\square_{m}: \mathcal{O}_{(\underbrace{B C \cdots D}_{m+1})}[1] & \rightarrow \mathcal{O}_{A^{\prime}[A(B] C \cdots D)}[1] \\
\phi_{B C \cdots D} & \mapsto \nabla_{A^{\prime}[A} \phi_{B] C \cdots D} .
\end{aligned}
$$

Denoting by Homdiff $\left(\mathcal{O}_{\left(H_{m}\right)}, \mathcal{O}_{\left(\widetilde{H}_{m}\right)}\right)$ the set of differential operators acting between section of the holomorphic vector bundles $\mathcal{O}_{\left(H_{m}\right)}$ and $\mathcal{O}\left(\widetilde{H}_{m}\right)$, one has an identification

$$
\operatorname{Homdiff}\left(\mathcal{O}_{\left(H_{m}\right)}, \mathcal{O}_{\left(\widetilde{H}_{m}\right)}\right) \simeq \operatorname{Hom}_{\mathcal{D}_{\mathbb{G}}}\left(\mathcal{D}\left(\widetilde{H}_{m}^{*}\right), \mathcal{D}\left(H_{m}^{*}\right)\right)
$$


where we set $\mathcal{D}\left(H_{m}^{*}\right)=\mathcal{D}_{\mathbb{G}} \otimes_{\mathcal{O}_{\mathbb{G}}} \mathcal{O}_{\left(H_{m}^{*}\right)}$. We still denote by $\square_{m}$ the associated $\mathcal{D}_{\mathbb{G}}$-module defined by

$$
\operatorname{coker}\left(\mathcal{D}\left(\widetilde{H}_{m}^{*}\right) \underset{\square_{m}}{\longrightarrow} \mathcal{D}\left(H_{m}^{*}\right)\right)
$$

In particular,

$$
\square_{m^{*}}=\mathcal{D}_{\mathbb{P}}\left(-m^{*}\right)=\mathcal{D}_{\mathbb{P}} \otimes_{\mathcal{O}_{\mathbb{P}}} \mathcal{O}_{\mathbb{P}}\left(-m^{*}\right) \quad \text { for } p=0, m^{*}<0 .
$$

As stated in the next theorem, the Radon-Penrose transform with kernel $\mathcal{B}_{\mathbb{F}}$ realizes $\square_{m}$ as the image of the locally free $\mathcal{D}_{\mathbb{P}}$-module of rank one $\mathcal{D}_{\mathbb{P}}\left(-m^{*}\right)$.

Theorem 2.3.1. For $m^{*}<0$ the Radon correspondence (1.1.1) can be quantized to give a $\mathcal{D}_{\mathbb{G}}$-linear isomorphism

$$
\square_{m} \stackrel{\sim}{\rightarrow} \mathcal{D}_{\mathbb{P}}\left(-m^{*}\right) \stackrel{D}{\circ} \mathcal{B}_{\mathbb{F}}
$$

For $n=3$ and $p=1$ the above isomorphism was obtained in [6], using the results in [10]. Similarly, the general case can be obtained using the results in [2]. The more satisfactory approach via quantization, which is needed for the description of integral kernels, is discussed in [7] for $p=n-1$, and in [5] for $p<n-1$. In the framework of twisted equivariant $\mathcal{D}$-modules, a proof of the above isomorphism for $m=-1$ is given in [27] (see also [24]).

\section{§2.4. Radon adjunction formula}

Combining Corollary 2.2.2 and Theorem 2.3.1, we get

Theorem 2.4.1. For $m^{*}<0, \mathcal{G} \in \mathbf{D}_{\mathbf{R}-\mathrm{c}}^{\mathrm{b}}\left(\mathbf{C}_{\mathbb{G}}\right)$, and $\natural= \pm \infty$, $\pm \omega$, the Radon correspondence (1.1.1) induces an isomorphism

$$
\mathrm{R} \Gamma\left(\mathbb{P} ; \mathcal{C}^{\natural}\left(\mathbf{C}_{\mathbb{F}} \circ \mathcal{G}\right)\left(m^{*}\right)\right)[p] \simeq \operatorname{Sol}\left(\square_{m}, \mathcal{C}^{\natural}(\mathcal{G})\right) .
$$

The above isomorphism holds in the derived category, while most of the results that we stated in the first section deals only with the first non-vanishing cohomology group. Thus, we now give some technical results that allows one to get an isomorphism

$$
H^{0}\left(\mathbb{P} ; \mathcal{C}^{\natural}(\mathcal{F})\left(m^{*}\right)\right) \simeq H^{0} \operatorname{Sol}\left(\square_{m}, \mathcal{C}^{\natural}(\mathcal{G})\right)
$$

with hypotheses weaker than $\mathcal{F} \simeq \mathbf{C}_{\mathbb{F}} \circ \mathcal{G}[p]$.

Let $\mathcal{C}$ and $\mathcal{C}^{\prime}$ be two abelian categories, and $\Phi: \mathbb{D}^{+}(\mathcal{C}) \rightarrow \mathbb{D}^{+}\left(\mathcal{C}^{\prime}\right)$ a triangulated functor. Recall that $\Phi$ is said to be left exact (for the natural $t$-structure) if $\Phi\left(\mathbb{D}^{\geq 0}(\mathcal{C})\right) \subset \mathbb{D}^{\geq 0}\left(\mathcal{C}^{\prime}\right)$. For example, the right derived functor $R F$ of a left exact functor $F: \mathcal{C} \rightarrow \mathcal{C}^{\prime}$ is left exact. One denotes by $\tau^{\leq r}$ and $\tau^{\geq r}$ the truncation functors in derived categories.

Lemma 2.4.2. Let $\mathcal{C}$ and $\mathcal{C}^{\prime}$ be two abelian categories, $\Phi: \mathbb{D}^{+}(\mathcal{C}) \rightarrow$ $\mathrm{D}^{+}\left(\mathcal{C}^{\prime}\right)$ a left exact functor, and $\mathcal{N}$ a null system in $\mathbb{D}^{+}(\mathcal{C})$. Assume that 
(a) for some $s \geq 2$ and for any $N \in \mathcal{N} \cap \mathbf{D}^{\geq 0}(\mathcal{C})$, one has $\Phi(N) \stackrel{\sim}{\rightarrow} \tau^{\geq s} \Phi(N)$.

Let $X, Y \in \mathbf{D}^{\geq 0}(\mathcal{C})$, and assume that

(b) for some $r \leq s-1$ there is a distinguished triangle $Y \rightarrow \tau^{\leq r} X \rightarrow N \underset{+1}{\longrightarrow}$, with $N \in \mathcal{N} \cap \mathbf{D}^{\geq 0}(\mathcal{C})$.

Then

$$
\tau^{\leq r} \Phi(X) \stackrel{\sim}{\longleftarrow} \tau^{\leq r} \Phi(Y)
$$

Proof. Consider the distinguished triangle

$$
Y \rightarrow \tau^{\leq r} X \rightarrow N \underset{+1}{\longrightarrow}
$$

of hypothesis (b). By hypothesis (a), $\tau^{<s} \Phi(N)=0$. Consider the distinguished triangle $\Phi(Y) \rightarrow \Phi\left(\tau^{\leq r} X\right) \rightarrow \Phi(N) \underset{+1}{\longrightarrow}$. Since $r \leq s-1$, one deduces that $\tau^{\leq r} \Phi(Y) \stackrel{\sim}{\longrightarrow} \tau^{\leq r} \Phi\left(\tau^{\leq r} X\right)$. The statement follows since $\Phi$ is left exact.

Recall the notation $\mathcal{F} \doteq \mathcal{G}$ of (2.2.11).

Corollary 2.4.3. Let $\mathcal{F}$ be an $\mathbf{R}$-constructible sheaf on $\mathbb{P}$, and $\mathcal{G}$ an $\mathbf{R}$ constructible sheaf on $\mathbb{G}$. Assume that $\tau \leq p\left(\mathbf{C}_{\mathbb{F}} \circ \mathcal{G}\right) \doteq \mathcal{F}[-p]$. Then, for $m^{*}<0$ and $\emptyset= \pm \infty, \pm \omega$ the Radon correspondence (1.1.1) induces an isomorphism

$$
H^{0}\left(\mathbb{P} ; \mathcal{C}^{\natural}(\mathcal{F})\left(m^{*}\right)\right) \simeq H^{0} \operatorname{Sol}\left(\square_{m}, \mathcal{C}^{\natural}(\mathcal{G})\right)
$$

Proof. By (2.4.1), we have to prove

$$
\tau^{\leq p} \mathrm{R} \Gamma\left(\mathbb{P} ; \mathcal{C}^{\natural}(\mathcal{F}[-p])\left(m^{*}\right)\right) \simeq \tau^{\leq p} \mathrm{R} \Gamma\left(\mathbb{P} ; \mathcal{C}^{\natural}\left(\mathbf{C}_{\mathbb{F}} \circ \mathcal{G}\right)\left(m^{*}\right)\right) .
$$

This follows from Lemma 2.4.2, for $X=\mathbf{C}_{\mathbb{F}} \circ \mathcal{G}, Y=\mathcal{F}_{[-p]}, \Phi=\mathrm{R} \Gamma\left(\mathbb{P} ; \mathcal{C}^{\natural}(\cdot)\left(m^{*}\right)\right)$, $\mathcal{N}=\left\{\mathcal{F} \in \mathbf{D}_{\mathbf{R}-\mathrm{c}}^{\mathrm{b}}\left(\mathbf{C}_{\mathbb{P}}\right): \mathcal{F} \doteq 0\right\}, s=n$, and $r=p$. (Note that hypothesis (a) in Lemma 2.4.2 follows from Serre's isomorphism (3.3.8).)

The following result will be of use.

Lemma 2.4.4. Let $\mathcal{C}$ and $\mathcal{C}^{\prime}$ be two abelian categories, and $\Phi: \mathbf{D}^{+}(\mathcal{C}) \rightarrow$ $\mathbf{D}^{+}\left(\mathcal{C}^{\prime}\right)$ a left exact functor. Let $X \in \mathbf{D}^{\geq 0}(\mathcal{C}), Y \in \mathcal{C}$, and assume that for some $r \geq 0$ one has $\tau^{<r} \Phi(X)=0$, and $H^{j}(X) \simeq Y^{\ell_{3}}$ for $j \leq r$, with $\ell_{0} \neq 0$. Then

$$
\tau^{<r} \Phi(Y)=0, \quad H^{r}(\Phi(X)) \simeq H^{r}(\Phi(Y))^{\ell_{0}} .
$$


Proof. The statement is obvious if $r=0$, so we may assume $r>0$. As short hand notations, set $X_{j}=\tau^{\geq j} X, \Phi^{j}(\cdot)=H^{j}(\Phi(\cdot))$. Consider the distinguished triangles

$$
\Phi(Y)^{\ell_{j}}[-j] \rightarrow \Phi\left(X_{j}\right) \rightarrow \Phi\left(X_{j+1}\right) \underset{+1}{\longrightarrow} .
$$

For $j=0$, part of the associated long exact cohomology sequence reads

$$
\Phi^{r-1} X_{1} \rightarrow \Phi^{r} Y^{\ell_{0}} \rightarrow \Phi^{r} X \rightarrow \Phi^{r} X_{1},
$$

and hence it is sufficient to prove that $\Phi^{r-1} X_{1}=\Phi^{r} X_{1}=0$. In fact, we will show that for $0 \leq i \leq r-1$ the following statement holds

$$
\mathcal{P}(i):\left\{\begin{array}{l}
\Phi^{i} Y=0, \\
\Phi^{k+i} X_{k}=0 \quad \text { for any } 1 \leq k \leq r-i .
\end{array}\right.
$$

For $i=0$, we have to prove that $\Phi^{0} Y=\Phi^{k} X_{k}=0$ for any $1 \leq k \leq r$. This is true, since the beginning of the long exact cohomology sequence associated with (2.4.3) for $j=0$ reads

$$
0 \rightarrow \Phi^{0} Y^{\ell_{0}} \rightarrow \Phi^{0} X=0
$$

and moreover $\Phi^{k} X_{k}=\Phi^{0} Y^{\ell_{k}}$ since $\Phi$ is left exact.

Assuming that $\mathcal{P}(j)$ holds for $j<i$, let us show that $\mathcal{P}(i)$ holds. Similarly as above, there is an exact sequence

$$
\Phi^{i-1} X_{1} \rightarrow \Phi^{i} Y^{\ell_{0}} \rightarrow \Phi^{i} X=0 .
$$

Since $\Phi^{i-1} X_{1}=0$ by $\mathcal{P}(i-2)$, this implies $\Phi^{i} Y=0$. Finally, for $k$ such that $1 \leq k \leq r-i$, consider the exact sequence

$$
\Phi^{i} Y^{\ell_{k}} \rightarrow \Phi^{k+i} X_{k} \rightarrow \Phi^{k+i} X_{k+1} .
$$

We just proved that $\Phi^{\imath} Y=0$, and moreover $\Phi^{k+i} X_{k+1}=0$ by $\mathcal{P}(i-1)$.

\section{§3. Proofs and Further Results}

\section{§3.1. The Radon-Penrose transform}

Proof of Proposition 1.1.1. By definition, one has

$$
\mathcal{C}^{-\omega}\left(\mathbb{C}_{\widehat{U}}^{\prime}\right) \simeq \Gamma_{\widehat{U}} \mathcal{O}_{\mathbb{P}}, \quad \mathcal{C}^{-\omega}\left(\mathbf{C}_{U}^{\prime}\right) \simeq \Gamma_{U} \mathcal{O}_{\mathbb{G}}
$$

In particular, the isomorphism in the statement of Proposition 1.1.1 can be rewritten as

$$
H^{0}\left(\mathbb{P} ; \mathcal{C}^{-\omega}\left(\mathbf{C}_{\widehat{U}}^{\prime}[p]\right)\left(m^{*}\right)\right) \simeq H^{0} \operatorname{Sol}\left(\square_{m}, \mathcal{C}^{-\omega}\left(\mathbf{C}_{U}^{\prime}\right)\right)
$$


By Corollary 2.4.3, it is then enough to show that

$$
\tau^{\leq p}\left(\mathbf{C}_{\mathbb{F}} \circ \mathbf{C}_{U}^{\prime}\right) \simeq \mathbf{C}_{\widehat{U}}^{\prime}
$$

By (2.2.9), this is in turn equivalent to the isomorphism

$$
\tau^{\geq 2 p(n-p)-p}\left(\mathbf{C}_{\mathbb{F}} \circ \mathbf{C}_{U}\right) \simeq \mathbf{C}_{\widehat{U}}^{[-2 p(n-p)]},
$$

which is proved in the next lemma.

The following result is a slight extension of [6, Lemma 2.8]

Lemma 3.1.1. If $U \subset \mathbb{G}$ is $\mathbb{F}$-elementary, then

$$
\tau^{\geq 2 p(n-p)-p}\left(\mathbf{C}_{\mathbb{F}} \circ \mathbf{C}_{U}\right) \simeq \mathbf{C}_{\widehat{U}}^{[-2 p(n-p)]} .
$$

Proof. For $z \in \widehat{U}$, consider the natural morphism

$$
\rho: \mathbf{C} \rightarrow \mathrm{R} \Gamma(\widehat{z} \cap U ; \mathbf{C}) .
$$

By definition, $U \subset \mathbb{G}$ is $\mathbb{F}$-elementary if and only if $H^{\jmath}(\rho)$ is an isomorphism for $j \leq p$. Note that $\widehat{z} \cap U$ is an open subset of the complex manifold $\widehat{z} \simeq$ $\operatorname{Gr}(p ; \mathbb{W} / z)$, which has dimension $p(n-p)$. By Poincaré-Verdier duality, this is equivalent to say that the dual morphism

$$
\rho^{*}: \mathrm{R} \Gamma_{c}(\widehat{z} \cap U ; \mathbf{C})[2 p(n-p)] \rightarrow \mathbf{C}
$$

induces isomorphisms $H^{\jmath}\left(\rho^{*}\right)$ for $j \geq-p$. One concludes by noticing that $\rho^{*}$ is the fiber at $z$ of the adjunction morphism

$$
\mathbf{C}_{\mathbb{F}} \circ \mathbf{C}_{U}^{[2 p(n-p)]} \simeq R f_{!} \mathbf{C}_{g^{-1}(U)}[2 p(n-p)] \simeq R f_{!} f^{!} \mathbf{C}_{\widehat{U}} \rightarrow \mathbf{C}_{\widehat{U}}
$$

\section{§3.2. Action of $S U(p+1, n-p)$}

\subsubsection{Conformal case.}

As in Section 1.2.1, let $\omega$ be a nondegenerate hermitian form on $\mathbb{V}$ of signature $(p+1, n-p)$, and denote by $\mathrm{P} \subset \mathbb{P}$ and $\mathrm{G} \subset \mathbb{G}$ the submanifolds of isotropic lines and planes, respectively.

Proof of Proposition 1.2.1. We have to show that the horizontal rows of the commutative diagram

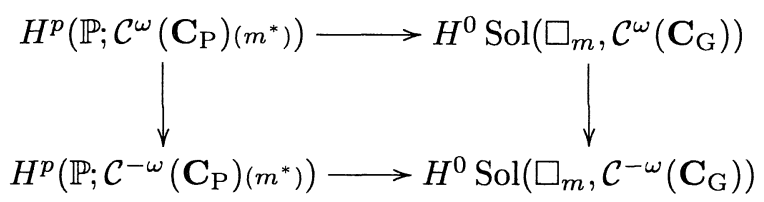


are isomorphisms. Concerning the top row, by Theorem 2.4.1 we are left to show that

$$
H^{p}\left(\mathbb{P} ; \mathcal{C}^{\omega}\left(\mathbf{C}_{\mathrm{P}}\right)\left(m^{*}\right)\right) \simeq H^{p}\left(\mathbb{P} ; \mathcal{C}^{\omega}\left(\mathbf{C}_{\mathbb{F}} \circ \mathbf{C}_{\mathrm{G}}\right)\left(m^{*}\right)\right) .
$$

$\operatorname{Set} \mathcal{C}=\operatorname{Mod}\left(\mathbf{C}_{\mathbb{P}}\right), \mathcal{C}^{\prime}=\operatorname{Mod}(\mathbf{C}), \Phi=\mathrm{R} \Gamma\left(\mathbb{P} ; \mathcal{C}^{\omega}(\cdot)\left(m^{*}\right)\right), X=\mathbf{C}_{\mathbb{F}} \circ \mathbf{C}_{\mathrm{G}}, Y=\mathbf{C}_{\mathrm{P}}$, $r=p$, and $\ell_{0}=1$. Theorem 2.4.1 implies that $\tau^{<r} \Phi(X)=0$, while Proposition 3.2.1 below ensures that $H^{j}(X) \simeq Y^{\ell_{3}}$. Then, the above isomorphism follows from Lemma 2.4.4.

Similarly, in order to prove that the bottom line of the above diagram is an isomorphism, it is enough to show that

$$
H^{p}\left(\mathbb{P} ; \mathcal{C}^{-\omega}\left(\mathbf{C}_{\mathrm{P}}\right)\left(m^{*}\right)\right) \simeq H^{p}\left(\mathbb{P} ; \mathcal{C}^{-\omega}\left(\mathbf{C}_{\mathbb{F}} \circ \mathbf{C}_{\mathrm{G}}\right)\left(m^{*}\right)\right)
$$

i.e., that

$$
H^{p+1} \mathrm{RHom}\left(\mathbf{C}_{\mathrm{P}}^{\prime}{ }^{[1]}, \mathcal{O}_{\mathbb{P}}\left(m^{*}\right)\right) \simeq H^{p+1} \mathrm{RHom}\left(\left(\mathbf{C}_{\mathbb{F}} \circ \mathbf{C}_{\mathrm{G}}\right)^{\prime}[1], \mathcal{O}_{\mathbb{P}\left(m^{*}\right)}\right) .
$$

This is a consequence of Lemma 2.4.4, for $\mathcal{C}=\operatorname{Mod}\left(\mathbf{C}_{\mathbb{P}}\right)^{\text {opp }}$, the opposite category, $\mathcal{C}^{\prime}=\operatorname{Mod}(\mathbf{C}), \Phi=\operatorname{RHom}\left(\cdot, \mathcal{O}_{\mathbb{P}}\left(m^{*}\right)\right), X=\left(\mathbf{C}_{\mathbb{F}} \circ \mathbf{C}_{\mathrm{G}}\right)^{\prime}[1], Y=\mathbf{C}_{\mathrm{P}}^{\prime}{ }^{[1]}$, $r=p+1$, and $\ell_{0}=1$. To check that the hypotheses of Lemma 2.4 .4 are satisfied, note the following facts. Since $\mathrm{P} \subset \mathbb{P}$ is a hypersurface, $\mathbb{C}_{\mathrm{P}}^{\prime}[1] \simeq \mathbf{C}_{\mathrm{P}}$. By Lemma 3.2.2 (ii), $\mathbf{C}_{\mathrm{G}}^{\prime} \simeq \mathbf{C}_{\mathrm{G}}\left[-(p+1)^{2}\right]$. Moreover, by (2.2.9),

$$
\begin{aligned}
\left(\mathbf{C}_{\mathbb{F}} \circ \mathbf{C}_{\mathrm{G}}\right)^{\prime} & \simeq \mathbf{C}_{\mathbb{F}} \circ \mathbf{C}_{\mathrm{G}}^{\prime}[2 p(n-p)] \\
& \simeq \mathbf{C}_{\mathbb{F}} \circ \mathbf{C}_{\mathrm{G}}[p(2 n-3 p-2)-1],
\end{aligned}
$$

so that $\left(\mathbf{C}_{\mathbb{F}} \circ \mathbf{C}_{G}\right)^{\prime}[1]$ is in degree $\leq 0$ by Proposition 3.2.1.

Remark. More generally, consider a nondegenerate hermitian form $\omega$ of signature $(q+1, n-q)$, with $p+1 \leq q+1 \leq(n+1) / 2$. Using the above arguments, one could prove that there is a commutative diagram

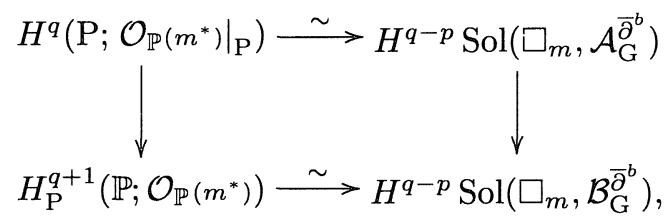

and that the lower cohomology groups vanish.

Proposition 3.2.1. With the above notations, one has

$$
H^{k}\left(\mathbf{C}_{\mathbb{F}} \circ \mathbf{C}_{\mathrm{G}}\right) \simeq \mathbf{C}_{\mathrm{P}}^{\ell_{k}},
$$

where $\ell_{0}=1$, and $\ell_{k}=0$ for $k>p(2 n-3 p-2)$. 
Proof. Consider the double fibration

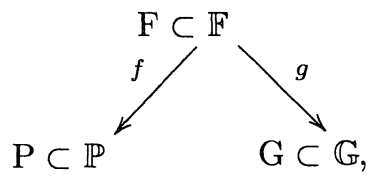

where $\mathrm{F}=g^{-1}(\mathrm{G})$, and note that $\mathrm{P}=\widehat{\mathrm{G}}$. Since $\mathbf{C}_{\mathbb{F}} \circ \mathbf{C}_{\mathrm{G}}=R f_{!} \mathbf{C}_{\mathrm{F}}$, the statement is a corollary of the following Lemma, noticing that $p(2 n-3 p-2)=$ $\sum_{k=0}^{p-1}(2(n-p-1-k)-1)$.

Lemma 3.2.2. Assume that $p+1 \leq n-p$. Then

(i) $\mathrm{P}$ is a simply connected, quadratic hypersurface of $\mathbb{P}$,

(ii) G is a generic, affine, orientable submanifold of codimension $(p+1)^{2}$ in $\mathbb{G}$,

(iii) The projection $\left.f\right|_{\mathrm{F}}: \mathrm{F} \rightarrow \mathrm{P}$ decomposes into sphere bundles $\mathrm{F} \underset{f_{p-1}}{\longrightarrow} \mathrm{F}_{p-1} \rightarrow$ $\cdots \mathrm{F}_{1} \underset{f_{0}}{\longrightarrow} \mathrm{P}$, where the fiber dimension of $f_{k}$ is $2(n-p-1-k)-1$.

Proof. (i) By definition, $z \in \mathrm{P}$ if and only if it is $\omega$-isotropic. Take a basis of $\mathbb{V}$, so that

$$
\omega=\left(\begin{array}{cc}
1_{p+1} & 0 \\
0 & -1_{n-p}
\end{array}\right)
$$

and let $[z]=\left[z^{\prime}, z^{\prime \prime}\right]$ be the corresponding homogeneous coordinates in $\mathbb{P}$. Then, $\mathrm{P}$ is defined by the quadratic equation $\left|z^{\prime}\right|=\left|z^{\prime \prime}\right|$. Moreover, the map $\left[z^{\prime}, z^{\prime \prime}\right] \mapsto$ $\left[z^{\prime}\right]$ induces a fibration of $\mathrm{P}$ over a $p$-dimensional complex projective space, whose fibers are $(2(n-p)-1)$-dimensional spheres. Since $2(n-p)-1 \geq$ $2(p+1)-1 \geq 3$, we get that $\mathrm{P}$ is simply connected.

(ii) As we already noticed, orbits of real forms of complex Lie groups are always generic. Let $[\zeta]=\left[\zeta^{\prime}, \zeta^{\prime \prime}\right]$ be the Stiefel coordinates in $\mathbb{G}$ associated with the above choice of basis, where $\zeta^{\prime}$ and $\zeta^{\prime \prime}$ are $(p+1) \times(p+1)$ and $(p+1) \times(n-p)$ matrices, respectively. Then, $\mathrm{G}$ is defined by the homogeneous system of equations $\zeta^{\prime} \zeta^{\prime *}=\zeta^{\prime \prime} \zeta^{\prime \prime *}$. In particular, $\zeta^{\prime}$ is invertible, since otherwise there would be $\lambda \in G L(p+1, \mathbf{C})$ such that $\widetilde{\zeta}=\lambda \zeta$ contains a row vector $\widetilde{\zeta}_{k}=\left(0, \widetilde{\zeta}_{k}^{\prime \prime}\right)$, which is not $\omega$-isotropic. Hence, $\mathrm{G}$ is contained in the affine chart of $\mathbb{G}$ defined by $\operatorname{det}\left(\zeta^{\prime}\right) \neq 0$, which is endowed with the system of coordinates $\left(\zeta^{\prime \prime}\right)$. In this chart, $\mathrm{G}$ is defined by $\zeta^{\prime \prime} \zeta^{\prime \prime *}=1_{p+1}$, which gives a system of $(p+1)^{2}$ independent real equations. In particular, $\mathrm{G}$ is orientable.

Denoting by $\zeta_{k}^{\prime \prime}$ the row vectors of the matrix $\left(\zeta^{\prime \prime}\right)$, these equations read $\left\langle\bar{\zeta}_{k}^{\prime \prime}, \zeta_{l}^{\prime \prime}\right\rangle=\delta_{k, l}$. Thus, the maps $\zeta^{\prime \prime}=\left(\zeta_{1}^{\prime \prime}, \ldots, \zeta_{p+1}^{\prime \prime}\right) \mapsto\left(\zeta_{1}^{\prime \prime}, \ldots, \zeta_{p}^{\prime \prime}\right) \mapsto \cdots \mapsto$ 
$\zeta_{1}^{\prime \prime}$ define sphere bundles $\mathrm{G}=\mathrm{G}_{p+1} \underset{q_{p}}{\longrightarrow} \mathrm{G}_{p} \rightarrow \cdots \underset{q_{1}}{\rightarrow} \mathrm{G}_{1}$, where the fiber dimension of $q_{k}$ is $2(n-p-k)-1$, and $\mathrm{G}_{1}$ is a sphere of dimension $2(n-p)-1$.

Recall that if $\eta$ is a $d$-dimensional $\omega$-isotropic subspace of $\mathbb{V}$, with $d \leq p+1$, then the space $\mathbb{V}^{\eta}=\eta^{\perp} / \eta$ is naturally endowed with the form $\omega^{\eta}(v+\eta, w+\eta)=$ $\omega(v, w)$ of signature $(p+1-d, n-p-d)$. The correspondence

$$
\zeta \mapsto \zeta^{\eta}=\left[\left(\zeta \cap \eta^{\perp}\right)+\eta\right] / \eta
$$

satisfies $\left(\zeta^{\eta}\right)^{\perp}=\left(\zeta^{\perp}\right)^{\eta}$, and hence sends $\omega$-isotropic subspaces of $\mathbb{V}$ to $\omega^{\eta}$ isotropic subspaces of $\mathbb{V}^{\eta}$. In particular, there is a bijection between points $(z, \zeta) \in \mathrm{F}$ and pairs $\left(z, \zeta^{z}\right)$, where $\zeta^{z}$ is an isotropic $p$-dimensional plane in $\mathbb{V}^{z} \simeq$ $\mathrm{C}^{n-1}$. This shows that $f$ is a fiber bundle over $\mathrm{P}$, whose fiber is the Grassmann manifold of isotropic $p$-dimensional planes in $\mathbb{V}^{z}$. Finally, a construction similar to the one in the previous paragraph proves that this bundle can be decomposed in a chain of sphere bundles with the claimed fiber dimensions.

\subsubsection{Conformal affine case.}

As in Section 1.2.2, let $\zeta_{\circ} \in \mathrm{G}$, denote by $\mathbb{G}_{\circ} \subset \mathbb{G}$ the open subset of planes transversal to $\zeta_{\circ}^{\perp}$, and set $G_{\circ}=G \cap \mathbb{G}_{\circ}$. Similarly, consider $\mathbb{P}_{\circ} \subset \mathbb{P}$ and $\mathrm{P}_{\mathrm{o}}=\mathrm{P} \cap \mathbb{P}_{\mathrm{o}}$.

Proof of Proposition 1.2.2. We have to prove that

$$
H^{p}\left(\mathbb{P} ; \mathcal{C}^{-\omega}\left(\mathbf{C}_{\mathrm{P}_{\circ}}\right)\left(m^{*}\right)\right) \stackrel{\sim}{\longrightarrow} H^{0} \operatorname{Sol}\left(\square_{m}, \mathcal{C}^{-\omega}\left(\mathbf{C}_{\mathrm{G}_{\circ}}\right)\right) .
$$

By Proposition 3.2.3 below, this is a consequence of Theorem 2.4.1.

As for the extendability of solutions, using the above result and Proposition 1.2.1 we get a commutative diagram

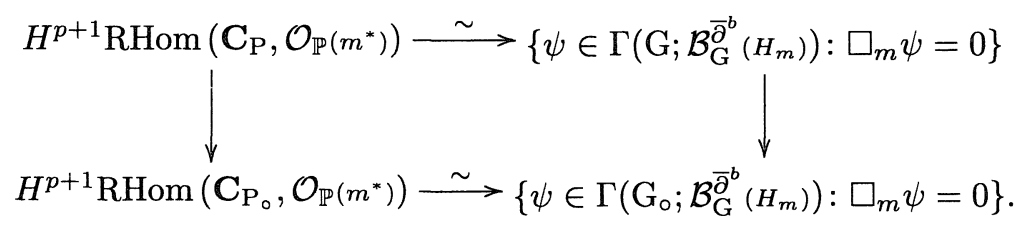

It is then enough to prove that

$$
H^{p+2} \mathrm{RHom}\left(\mathbf{C}_{\mathrm{P} \backslash \mathrm{P}_{\circ}}, \mathcal{O}_{\left.\mathbb{P}\left(m^{*}\right)\right)}=0 .\right.
$$

By definition, $\mathrm{P} \backslash \mathrm{P}_{\circ}$ is the set of $\omega$-isotropic lines in $\zeta_{\circ}^{\perp}$, and since any line is isotropic, it is identified with a $p$-dimensional complex projective space. Let us set $\mathbb{L}=\mathrm{P} \backslash \mathrm{P}$ 。 for short, and consider the distinguished triangle

$$
\operatorname{RHom}\left(\mathbf{C}_{\mathbb{L}}, \mathcal{O}_{\mathbb{P}}\left(m^{*}\right)\right) \rightarrow \operatorname{RHom}\left(\mathbf{C}_{\mathbb{P}}, \mathcal{O}_{\mathbb{P}}\left(m^{*}\right)\right) \rightarrow \operatorname{RHom}\left(\mathbf{C}_{\mathbb{P} \backslash \mathbb{L}}, \mathcal{O}_{\mathbb{P}}\left(m^{*}\right)\right) \underset{+1}{\longrightarrow}
$$


As we recalled in Example 1.1.3, $\mathbb{P} \backslash \mathbb{L}$ is $p$-linearly concave, and hence the last complex vanishes in degree smaller than $n-p-1$. Moreover, $\mathbb{P} \backslash \mathbb{L}$ is covered by the $n-p$ Stein open subsets $\mathbb{P} \backslash \mathbb{H}_{\jmath}$, where $\mathbb{H}_{\jmath}$ is the hyperplane $z_{p+\jmath}=0$ in the system of homogeneous coordinates where $\mathbb{L}$ is defined by $z_{p+1}=\cdots=z_{n+1}=0$. It follows that the last complex is concentrated in degree $n-p-1$. Since $m^{*}<0$, the second complex is concentrated in degree $n$. We can then conclude, unless $p+1=n-p-1$.

Proposition 3.2.3. With the above notations, one has

$$
\mathbf{C}_{\mathbb{F}} \circ \mathbf{C}_{\mathrm{G}_{\circ}} \simeq \mathbf{C}_{\mathrm{P}_{\circ}[-p(2 n-3 p-2)]} \text {. }
$$

Proof. Setting $\mathrm{F}_{\circ}=g^{-1}\left(\mathrm{G}_{\circ}\right)$, we have $\widehat{\mathrm{G}_{\circ}}=\mathrm{P}_{\circ}$, and $\mathbf{C}_{\mathrm{F}} \circ \mathbf{C}_{\mathrm{G}_{\circ}} \simeq R f_{!} \mathbf{C}_{\mathrm{F}_{\circ}}$. The statement is then a corollary of the following Lemma.

Lemma 3.2.4. Assume that $p+1 \leq n-p$. Then

(i) $\mathrm{P}_{\circ}$ is an open simply connected subset of $\mathrm{P}$,

(ii) $\mathrm{G}_{\circ} \simeq \mathbf{R}^{(p+1)(2 n-3 p-1)}$ is an affine chart in $\mathrm{G}$,

(iii) The map $\left.f\right|_{\mathrm{F}_{\circ}}: \mathrm{F}_{\circ} \rightarrow \mathrm{P}_{\circ}$ is an $\mathbf{R}^{p(2 n-3 p-2)}$-bundle.

Proof. Recall that $\mathrm{P} \backslash \mathrm{P}_{\circ}$ is a $p$-dimensional complex projective space. In particular, $\mathrm{P} \backslash \mathrm{P} \circ$ has codimension $2 n-1-2 p \geq 2 p+1 \geq 3$ in $\mathrm{P}$. Since $\mathrm{P}$ is simply connected, so is $\mathrm{P}_{\circ}$.

To prove (ii), choose a basis $\left\{e_{1}, \ldots, e_{n+1}\right\}$ of $\mathbb{V}$ so that $\zeta_{\circ}$ is the complex plane $\left\langle e_{p+2}, \ldots, e_{2(p+1)}\right\rangle_{\mathbf{C}}$ generated by $e_{p+2}, \ldots, e_{2(p+1)}$, and

$$
\omega=\left(\begin{array}{ccc}
0 & i 1_{p+1} & 0 \\
-i 1_{p+1} & 0 & 0 \\
0 & 0 & 1_{n-2 p-1}
\end{array}\right) .
$$

In particular, $\zeta_{\circ}^{\perp}=\left\langle e_{p+2}, \ldots, e_{n+1}\right\rangle_{\mathbf{C}}$. In the associated system of Stiefel coordinates $\left[\zeta^{\prime}, \zeta^{\prime \prime}, \zeta^{\prime \prime \prime}\right], \mathbb{G}_{\circ}$ is described by the inequality $\operatorname{det} \zeta^{\prime} \neq 0$, so that $\left(1_{p+1}, \zeta^{\prime}, \zeta^{\prime \prime}\right)$ is a system of coordinates in $\mathbb{G}_{0}$. (Note that $\zeta^{\prime \prime}$ and $\zeta^{\prime \prime \prime}$ are $(p+1) \times(p+1)$ and $(p+1) \times(n-2 p-1)$ matrices, respectively.) In this system of coordinates, the isotropy condition defining $G_{\circ} \subset \mathbb{G}_{\circ}$ reads $\zeta^{\prime}-\zeta^{*}=$ $-i \zeta^{\prime \prime} \zeta^{\prime \prime *}$. This is a system of $(p+1)^{2}$ linear independent conditions on the antihermitian part of $\zeta^{\prime}$. It follows that $\mathrm{G}_{\circ} \subset \mathrm{G}$ is a real affine chart of dimension $2\left((p+1)^{2}+(p+1)(n-2 p-1)\right)-(p+1)^{2}=(p+1)(2 n-3 p-1)$.

For $z \in \mathrm{P}_{\circ}, f^{-1}(z) \cap g^{-1}\left(\mathrm{G}_{\circ}\right)$ is the family of $\omega$-isotropic $(p+1)$-subspaces of $\mathbb{V}$ containing $z$ and transversal to $\zeta_{\circ}^{\perp}$. The map $\zeta \mapsto \zeta^{z}$ of $(3.2 .1)$, identifies it to the family of $\omega^{z}$-isotropic $p$-subspaces of $\mathbb{V}^{z}$ transversal to $\left(\zeta_{\circ}^{z}\right)^{\perp}$. The construction in the previous paragraph shows that this set is in turn identified with $\mathbf{R}^{p(2 n-3 p-2)}$. 


\section{§3.3. Action of $S L(n+1, \mathbf{R})$}

With the notations of section 1.3.1, let us consider the double fibration

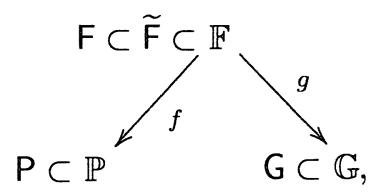

where $F=\mathbb{F} \cap(P \times G)$, and $\widetilde{F}=\mathbb{F} \cap(\mathbb{P} \times G)$.

\subsubsection{Real projective case.}

Recall that $\mathrm{P}$ denotes a real projective space of which $\mathbb{P}$ is a complexification, and $\mathcal{C}_{\mathrm{P}}^{\infty}(m \mid \varepsilon)$ is the sheaf of sections of the $C^{\infty}$ line bundle described by the homogeneity condition

$$
\varphi(\lambda x)=(\operatorname{sgn} \lambda)^{\varepsilon} \lambda^{m} \varphi(x) \quad \text { for } \lambda \in \mathbf{R}^{\times} .
$$

Proof of Proposition 1.3.1. Since $\pi_{1}(\mathrm{P})=\mathbf{Z} / 2 \mathbf{Z}$, there are essentially two local systems of rank one on $P$. Let us denote them by $C_{P}^{(\varepsilon)}$, for $\varepsilon \in \mathbb{Z} / 2 \mathbb{Z}$, so that $\mathbf{C}_{\mathrm{P}}^{(0)}$ is the constant sheaf $\mathbf{C}_{\mathrm{P}}$. Then, one checks that

$$
\mathcal{C}^{\natural}\left(\mathbf{C}_{\mathrm{P}}^{(\varepsilon)}\right) \simeq \mathcal{C}_{\mathrm{P}}^{\natural}(0 \mid \varepsilon),
$$

for $\natural= \pm \infty, \pm \omega$. Similarly, recall that $\pi_{1}(G)=\mathbf{Z} / 2 \mathbb{Z}$, and note that

$$
\mathcal{C}^{\natural}\left(\mathbf{C}_{\mathrm{G}}^{(\varepsilon)}\right) \simeq \mathcal{C}_{\mathrm{G}}^{\natural}(0 \mid \varepsilon)
$$

By the above remarks, the statement of the proposition can be rewritten as

$$
H^{0}\left(\mathbb{P} ; \mathcal{C}^{\infty}\left(\mathbb{C}_{\mathrm{P}}^{(-p-1)}\right)\left(m^{*}\right)\right) \stackrel{\sim}{\longrightarrow} H^{0} \operatorname{Sol}\left(\square_{m}, \mathcal{C}^{\infty}\left(\mathbf{C}_{\mathrm{G}}^{(-1)}\right)\right)
$$

By Proposition 3.3.1 below, this is a corollary of Corollary 2.4.3, with $\mathcal{G}=$ $\mathrm{C}_{\mathrm{G}}^{(-1)}$, and $\mathcal{F}=\mathbb{C}_{\mathrm{P}}^{(-p-1)}$.

Proposition 3.3.1. With the above notations, one has

$$
\tau \leq p\left(\mathbf{C}_{\mathbb{F}} \circ \mathbf{C}_{\mathbf{G}}^{(-1)}\right) \simeq \begin{cases}\mathbf{C}_{\mathbb{P} \backslash \mathrm{P}[-p+1]} & \text { for } p \text { odd } \\ \mathbf{C}_{\mathbf{P}}^{(1)}[-p] & \text { for } p \text { even } .\end{cases}
$$

In particular,

$$
\tau^{\leq p}\left(\mathbf{C}_{\mathbb{F}} \circ \mathbf{C}_{\mathrm{G}}^{(-1)}\right) \doteq \mathbf{C}_{\mathrm{P}}^{(-p-1)}[-p]
$$


Proof. The second assertion follows from the first one by considering the distinguished triangle associated with the short exact sequence

$$
0 \rightarrow \mathbf{C}_{\mathbb{P} \backslash \mathrm{P}} \rightarrow \mathbf{C}_{\mathbb{P}} \rightarrow \mathbf{C}_{\mathrm{P}} \rightarrow 0 .
$$

Let us prove the first assertion. By (2.2.5), one has

$$
\mathbf{C}_{\mathbb{F}} \circ \mathbf{C}_{\mathrm{G}}^{(\varepsilon)} \simeq R f_{!} \mathbf{C}_{\widetilde{\mathrm{F}}}^{(\varepsilon)}
$$

where $\mathbf{C}_{\widetilde{F}}^{(\varepsilon)}=\mathbf{C}_{\widetilde{F}} \otimes g^{-1} \mathbf{C}_{\mathrm{G}}^{(\varepsilon)}$. The natural identification $\mathrm{V} \oplus i \mathrm{~V} \stackrel{\sim}{\longrightarrow} \vee \otimes_{\mathbf{R}} \mathbf{C}=\mathbb{V}$ induces a projection $\mathbb{V} \rightarrow \mathrm{V}$, which in turn induces a map

$$
\rho: \mathbb{P} \rightarrow \mathrm{P} \sqcup \mathrm{Gr}(2 ; \mathrm{V}) .
$$

More precisely, for $x, y \in \mathrm{V}$ one has $\rho\left(\langle x+i y\rangle_{\mathbf{C}}\right)=\langle x, y\rangle_{\mathbf{R}}$, so that $\left.\rho\right|_{\mathrm{P}}$ is the identity, and $\rho^{-1}(\eta)=\operatorname{Gr}\left(1 ; \eta \otimes_{\mathbf{R}} \mathbf{C}\right)$ for $\eta \in \operatorname{Gr}(2 ; \mathrm{V})$. For $z \in \mathbb{P}, \xi \in \mathrm{G}$, one has

$$
(z, \xi) \in \widetilde{\mathrm{F}} \Longleftrightarrow z \subset \xi \otimes_{\mathbf{R}} \mathbf{C} \Longleftrightarrow \rho(z) \subset \xi,
$$

and hence the fiber $\widetilde{\mathrm{F}}_{z}=\widetilde{\mathrm{F}} \cap f^{-1}(z)$ has the description

$$
\widetilde{\mathrm{F}}_{z}=\{\xi \in \mathrm{G}: \rho(z) \subset \xi\} \simeq \begin{cases}\operatorname{Gr}(p ; \mathrm{V} / \rho(z)) & \text { if } z \in \mathrm{P} \\ \operatorname{Gr}(p-1 ; \mathrm{V} / \rho(z)) & \text { if } z \in \mathbb{P} \backslash \mathrm{P} .\end{cases}
$$

Applying the functor $R f_{!}$to the short exact sequence

$$
0 \rightarrow \mathbf{C}_{\widetilde{F} \backslash \mathrm{F}}^{(-1)} \rightarrow \mathbf{C}_{\widetilde{F}}^{(-1)} \rightarrow \mathbf{C}_{\mathrm{F}}^{(-1)} \rightarrow 0
$$

we get the distinguished triangle

$$
R f_{!} \mathbf{C}_{\widetilde{\mathrm{F}} \backslash \mathrm{F}}^{(-1)} \rightarrow \mathbf{C}_{\mathbb{F}} \circ \mathbf{C}_{\mathrm{G}}^{(-1)} \rightarrow R f_{!} \mathbf{C}_{\mathrm{F}}^{(-1)} \underset{+1}{\longrightarrow}
$$

By (3.3.1), $\widetilde{\mathrm{F}} \backslash \mathrm{F}$ is a $\operatorname{Gr}\left(p-1 ; \mathbf{R}^{n-1}\right)$-bundle over $\mathbb{P} \backslash \mathrm{P}$, which is simply connected, and hence

$$
H^{k} R f_{!} \mathbf{C}_{\widetilde{\mathrm{F}} \backslash \mathrm{F}}^{(-1)} \simeq P_{\mathbb{P} \backslash \mathrm{P}}^{k}, \quad \text { for } P^{k}=H^{k}\left(\mathrm{Gr}\left(p-1 ; \mathbf{R}^{n-1}\right) ; \mathbf{C}_{\mathrm{Gr}\left(p-1 ; \mathbf{R}^{n-1}\right)}^{(-1)}\right) .
$$

Moreover, $\mathrm{F}$ is a $\operatorname{Gr}\left(p ; \mathbf{R}^{n}\right)$-bundle over $\mathrm{P}$, which is not simply connected, and hence

$$
H^{k} R f_{!} \mathbf{C}_{\mathrm{F}}^{(-1)} \text { is a locally free sheaf on } \mathrm{P} \text { of } \operatorname{rank} b_{(-1)}(k ; p, n),
$$

where $b_{(-1)}(k ; p, n)=\operatorname{dim} H^{k}\left(\operatorname{Gr}\left(p ; \mathbf{R}^{n}\right) ; \mathbf{C}_{\operatorname{Gr}\left(p ; \mathbf{R}^{n}\right)}^{(-1)}\right)$. 
Let us consider the case $p+1 \leq n-p$. Concerning the topology of $\operatorname{Gr}\left(q ; \mathbb{R}^{m}\right)$, we need the following result, valid for $1 \leq q \leq m-q$

$$
H^{k}\left(\operatorname{Gr}\left(q ; \mathbb{R}^{m}\right) ; \mathbf{C}_{\mathrm{Gr}\left(q ; \mathbf{R}^{m}\right)}^{(-1)}\right)= \begin{cases}0 & k<q \\ 0 & k=q \text { and } q \text { odd } \\ \mathbf{C} & k=q \text { and } q \text { even }\end{cases}
$$

(This is a particular case of Proposition 3.6.3 (ii).) Using (3.3.3), (3.3.4), and (3.3.5), we can compute the beginning of the long exact cohomology sequence associated with (3.3.2). This gives

$$
\tau \leq p\left(\mathbf{C}_{\mathbb{F}} \circ \mathbf{C}_{\mathrm{G}}^{(-1)}\right) \simeq \begin{cases}\mathbf{C}_{\mathbb{P} \backslash \mathrm{P}[-p+1]} & \text { for } p \text { odd } \\ \mathbf{C}_{\mathrm{P}}^{(\varepsilon)}[-p] & \text { for } p \text { even }\end{cases}
$$

and we are left to check that $\varepsilon \equiv-p-1 \equiv 1 \bmod 2$. This follows from

$$
\begin{aligned}
\Gamma\left(\mathrm{P} ; \mathbf{C}_{\mathrm{P}}^{(\varepsilon)}\right) & \simeq \operatorname{Hom}\left(\mathbf{C}_{\mathbb{P}}, \mathbf{C}_{\mathrm{P}}^{(\varepsilon)}\right) \\
& \simeq \operatorname{Hom}\left(\mathbf{C}_{\mathbb{P}}, \mathbf{C}_{\mathbb{F}} \circ \mathbf{C}_{\mathrm{G}}^{(-1)}[p]\right) \\
& \simeq \operatorname{Hom}\left(\mathbf{C}_{\mathbb{P}} \circ \mathbf{C}_{\mathbb{F}}, \mathbf{C}_{\mathrm{G}}^{(-1)}[-p]\right) \\
& \simeq \bigoplus_{k=0}^{p} H^{2 k-p}\left(\mathbb{G} ; \mathbf{C}_{\mathrm{G}}^{(-1)}\right)=0,
\end{aligned}
$$

where the second isomorphism is due to (3.3.6), the third one to (2.2.3), the fourth one to Lemma 3.3.3 below, and the last term vanishes by (3.3.5). This concludes the proof for the case $p+1 \leq n-p$.

Let us now consider the case $p+1>n-p$. As we already noticed, there is a natural identification $\mathbb{G} \simeq \operatorname{Gr}\left(n-p ; \mathbb{V}^{*}\right)$ obtained by mapping a subspace of $\mathbb{V}$ to its orthogonal in $\mathbb{V}^{*}$. Let $\mathbb{P}^{*} \simeq \operatorname{Gr}\left(1 ; \mathbb{V}^{*}\right)$ be the dual projective space, and denote by $\mathbb{F}^{*} \subset \mathbb{P}^{*} \times \mathbb{G}$ the incidence relation. As in the Appendix, denote by $\mathbb{S} \subset \mathbb{P} \times \mathbb{P}^{*}$ the incidence relation associated with the projective duality, and consider the correspondences

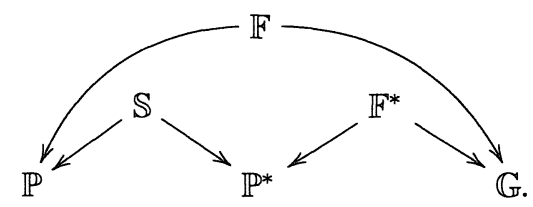

Set for short

$$
\mathbb{S}^{c}=\left(\mathbb{P} \times \mathbb{P}^{*}\right) \backslash \mathbb{S}, \quad \mathbb{F}^{c}=(\mathbb{P} \times \mathbb{G}) \backslash \mathbb{F}
$$


The statement follows from the chain of isomorphisms

$$
\begin{aligned}
\tau^{\leq p}\left(\mathbf{C}_{\mathbb{F}} \circ \mathbf{C}_{\mathrm{G}}^{(-1)}\right) & \doteq \tau^{\leq p}\left(\mathbf{C}_{\mathbb{S}^{c}} \circ \mathbf{C}_{\mathbb{F}^{*}} \circ \mathbf{C}_{\mathrm{G}}^{(-1)}{ }_{[2 n-2 p-1]}\right) \\
& =\tau^{\leq n-p-1}\left(\mathbf{C}_{\mathbb{S}^{c}} \circ \mathbf{C}_{\mathbb{F}^{*}} \circ \mathbf{C}_{\mathrm{G}}^{(-1)}\right)[2 n-2 p-1] \\
& \simeq \tau^{\leq n-p-1}\left(\mathbf{C}_{\mathbb{S}^{c}} \circ \mathbf{C}_{\mathbf{P}^{*}}^{(p-n)}[-(n-p-1)]\right)[2 n-2 p-1] \\
& \simeq \tau^{\leq 0}\left(\mathbf{C}_{\mathbb{S}^{c}} \circ \mathbf{C}_{\mathbf{P}^{*}}^{(p-n)}\right)[n-p] \\
& \doteq \mathbf{C}_{\mathrm{P}}^{(-p-1)}[-p] .
\end{aligned}
$$

Here, the first equality follows from Lemma 3.3.2 below, the second and fourth ones from the identity $\tau^{\leq a}(X[b])=\tau^{\leq a+b} X[b]$, and the fifth one from (A.2.1). We are then reduced to prove the third isomorphism. Since the roles of $p+1$ and $n-p$ are interchanged when considering the incidence relation $\mathbb{F}^{*}$ instead of $\mathbb{F}$, the first part of the proof gives

$$
\tau^{\leq n-p-1}\left(\mathbf{C}_{\mathbb{F}^{+}} \circ \mathbf{C}_{\mathrm{G}}^{(-1)}\right) \doteq \mathbf{C}_{\mathbf{P}^{*}}^{(p-n)}[-(n-p-1)] .
$$

It is then possible to apply Lemma 2.4.2 for $\mathcal{C}=\operatorname{Mod}\left(\mathbf{C}_{\mathbb{P}^{*}}\right), \mathcal{C}^{\prime}=\operatorname{Mod}\left(\mathbf{C}_{\mathbb{P}}\right)$, $\Phi=\mathbf{C}_{\mathbb{S}^{c}[n]} \circ(\cdot), \mathcal{N}$ the null system of constant objects, $X=\mathbf{C}_{\mathbb{F}^{*}} \circ \mathbf{C}_{\mathrm{G}}^{(-1)}$, $Y=\mathbf{C}_{\mathrm{P}^{*}}^{(p-n)}[-(n-p-1)], r=n-p-1$, and $s=n$. This gives

$$
\tau^{\leq n-p-1}\left(\mathbf{C}_{\mathbb{S}^{c}} \circ \mathbf{C}_{\mathbb{F}^{*}} \circ \mathbf{C}_{\mathrm{G}}^{(-1)}\right) \simeq \tau^{\leq n-p-1}\left(\mathbf{C}_{\mathbb{S}^{c}} \circ \mathbf{C}_{\mathbf{P}^{*}}^{(p-n)}[-(n-p-1)]\right) .
$$

Lemma 3.3.2. With the above notations, one has

$$
\mathbf{C}_{\mathbb{S}^{c}} \circ \mathbf{C}_{\mathbb{F}^{*}} \simeq \mathbf{C}_{\mathbb{F}^{c}[-2(n-p-1)]},
$$

and hence

$$
\mathbf{C}_{\mathbb{F}} \doteq \mathbf{C}_{\mathbb{S}^{c}} \circ \mathbf{C}_{\mathbb{F}^{*}}[2(n-p)-1]
$$

Proof. The stalk of $\mathbf{C}_{\mathbb{S}^{c}} \circ \mathbf{C}_{\mathbb{F}^{*}}$ at the point $(z, \zeta) \in \mathbb{P} \times \mathbb{G}$ is described by the compactly supported cohomology of the set

$$
\left(\{z\} \times \mathbb{P}^{*} \times\{\zeta\}\right) \cap\left(\mathbb{S}^{c} \times \mathbb{P}^{*} \mathbb{F}^{*}\right)=\left\{\eta \in \mathbb{P}^{*}: z \not \subset \eta, \zeta \subset \eta\right\} .
$$

Such a set is empty if $(z, \zeta) \in \mathbb{F}$, and isomorphic to $\mathbf{C}^{n-p-1}$ otherwise. Since $\mathbb{F}^{c}$ is simply connected, the locally constant sheaf $\left.\left(\mathbf{C}_{\mathbb{S}^{c}} \circ \mathbf{C}_{\mathbb{F}^{*}}[-2(n-p-1)]\right)\right|_{\mathbb{F}^{c}}$ is constant, and the lemma is proved.

Finally, let us prove the following lemma, that we used in the first part of the proof of Proposition 3.3.1. 
Lemma 3.3.3. One has

$$
\mathbf{C}_{\mathbb{P}} \circ \mathbf{C}_{\mathbb{F}} \simeq \bigoplus_{k=0}^{p} \mathbf{C}_{\mathbb{G}[-2 k]}
$$

Proof. Since $\mathbf{C}_{\mathbb{P}} \circ \mathbf{C}_{\mathbb{F}} \simeq R g_{!} \mathbf{C}_{\mathbb{F}}$, the stalk at $\zeta \in \mathbb{G}$ is computed by

$$
\left(\mathbf{C}_{\mathbb{P}} \circ \mathbf{C}_{\mathbb{F}}\right)_{\zeta} \simeq \mathrm{R} \Gamma_{c}\left(\mathbb{F}_{\zeta} ; \mathbf{C}\right)
$$

where

$$
\mathbb{F}_{\zeta}=g^{-1}(\zeta)=\{z \in \mathbb{P}: z \subset \zeta\}=\operatorname{Gr}(1 ; \zeta)
$$

is a complex $p$-dimensional projective space. In particular, $\mathbf{C}_{\mathbb{P}} \circ \mathbf{C}_{\mathbb{F}}$ is locally constant. Moreover, since $\mathbb{G}$ is simply connected, one has

$$
H^{k}\left(\mathbf{C}_{\mathbb{P}} \circ \mathbf{C}_{\mathbb{F}}\right) \simeq \begin{cases}\mathbf{C}_{\mathbb{G}} & \text { for } k=2 j, 0 \leq j \leq p, \\ 0 & \text { otherwise }\end{cases}
$$

where we used the well-known relation

$$
H^{k}\left(\operatorname{Gr}\left(1 ; \mathbf{C}^{p+1}\right) ; \mathbf{C}_{\mathrm{Gr}\left(1 ; \mathbf{C}^{p+1}\right)}\right)= \begin{cases}\mathbf{C} & \text { for } k=2 j, 0 \leq j \leq p, \\ 0 & \text { otherwise }\end{cases}
$$

We are thus reduced to check that this complex splits as the direct sum of its cohomology groups, which is true since cohomology occurs only in even degrees.

Remark. For $z \in \mathbb{P}$, one has $\mathbb{F}_{z}=\{\zeta \in \mathbb{G}: \zeta \supset z\} \simeq \operatorname{Gr}(p ; \mathbb{V} / \zeta)$. Then, using Proposition 3.7.2, one can similarly prove the isomorphism

$$
\mathbf{C}_{\mathbb{F}} \circ \mathbf{C}_{\mathbb{G}} \simeq \bigoplus_{k=0}^{p(n-p)} \mathbf{C}_{\mathbb{P}}^{\left.d(k ; p, n)_{\mathrm{r}}-2 k\right]}
$$

\subsubsection{Real affine case.}

As in section 1.3.2, let $H \subset P$ be a hyperplane, and set $A=P \backslash H, G_{A}=$ $\mathrm{G} \backslash \mathrm{G}_{\mathrm{H}}$, where $\mathrm{G}_{\mathrm{H}}$ is the Grassmann manifold of projective $p$-dimensional planes in $\mathrm{H}$.

Proof of Proposition 1.3.2. Since $A$ is open subanalytic in $P, \mathcal{C}^{\infty}\left(\mathbf{C}_{A}\right)=$ $\mathcal{I}_{\mathrm{H} \mid \mathrm{P}}^{\infty}$ is the ideal of $\mathcal{C}_{\mathrm{P}}^{\infty}$ of functions vanishing up to infinite order on $\mathrm{H}$. In particular,

$$
\begin{aligned}
\mathcal{S}(\mathrm{A}) & \simeq H^{0}\left(\mathbb{P} ; \mathcal{C}^{\infty}\left(\mathbf{C}_{\mathrm{A}}\right)\right) \\
& \simeq H^{0}\left(\mathbb{P} ; \mathcal{C}^{\infty}\left(\mathbf{C}_{\mathbf{A}}^{(-p-1)}\right)\left(m^{*}\right)\right)
\end{aligned}
$$


where the last isomorphism is due to the fact that $A$ is an affine chart in $P$. Applying the functor $\mathrm{R} \Gamma\left(\mathbb{P} ; \mathcal{C}^{\infty}(\cdot)\left(m^{*}\right)\right)$ to the short exact sequence

$$
0 \rightarrow \mathbf{C}_{\mathrm{A}}^{(-p-1)} \rightarrow \mathbf{C}_{\mathrm{P}}^{(-p-1)} \rightarrow \mathbf{C}_{\mathbf{H}}^{(-p-1)} \rightarrow 0
$$

we then get

$$
\mathcal{S}(\mathrm{A}) \simeq \operatorname{ker}\left(H^{0}\left(\mathbb{P} ; \mathcal{C}^{\infty}\left(\mathbf{C}_{\mathrm{P}}^{(-p-1)}\right)\left(m^{*}\right)\right) \rightarrow H^{0}\left(\mathbb{P} ; \mathcal{C}^{\infty}\left(\mathbf{C}_{\mathrm{H}}^{(-p-1)}\right)\left(m^{*}\right)\right)\right)
$$

Similarly, $\left\{\psi \in \mathcal{S}_{\left(H_{m} \mid-1\right)}\left(\mathrm{G}_{\mathrm{A}}\right): \square_{m} \psi=0\right\}$ is isomorphic to

$$
\operatorname{ker}\left(H^{0} \operatorname{Sol}\left(\square_{m}, \mathcal{C}^{\infty}\left(\mathbf{C}_{\mathrm{G}}^{(-1)}\right)\right) \rightarrow H^{0} \operatorname{Sol}\left(\square_{m}, \mathcal{C}^{\infty}\left(\mathbf{C}_{\mathrm{G}_{\mathrm{H}}}^{(-1)}\right)\right)\right) .
$$

By Proposition 1.3.1, we know that

$$
H^{0}\left(\mathbb{P} ; \mathcal{C}^{\infty}\left(\mathbf{C}_{\mathrm{P}}^{(-p-1)}\right)\left(m^{*}\right)\right) \stackrel{\sim}{\rightarrow} H^{0} \operatorname{Sol}\left(\square_{m}, \mathcal{C}^{\infty}\left(\mathbf{C}_{\mathrm{G}}^{(-1)}\right)\right)
$$

It is then enough to prove the isomorphism

$$
H^{0}\left(\mathbb{P} ; \mathcal{C}^{\infty}\left(\mathbf{C}_{\mathrm{H}}^{(-p-1)}\right)\left(m^{*}\right)\right) \stackrel{\sim}{\longrightarrow} H^{0} \operatorname{Sol}\left(\square_{m}, \mathcal{C}^{\infty}\left(\mathbf{C}_{\mathrm{G}_{\mathrm{H}}}^{(-1)}\right)\right) .
$$

Denote by $j: \mathbb{H} \hookrightarrow \mathbb{P}$ the complexification of $\mathrm{H} \subset \mathrm{P}$. Let $\mathcal{F}$ be as in Proposition 3.3.4 below. One has the chain of isomorphisms

$$
\begin{aligned}
H^{0}\left(\mathbb{P} ; \mathcal{C}^{\infty}\left(\mathbf{C}_{\mathrm{H}}^{(-p-1)}\right)\left(m^{*}\right)\right) & \simeq H^{p}\left(\mathbb{P} ; \mathcal{C}^{\infty}(j ! \mathcal{F})\left(m^{*}\right)\right) \\
& \simeq H^{0} \operatorname{Sol}\left(\square_{m}, \mathcal{C}^{\infty}\left(\mathbf{C}_{\mathrm{G}_{\mathrm{H}}}^{(-1)}\right)\right)
\end{aligned}
$$

where the first one is due to Lemma 2.4 .2 for $\Phi=\mathrm{R} \Gamma\left(\mathbb{P} ; \mathcal{C}^{\infty}\left(j_{!}(\cdot)\right)\left(m^{*}\right)\right)$, hypothesis (a) being implied by Lemma 3.3.5 below, and the second one follows from Corollary 2.4.3.

Proposition 3.3.4. Denoting by $j: \mathbb{H} \hookrightarrow \mathbb{P}$ the complexification of $\mathrm{H} \subset$ $\mathrm{P}$, one has

$$
\tau^{\leq p}\left(\mathbf{C}_{\mathbb{F}} \circ \mathbf{C}_{\mathrm{G}_{\mathrm{H}}}^{(1)}\right) \doteq j ! \mathcal{F}
$$

where $\mathcal{F} \doteq \mathbf{C}_{\mathbf{H}}^{(p+1)}[-p]$ in $\mathbf{D}^{\mathrm{b}}\left(\mathbf{C}_{\mathbb{H}} ; \dot{T}^{*} \mathbb{H}\right)$.

Proof. Denote by $k: \mathbb{G}_{\mathbb{H}} \hookrightarrow \mathbb{G}$ the complexification of $\mathrm{G}_{\mathrm{H}} \subset \mathrm{G}$, and set $\mathbb{F}_{\mathbb{H}}=\mathbb{F} \cap\left(\mathbb{H} \times \mathbb{G}_{\mathbb{H}}\right)$. Consider the double fibration

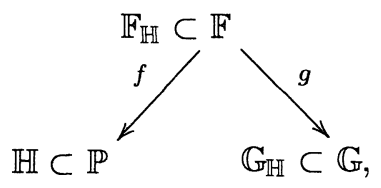


and set $\mathbb{F}^{c}=(\mathbb{P} \times \mathbb{G}) \backslash \mathbb{F}, \mathbb{F}_{\mathbb{H}}^{c}=\left(\mathbb{H} \times \mathbb{G}_{\mathbb{H}}\right) \backslash \mathbb{F}_{\mathbb{H}}$. As pointed out in [3, Lemma 3.3], one has $\mathbf{C}_{\mathbb{F}^{c}} \circ k_{!}(\cdot) \simeq j_{!}\left(\mathbf{C}_{\mathbb{F}_{\mathbb{H}}} \circ \cdot\right)$. Then

$$
\begin{aligned}
& \tau^{\leq p}\left(\mathbf{C}_{\mathbb{F}} \circ \mathbf{C}_{\mathrm{G}_{\mathrm{H}}}^{(1)}\right)=\tau^{\leq p}\left(\mathbf{C}_{\mathbb{F}} \circ k_{!} \mathbf{C}_{\mathrm{G}_{\mathrm{H}} \mid \mathbb{G}_{\mathbb{H}}}^{(1)}\right) \\
& \doteq \tau^{\leq p}\left(\mathbf{C}_{\mathbb{F}^{c}} \circ k_{!} \mathbf{C}_{\mathbf{G}_{\mathrm{H}} \mid \mathbb{G}_{\mathbb{H}}}^{(1)}\right) \\
& \simeq \tau^{\leq p} j_{!}\left(\mathbb{C}_{\mathbb{F}_{\mathbb{H}} c} \circ \mathbf{C}_{\mathrm{G}_{\mathrm{H}} \mid \mathbb{G}_{\mathbb{H}}}^{(1)}\right) \\
& \simeq j_{!} \tau^{\leq p}\left(\mathbf{C}_{\mathbb{F}_{\mathbb{H}}} \circ \mathbf{C}_{\mathbf{G}_{\mathrm{H}} \mid \mathbb{G}_{\mathbb{H}}}^{(1)}\right),
\end{aligned}
$$

where the fourth isomorphism holds since $j$ ! is an exact functor. Finally, one has

$$
\begin{aligned}
\mathcal{F} & =\tau^{\leq p}\left(\mathbf{C}_{\mathbb{F}_{\mathbb{H}} c} \circ \mathbf{C}_{\mathrm{G}_{H} \mid \mathbb{G}_{\mathbb{H}}}^{(1)}\right) \\
& \doteq \tau^{\leq p}\left(\mathbf{C}_{\mathbb{F}_{\mathbb{H}}} \circ \mathbf{C}_{\mathrm{G}_{H} \mid \mathbb{G}_{H}}^{(1)}\right) \\
& \doteq \mathbb{C}_{\mathrm{H} \mid \mathbb{H}}^{(p+1)}[-p]
\end{aligned}
$$

in $\mathbf{D}^{\mathrm{b}}\left(\mathbf{C}_{\mathbb{H}} ; \dot{T}^{*} \mathbb{H}\right)$, where the second isomorphism is due to Proposition 3.3.1.

Lemma 3.3.5. For $m^{*}<0$ the complex $\mathrm{R} \Gamma\left(\mathbb{P} ; \mathcal{C}^{\infty}\left(\mathbf{C}_{\mathbb{H}}\right)\left(m^{*}\right)\right)$ is concentrated in degree $n-1$. In particular, if $N \in \mathbf{D}^{\geq 0}\left(\mathbf{C}_{\mathbb{H}}\right)$ is zero in $\mathbf{D}^{\geq 0}\left(\mathbb{C}_{\mathbb{H}} ; \dot{T}^{*} \mathbb{H}\right)$ (i.e. $N \doteq 0)$, then $\tau^{<n-1}\left(\mathrm{R} \Gamma\left(\mathbb{P} ; \mathcal{C}^{\infty}(N)\left(m^{*}\right)\right)\right)=0$.

Proof. Since $\mathbb{H}$ is simply connected, $H^{j}(N)$ is a constant sheaf along $\mathbb{H}$. It is then enough to show that $\mathrm{R} \Gamma\left(\mathbb{P} ; \mathcal{C}^{\infty}\left(\mathbf{C}_{\mathbb{H}}\right)\left(m^{*}\right)\right)$ is in degree $n-1$. As in the Appendix, denote by $\mathbb{P}^{*}$ the dual projective space, by $\mathbb{S}$ the incidence relation, and set $\mathbb{S}^{c}=\left(\mathbb{P} \times \mathbb{P}^{*}\right) \backslash \mathbb{S}$. Let $h \in \mathbb{P}^{*}$ be the point such that $\widehat{h}=\mathbb{H}$. By Theorem A.1.1, one has

$$
\mathrm{R} \Gamma\left(\mathbb{P} ; \mathcal{C}^{\infty}\left(\mathbf{C}_{\mathbb{S}^{c}}^{\prime} \circ \mathbf{C}_{h}\right)\left(m^{*}\right)\right)[n] \simeq \mathcal{O}_{\mathbb{P}^{*}\left(-m^{*}-n-1\right) \widehat{\mid}_{h}}
$$

Note that $\mathbf{C}_{\mathbb{S}} \circ \mathbf{C}_{h} \simeq \mathbf{C}_{\mathbb{H}}, \mathbf{C}_{\mathbb{P} \times \mathbb{P}^{*}} \circ \mathbf{C}_{h} \simeq \mathbf{C}_{\mathbb{P}}$, and that Serre's isomorphism reads

$$
\mathrm{R} \Gamma\left(\mathbb{P} ; \mathcal{O}_{\mathbb{P}}\left(m^{*}\right)\right)[n] \simeq \mathrm{R} \Gamma\left(\mathbb{P}^{*} ; \mathcal{O}_{\mathbb{P}}\left(-m^{*}-n-1\right)\right),
$$

where both complexes are concentrated in degree zero. From the short exact sequence $0 \rightarrow \mathbf{C}_{\mathbb{S}^{c}} \rightarrow \mathbb{C}_{\mathbb{P} \times \mathbb{P}^{*}} \rightarrow \mathbb{C}_{\mathbb{S}} \rightarrow 0$, one gets the distinguished triangle

$$
\mathbf{C}_{\mathbb{P} \times \mathbb{P}^{*}} \rightarrow \mathbf{C}_{\mathbb{S}^{c}}^{\prime} \rightarrow \mathbf{C}_{\mathbb{S}[-1] \underset{+1}{\longrightarrow}}^{\longrightarrow}
$$

Applying the functor $\mathrm{R} \Gamma\left(\mathbb{P} ; \mathcal{C}^{\infty}\left(\cdot \circ \mathbb{C}_{h}\right)\left(m^{*}\right)\right)$, we get the distinguished triangle

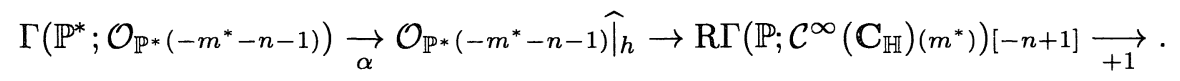

One concludes since $\alpha$ is injective. 


\section{§3.4. Action of $S L(n+1, \mathbf{C})_{\mathbf{R}}$}

\subsubsection{Realified complex projective case.}

Let us use the notations of Section 1.4.1. In particular, $\mathbb{P}_{\mathbf{R}}$ denotes the real analytic manifold underlying $\mathbb{P}$, and $\mathbb{P} \times \overline{\mathbb{P}} \supset \mathbb{P}_{\mathbf{R}}$ its natural complexification.

Let us denote by $\underset{D}{D}$ the exterior tensor product for $\mathcal{D}$-modules. Using the $\mathcal{D}$-module analogue of formula (2.2.7), one gets the following variation of Corollary 2.4.3.

Corollary 3.4.1. Let $\mathcal{F}$ be an $\mathbf{R}$-constructible sheaf on $\mathbb{P} \times \overline{\mathbb{P}}$, and $\mathcal{G}$ an $\mathbf{R}$-constructible sheaf on $\mathbb{G} \times \overline{\mathbb{G}}$. Assume that $\tau \leq 2 p\left(\mathbf{C}_{\mathbb{F} \times \overline{\mathbb{F}}} \circ \mathcal{G}\right) \doteq \mathcal{F}[-2 p]$. Then, for $m^{*}, \widetilde{m}^{*}<0$ and $\emptyset= \pm \infty, \pm \omega$ one has

$$
H^{0}\left(\mathbb{P} \times \overline{\mathbb{P}} ; \mathcal{C}^{\natural}(\mathcal{F})\left(m^{*}, \widetilde{m}^{*}\right)\right) \simeq H^{0} \operatorname{Sol}\left(\square_{m} \stackrel{D}{\square} \bar{\square}_{\widetilde{m}}, \mathcal{C}^{\natural}(\mathcal{G})\right)
$$

Proof of Proposition 1.4.1. Consider for example the case $\square=\infty$. Since $\mathbb{P} \times \overline{\mathbb{P}}$ is a complexification of $\mathbb{P}_{\mathbf{R}}$, one has $\mathcal{C}^{\infty}\left(\mathbf{C}_{\mathbb{P}_{\mathbf{R}}}\right)=\mathcal{C}_{\mathbb{P}_{\mathbf{R}}}^{\infty}$, and hence

$$
H^{0}\left(\mathbb{P} \times \overline{\mathbb{P}} ; \mathcal{C}^{\infty}\left(\mathbf{C}_{\mathbb{P}_{\mathbf{R}}}\right)\left(m^{*}, \widetilde{m}^{*}\right)\right) \simeq \Gamma\left(\mathbb{P}_{\mathbf{R}} ; \mathcal{C}_{\mathbb{P}_{\mathbf{R}}}^{\infty}\left(m^{*}, \tilde{m}^{*}\right)\right)
$$

Similarly,

$H^{0} \operatorname{Sol}\left(\square_{m} \stackrel{D}{\otimes} \bar{\square}_{\widetilde{m}}, \mathcal{C}^{\infty}\left(\mathbf{C}_{\mathbb{G}_{\mathbf{R}}}\right)\right) \simeq\left\{\psi \in \Gamma\left(\mathbb{G}_{\mathbf{R}} ; \mathcal{C}_{\mathbb{G}_{\mathbf{R}}}^{\infty}\left(H_{m}, \bar{H}_{\widetilde{m}}\right)\right): \square_{m} \psi=\bar{\square}_{\widetilde{m}} \psi=0\right\}$.

By Proposition 3.4.2 below, the statement is then a corollary of Corollary 3.4.1, with $\mathcal{G}=\mathbf{C}_{\mathbb{G}_{\mathbf{R}}}$, and $\mathcal{F}=\mathbf{C}_{\mathbb{P}_{\mathbf{R}}}$.

Proposition 3.4.2. With the above notations, one has

$$
\tau^{\leq 2 p}\left(\mathbf{C}_{\mathbb{F} \times \overline{\mathbb{F}}} \circ \mathbf{C}_{\mathbb{G}_{\mathbf{R}}}\right) \doteq \mathbf{C}_{\mathbb{P}_{\mathbf{R}}}[-2 p] .
$$

Proof. Consider the projections

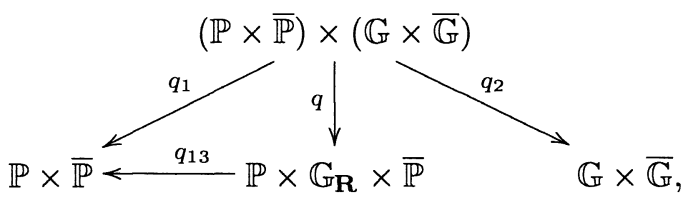

where $q$ is induced by the first projection $\mathbb{G} \times \overline{\mathbb{G}} \rightarrow \mathbb{G}_{\mathbf{R}}$. Setting

$$
\widetilde{\mathbb{F}}=(\mathbb{F} \times \overline{\mathbb{P}}) \cap(\mathbb{P} \times \overline{\mathbb{F}})=\left\{(z, \zeta, w) \in \mathbb{P} \times \mathbb{G}_{\mathbf{R}} \times \overline{\mathbb{P}}: \zeta \supset z+w\right\},
$$


one has

$$
\begin{aligned}
\mathbf{C}_{\mathbb{F} \times \overline{\mathbb{F}}} \circ \mathbf{C}_{\mathbb{G}_{\mathbf{R}}} & \simeq R q_{1 !}\left(\mathbf{C}_{\mathbb{F} \times \overline{\mathbb{F}}} \otimes q_{2}^{-1} \mathbf{C}_{\mathbb{G}_{\mathbf{R}}}\right) \\
& \simeq R q_{13 !} R q_{!} \mathbf{C}_{(\mathbb{F} \times \overline{\mathbb{F}}) \cap q_{2}^{-1}\left(\mathbb{G}_{\mathbf{R}}\right)} \\
& \simeq R q_{13 !} \mathbf{C}_{\widetilde{\mathbb{F}}} .
\end{aligned}
$$

For $(z, w) \in \mathbb{P} \times \overline{\mathbb{P}}$, set $\widetilde{\mathbb{F}}_{(z, w)}=\widetilde{\mathbb{F}} \cap q_{13}^{-1}(z, w)$, and note that

$$
\widetilde{\mathbb{F}}_{(z, w)} \simeq \begin{cases}\operatorname{Gr}(p ; \mathbb{V} / z) & \text { for } z=w \\ \operatorname{Gr}(p-1 ; \mathbb{V} /(z+w)) & \text { for } z \neq w .\end{cases}
$$

Consider the subsets of $\mathbb{P} \times \mathbb{G}_{\mathbf{R}} \times \overline{\mathbb{P}}$

$$
\begin{aligned}
\mathbb{T} & =\left\{(z, \zeta, w) \in \mathbb{P} \times \mathbb{G}_{\mathbf{R}} \times \overline{\mathbb{P}}: \zeta \supset z\right\}, \\
\mathbb{U} & =\mathbb{T} \backslash \widetilde{\mathbb{F}}
\end{aligned}
$$

Applying the functor $R q_{13 \text { ! }}$ to the short exact sequence

$$
0 \rightarrow \mathbf{C}_{\mathbb{U}} \rightarrow \mathbf{C}_{\mathbb{T}} \rightarrow \mathbf{C}_{\widetilde{\mathbb{F}}} \rightarrow 0,
$$

we get a distinguished triangle

$$
R q_{13 !} \mathbf{C}_{\mathbb{U}} \rightarrow R q_{13 !} \mathbf{C}_{\mathbb{T}} \rightarrow \mathbf{C}_{\mathbb{F} \times \overline{\mathbb{F}}} \circ \mathbf{C}_{\mathbb{G}_{\mathbf{R}}} \underset{+1}{\longrightarrow}
$$

Note that $\mathbb{T}_{(z, w)} \simeq \operatorname{Gr}(p ; \mathbb{V} / z)$. Since $\mathbb{T}$ is a $\operatorname{Gr}\left(p ; \mathbf{C}^{n}\right)$-bundle over $\mathbb{P} \times \overline{\mathbb{P}}$, which is simply connected, one has

$$
H^{k}\left(R q_{13 !} \mathbf{C}_{\mathbb{T}}\right) \simeq M_{\mathbb{P} \times \bar{P}}^{k} \quad \text { for } M^{k}=H^{k}\left(\operatorname{Gr}\left(p ; \mathbb{C}^{n}\right) ; \mathbf{C}_{\mathrm{Gr}\left(p ; \mathbf{C}^{n}\right)}\right) .
$$

The fiber $\mathbb{U}_{(z, z)}$ is empty, while $\mathbb{U}_{(z, w)}=\left\{\zeta \in \mathbb{G}_{\mathbf{R}}: \zeta \supset z, \zeta \not \supset w\right\}$ for $z \neq w$. Thus, for $z \neq w$ the projection of the $(p+1)$-dimensional plane $\zeta \in \mathbb{U}_{(z, w)}$ to $\mathbb{V} /(z+w)$ is a $p$-dimensional complex plane. One then has a commutative diagram

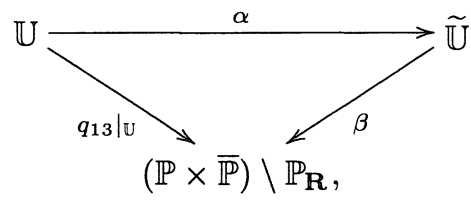

where $\widetilde{\mathbb{U}}$ is the $\operatorname{Gr}\left(p ; \mathbb{C}^{n-1}\right)$-bundle over $(\mathbb{P} \times \overline{\mathbb{P}}) \backslash \mathbb{P}_{\mathbf{R}}$ defined by

$$
\widetilde{\mathbb{U}}_{(z, w)}=\operatorname{Gr}(p ; \mathbb{V} /(z+w)),
$$

and $\alpha, \beta$ are the natural projections. For $z \neq w$ and $\zeta^{\prime} \in \operatorname{Gr}(p ; \mathbb{V} /(z+w))$, one has

$$
\alpha^{-1}\left(z, \zeta^{\prime}, w\right)=\left\{\zeta \in \mathbb{G}_{\mathbf{R}}: \zeta \subset \zeta^{\prime}+z+w, \zeta \supset z\right\} \backslash\{\zeta: \zeta \supset w\},
$$


which is identified with an affine chart in the dual projective space $\operatorname{Gr}\left(p ; \zeta^{\prime}+w\right)$. Since $\alpha$ is a $\mathbf{C}^{p}$-bundle, $\beta$ is a $\operatorname{Gr}\left(p ; \mathbf{C}^{n-1}\right)$-bundle, and $\widetilde{\mathbb{U}}$ and $(\mathbb{P} \times \overline{\mathbb{P}}) \backslash \mathbb{P}_{\mathbf{R}}$ are simply connected, one has

$$
\begin{aligned}
H^{k}\left(R q_{13 !} \mathbf{C}_{\mathbb{U}}\right) & \simeq H^{k}\left(R \beta_{!} \mathbf{C}_{\widetilde{\mathbb{U}}}^{[-2 p]}\right) \\
& \simeq N_{(\mathbb{P} \times \overline{\mathbb{P}}) \backslash \mathbb{P}_{\mathbf{R}}}^{k-2 p} \quad \text { for } N^{\ell}=H^{\ell}\left(\operatorname{Gr}\left(p ; \mathbf{C}^{n-1}\right) ; \mathbf{C}_{\operatorname{Gr}\left(p ; \mathbf{C}^{n-1}\right)}\right)
\end{aligned}
$$

Using (3.4.3) and (3.4.4), we can compute the beginning of the long exact cohomology sequence associated with (3.4.2). This gives

$$
\tau^{\leq 2 p-1}\left(\mathbf{C}_{\mathbb{F} \times \overline{\mathbb{F}}} \circ \mathbf{C}_{\mathbb{G}_{\mathbf{R}}}\right) \simeq \tau^{\leq 2 p-1}\left(R q_{13 !} \mathbf{C}_{\mathbb{T}}\right) \doteq 0 .
$$

Moreover, recalling that cohomology of complex Grassmann manifolds occurs only in even degree, we get an exact sequence

$$
0 \rightarrow N_{(\mathbb{P} \times \overline{\mathbb{P}}) \backslash \mathbb{P}_{\mathbf{R}}}^{0} \rightarrow M_{\mathbb{P} \times \overline{\mathbb{P}}}^{2 p} \rightarrow H^{2 p}\left(\mathbf{C}_{\mathbb{F} \times \overline{\mathbb{F}}} \circ \mathbf{C}_{\mathbb{G}_{\mathbf{R}}}\right) \rightarrow 0 .
$$

Since $\operatorname{Gr}\left(p ; \mathbf{C}^{n-1}\right)$ is connected, by (3.4.4) one has $N^{0}=\mathbf{C}$, and hence

$$
H^{2 p}\left(\mathbf{C}_{\mathbb{F} \times \overline{\mathbb{F}}} \circ \mathbf{C}_{\mathbb{G}_{\mathbf{R}}}\right) \doteq \mathbf{C}_{\mathbb{P}_{\mathbf{R}}} .
$$

The statement follows from (3.4.5) and (3.4.6).

\subsubsection{Realified complex affine case.}

Let us use notations (1.4.2), and consider

$$
\mathbb{G}_{\mathbb{H}}=\widehat{\mathbb{H}} \quad \text { the set of } \zeta \in \mathbb{G} \text { with } \widehat{\zeta} \subset \mathbb{H},
$$

so that $\mathbb{G}_{\mathbb{A}}=\mathbb{G} \backslash \mathbb{G}_{\mathbb{H}}$.

Proof of Proposition 1.4.2. By $(2.1 .3), \mathcal{C}^{\infty}\left(\mathbf{C}_{\mathbb{A}}\right)$ is the ideal of $\mathcal{C}_{\mathbb{P}_{\mathbf{R}}}^{\infty}$ of functions vanishing up to infinite order on $\mathbb{H}$. In particular, taking global sections we have

$$
\begin{aligned}
\mathcal{S}\left(\mathbb{A}_{\mathbf{R}}\right) & \simeq H^{0}\left(\mathbb{P} \times \overline{\mathbb{P}} ; \mathcal{C}^{\infty}\left(\mathbf{C}_{\mathbb{A}}\right)\right) \\
& \simeq H^{0}\left(\mathbb{P} \times \overline{\mathbb{P}} ; \mathcal{C}^{\infty}\left(\mathbf{C}_{\mathbb{A}}\right)\left(m^{*} . \widetilde{m}^{*}\right)\right)
\end{aligned}
$$

The argument then proceeds as in the proof of Proposition 1.3.2, considering the short exact sequence

$$
0 \rightarrow \mathbf{C}_{\mathbb{A}} \rightarrow \mathbf{C}_{\mathbb{P}} \rightarrow \mathbf{C}_{\mathbb{H}} \rightarrow 0
$$




\section{§3.5. Action of $S L((n+1) / 2, \mathbf{H})$}

Recall that $S L((n+1) / 2, \mathbf{H})$ acts transitively on the projective space $\mathbb{P}$. It follows that, with the notations of Section 1.5,

$$
H^{j}\left(\mathbf{C}_{\mathbb{F}} \circ \mathbf{C}_{\mathbf{H G}}\right) \simeq \mathbf{C}_{\mathbb{P}}^{\ell_{j}}, \quad \text { with } \ell_{0}=1
$$

Using Theorem 2.4.1 and Lemma 2.4.4 we get the isomorphism

$$
\tau^{\leq n-p} \operatorname{Sol}\left(\square_{m}, \mathcal{B}_{\mathbf{H} \mathbb{G}}\right) \simeq H^{n}\left(\mathbb{P} ; \mathcal{O}_{\mathbb{P}}\left(m^{*}\right)\right)[p-n],
$$

of which Proposition 1.5.1 is a particular case.

\section{§3.6. More on $S L(n+1, \mathbb{R})$}

As we saw in Section 3.3.1, in order to prove Proposition 1.3.1 it was enough to compute the first non-vanishing cohomology group of the complex $\mathbf{C}_{\mathbb{F}} \circ \mathbb{C}_{\mathrm{G}}^{(-1)}$. Of course, computing higher cohomology groups gives further results. Consider for example the classical Penrose case of $p=1, n=3$. Then, (3.3.2) yields a distinguished triangle

$$
\mathbf{C}_{\mathbb{P} \backslash \mathrm{P}} \rightarrow \mathbf{C}_{\mathbb{F}} \circ \mathbf{C}_{\mathrm{G}}^{(-1)} \rightarrow \mathbf{C}_{\mathbf{P}[-2]} \underset{+1}{\longrightarrow}
$$

Let $m^{*}=-m-3<0$. Applying Theorem 2.4.1 and using Serre isomorphim, one gets an exact sequence

$$
0 \rightarrow H^{1} \operatorname{Sol}\left(\square_{m}, \mathcal{C}^{\infty}\left(\mathbf{C}_{\mathrm{G}}^{(-1)}\right)\right) \rightarrow \Gamma\left(\mathrm{P} ; \mathcal{C}_{\mathrm{P}}^{\infty}\left(m^{*} \mid 0\right)\right) \rightarrow \mathrm{C}[z]_{m} \rightarrow 0,
$$

where $\mathbf{C}[z]_{m}$ is the space of homogeneous polynomial of degree $m$. Since $m>$ -3 , one has in particular

$$
H^{1} \operatorname{Sol}\left(\square_{m}, \mathcal{C}^{\infty}\left(\mathbb{C}_{\mathrm{G}}^{(-1)}\right)\right) \simeq \Gamma\left(\mathrm{P} ; \mathcal{C}_{\mathrm{P}}^{\infty}\left(m^{*} \mid 0\right)\right)
$$

for $m=-2,-1$. This result was also obtained in [9, p. 66-67] using the notion of involutive structure.

As another example of application, one has the following result

Proposition 3.6.1. Let $m \in \mathbf{Z}$ be such that $m^{*}<0$. Then, for some $\varepsilon_{1}, \varepsilon_{2}, \varepsilon_{3} \in \mathbf{Z} / 2 \mathbf{Z}$ one has

(i) if $p$ and $n$ are odd, $H^{n-p} \operatorname{Sol}\left(\square_{m}, \mathcal{C}_{\mathbb{G}}^{\infty}\right) \simeq H^{n}\left(\mathbb{P} ; \mathcal{O}_{\mathbb{P}}\left(m^{*}\right)\right)$,

(ii) if $p$ is odd and $n$ is even, $H^{n-p-1} \operatorname{Sol}\left(\square_{m}, \mathcal{C}_{\mathrm{G}}^{\infty}\right) \simeq \Gamma\left(\mathrm{P} ; \mathcal{C}_{\mathrm{P}}^{\infty}\left(m^{*} \mid \varepsilon_{1}\right)\right)$,

(iii) if $p$ is even and $n$ is odd, $H^{p} \operatorname{Sol}\left(\square_{m}, \mathcal{C}_{\mathrm{G}}^{\infty}\right) \simeq \Gamma\left(\mathrm{P} ; \mathcal{C}_{\mathrm{P}}^{\infty}\left(m^{*} \mid \varepsilon_{2}\right)\right)$,

(iv) if $p$ and $n$ are even, $H^{p} \operatorname{Sol}\left(\square_{m}, \mathcal{C}_{\mathrm{G}}^{\infty}\right) \simeq \Gamma\left(\mathrm{P} ; \mathcal{C}_{\mathrm{P}}^{\infty}\left(m^{*} \mid \varepsilon_{3}\right)\right)$, 
and similarly for $\infty$ replaced by $-\infty, \pm \omega$. Moreover, the lower cohomology groups of the solution complex $\operatorname{Sol}\left(\square_{m}, \mathcal{C}_{\mathrm{G}}^{\infty}\right)$ vanish.

As it was the case for Proposition 1.3.1, its proof is based on the computation of the sheaf-theoretical transform $\mathbf{C}_{\mathbb{F}} \circ \mathbf{C}_{G}$. This is performed in Proposition 3.6.2 below, using the table of Betti numbers for oriented and nonoriented real Grassmann manifolds, that we recall in Proposition 3.6.3. As we will see, the use of such table to compute $\mathbf{C}_{\mathbb{F}} \circ \mathbf{C}_{\mathrm{G}}$ is already quite intricate, and we did not go as far as to determine the twists $\varepsilon_{\imath}$ appearing in the statement.

Proposition 3.6.2. Assume $p+1 \leq n-p$. Then, for some $\varepsilon_{1}, \varepsilon_{2}, \varepsilon_{3} \in$ $\mathbf{Z} / 2 \mathbf{Z}$ one has

(i) if $p$ and $n$ are odd, $\mathbf{C}_{\mathbb{F}} \circ \mathbf{C}_{\mathrm{G}} \doteq 0$,

(ii) if $p$ is odd and $n$ is even, $\tau^{\leq n-1}\left(\mathbf{C}_{\mathbb{F}} \circ \mathbf{C}_{\mathrm{G}}\right) \doteq \mathbf{C}_{\mathrm{P}}^{\left(\varepsilon_{1}\right)}{ }_{[-n+1]}$,

(iii) if $p$ is even and $n$ is odd, $\tau^{\leq 2 p}\left(\mathbf{C}_{\mathbb{F}} \circ \mathbf{C}_{\mathrm{G}}\right) \doteq \mathbf{C}_{\mathrm{P}}^{\left(\varepsilon_{2}\right)}[-2 p]$,

(iv) if $p$ and $n$ are even, $\tau^{\leq 2 p}\left(\mathbf{C}_{\mathbb{F}} \circ \mathbf{C}_{\mathrm{G}}\right) \doteq \mathbf{C}_{\mathrm{P}}^{\left(\varepsilon_{3}\right)}{ }_{[-2 p]}$.

Proof. Consider the projections

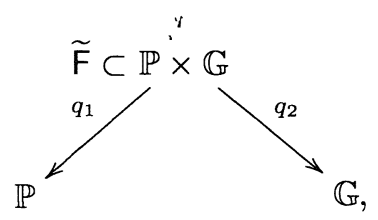

and recall that $\mathbf{C}_{\mathbb{F}} \circ \mathbf{C}_{\mathrm{G}}^{(\varepsilon)} \simeq R q_{1 !} \mathbf{C}_{\widetilde{F}}^{(\varepsilon)}$. In order to argue as in the proof of Proposition 3.4.2, we need to restrict to an affine chart in $\mathbb{P}$. More precisely, let $\left[x_{0}, x^{\prime}\right]=\left[x_{0}, \ldots, x_{n}\right]$ be a system of homogeneous coordinates in $\mathrm{P}$, and denote by $\left[z_{0}, z^{\prime}\right]$ their complexification in $\mathbb{P}$. Denote by $\mathbb{A}^{0}$ the affine chart $z_{0} \neq 0$ in $\mathbb{P}$. Points in $\mathbb{A}^{0}$ can be uniquely written in the form $\left[1, z^{\prime}\right]$, and $\left[1, z^{\prime}\right] \in \mathbb{A}^{0} \cap \mathrm{P}$ if and only if $\left(z^{\prime}\right)$ is real. Writing $z_{j}=x_{j}+i y_{j}$, consider the subsets of $\mathbb{P} \times \mathrm{G}$

$$
\begin{aligned}
\widetilde{\mathrm{F}}^{0} & =\widetilde{\mathrm{F}} \cap\left(\mathbb{A}^{0} \times \mathrm{G}\right)=\left\{\left(\left[1, z^{\prime}\right], \xi\right) \in \mathbb{A}^{0} \times \mathrm{G}: \xi \supset\left[1, x^{\prime}\right]+\left[0, y^{\prime}\right]\right\}, \\
\mathrm{T}^{0} & =\left\{\left(\left[1, z^{\prime}\right], \xi\right) \in \mathbb{A}^{0} \times \mathrm{G}: \xi \supset\left[1, x^{\prime}\right]\right\}, \\
\mathrm{U}^{0} & =\mathrm{T}^{0} \backslash \widetilde{\mathrm{F}}^{0}
\end{aligned}
$$

and note that

$$
\begin{aligned}
& \tilde{\mathrm{F}}_{\left[1, z^{\prime}\right]}^{0} \simeq \begin{cases}\operatorname{Gr}\left(p ; \mathrm{V} /\left[1, x^{\prime}\right]\right) & \text { for }\left[1, z^{\prime}\right] \in \mathbb{A}^{0} \cap \mathrm{P}, \\
\operatorname{Gr}\left(p-1 ; \mathrm{V} /\left(\left[1, x^{\prime}\right]+\left[0, y^{\prime}\right]\right)\right) & \text { for }\left[1, z^{\prime}\right] \in \mathbb{A}^{0} \backslash \mathrm{P},\end{cases} \\
& \mathrm{T}_{\left[1, z^{\prime}\right]}^{0} \simeq \operatorname{Gr}\left(p ; \mathrm{V} /\left[1, x^{\prime}\right]\right) .
\end{aligned}
$$


As in Proposition 3.4.2, one has a commutative diagram

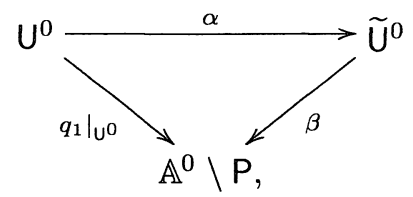

where $\widetilde{U}_{\left[1, z^{\prime}\right]}^{0}=\operatorname{Gr}\left(p ; \mathrm{V} /\left(\left[1, x^{\prime}\right]+\left[0, y^{\prime}\right]\right)\right)$. Of course, similar results hold for any affine chart $\mathbb{A}^{j}=\left\{z_{j} \neq 0\right\}$, giving an open covering of $\mathbb{P}$. In the following, we will denote by $\widetilde{F}^{j}, \mathrm{~T}^{j}, \mathrm{U}^{j}$ the corresponding sets.

Consider the distinguished triangle

$$
\left.R q_{1 !} \mathbf{C}_{\mathrm{U}^{\jmath}}^{(\varepsilon)} \rightarrow R q_{1 !} \mathbf{C}_{\mathrm{T}^{\jmath}}^{(\varepsilon)} \rightarrow\left(\mathbf{C}_{\mathbb{F}} \circ \mathbf{C}_{\mathrm{G}}^{(\varepsilon)}\right)\right|_{\mathbb{A}^{\jmath}} \underset{+1}{\longrightarrow}
$$

Since $\mathrm{T}^{j}$ is a $\operatorname{Gr}\left(p ; \mathbf{R}^{n}\right)$-bundle over $\mathbb{A}^{j}$, which is simply connected, one has

$$
H^{k} R q_{1 !} \mathbf{C}_{\mathrm{T}^{\jmath}}^{(\varepsilon)} \simeq M_{\mathbb{A}^{\jmath}}^{k} \quad \text { for } M^{k}=H^{k}\left(\operatorname{Gr}\left(p ; \mathbf{R}^{n}\right) ; \mathbf{C}_{\mathrm{Gr}\left(p ; \mathbf{R}^{n}\right)}^{(\varepsilon)}\right) .
$$

On the other hand, $\alpha$ is an $\mathbf{R}^{p}$-bundle over $\widetilde{U}^{j}$ which is not simply connected, and $\beta$ is a $\operatorname{Gr}\left(p ; \mathbb{R}^{n-1}\right)$-bundle over $\mathbb{A}^{j} \backslash \mathrm{P}$ which is simply connected. Setting $\bar{\varepsilon} \equiv \varepsilon+1$ we then get

$$
\begin{aligned}
H^{k} R q_{1 !} \mathbf{C}_{\mathrm{U}^{\jmath}}^{(\varepsilon)} & \simeq H^{k}\left(R \beta_{!} \mathbf{C}_{\widetilde{\mathrm{U}}^{\jmath}}^{(\bar{\varepsilon})}[-p]\right) \\
& \simeq N_{\mathbb{A}^{\jmath} \backslash \mathrm{P}}^{k-p} \quad \text { for } N^{\ell}=H^{\ell}\left(\operatorname{Gr}\left(p ; \mathbf{R}^{n-1}\right) ; \mathbf{C}_{\operatorname{Gr}\left(p: \mathbf{R}^{n-1}\right)}^{(\bar{\varepsilon})}\right) .
\end{aligned}
$$

where the first isomorphism comes from Lemma 3.6.4 below, for $\mathrm{W}=\mathrm{V} /\left[1, x^{\prime}\right]$, $y=\left(\left[1, x^{\prime}\right]+\left[0, y^{\prime}\right]\right) /\left[1, x^{\prime}\right]$. Using (3.6.2), (3.6.3), we can compute the beginning of the long exact cohomology sequence associated with (3.6.1). For $\varepsilon \equiv 1$, this would give an alternative proof of Proposition 3.3.1. To prove the present statement, we have to consider the case $\varepsilon \equiv 0$. We shall use Proposition 3.6.3 below.

- If $p$ and $n$ are odd, $N^{\ell}=0$ for any $\ell$, and hence

$$
\mathbf{C}_{\mathbb{F}} \circ \mathbf{C}_{\mathrm{G}} \doteq 0 \text {. }
$$

- If $p$ is odd and $n$ is even, $N^{\ell}=0$ for $\ell \leq n-p-2$. We thus get

$$
\tau^{\leq n-3}\left(\mathbf{C}_{\mathbb{F}} \circ \mathbf{C}_{\mathrm{G}}\right) \doteq 0,
$$

and an exact sequence

$$
\begin{aligned}
0 \rightarrow \mathbf{C}_{\mathbb{A}^{j}}^{d\left(\frac{n-2}{4} ; \frac{n-p-1}{2}, \frac{p-1}{2}\right)} & \left.\rightarrow H^{n-2}\left(\mathbf{C}_{\mathbb{F}} \circ \mathbf{C}_{\mathrm{G}}\right)\right|_{\mathbb{A}^{j}} \rightarrow \mathbf{C}_{\mathbb{A}^{j} \backslash \mathrm{P}} \\
& \left.\rightarrow \mathbf{C}_{\mathbb{A}^{j}} \rightarrow H^{n-1}\left(\mathbf{C}_{\mathbb{F}} \circ \mathbf{C}_{\mathrm{G}}\right)\right|_{\mathbb{A}^{j}} \rightarrow 0 .
\end{aligned}
$$


Since $H^{n-1}\left(\mathbf{C}_{\mathbb{F}} \circ \mathbf{C}_{\mathrm{G}}\right)$ is locally constant of rank one on $\mathrm{P}$, this implies the statement.

- If $p$ is even and $n$ is odd, $N^{\ell}=0$ for $\ell \leq p-1$. We thus get

$$
\tau^{\leq 2 p-2}\left(\mathbf{C}_{\mathbb{F}} \circ \mathbf{C}_{\mathrm{G}}\right) \doteq 0
$$

and an exact sequence

$$
\left.\left.0 \rightarrow H^{2 p-1}\left(\mathbf{C}_{\mathbb{F}} \circ \mathbf{C}_{\mathrm{G}}\right)\right|_{\mathbb{A}^{j}} \rightarrow \mathbf{C}_{\mathbb{A}^{j} \backslash \mathrm{P}} \rightarrow \mathbf{C}_{\mathbb{A}^{j}}^{d\left(\frac{p}{2} ; \frac{n-p-1}{2}, p\right)} \rightarrow H^{2 p}\left(\mathbf{C}_{\mathbb{F}} \circ \mathbf{C}_{\mathrm{G}}\right)\right|_{\mathbb{A}^{j}} \rightarrow 0 .
$$

The computation of the fibers shows that $H^{2 p}\left(\mathbf{C}_{\mathbb{F}} \circ \mathbf{C}_{\mathrm{G}}\right)=0$, and this implies

$$
\tau^{\leq 2 p}\left(\mathbf{C}_{\mathbb{F}} \circ \mathbf{C}_{\mathrm{G}}\right) \doteq \mathbf{C}_{\mathbf{P}}^{\left(\varepsilon_{2}\right)}[-2 p]
$$

for some $\varepsilon_{2} \in \mathbf{Z} / 2 \mathbf{Z}$.

- The case of $p$ and $n$ even is similar to the previous one.

Denote by $\mathrm{Gr}^{+}\left(q ; \mathbf{R}^{m}\right)$ the Grassmannian of real $q$-dimensional oriented subspaces of $\mathbf{R}^{m}$. The projection $\gamma: \operatorname{Gr}^{+}\left(q ; \mathbf{R}^{m}\right) \rightarrow \operatorname{Gr}\left(q ; \mathbf{R}^{m}\right)$ is the universal covering of $\operatorname{Gr}\left(q ; \mathbf{R}^{m}\right)$, and one easily checks that

$$
\gamma_{*} \mathbf{C}_{\mathrm{Gr}^{+}\left(q ; \mathbf{R}^{m}\right)} \simeq \mathbf{C}_{\mathrm{Gr}\left(q ; \mathbf{R}^{m}\right)}^{(0)} \oplus \mathbf{C}_{\mathrm{Gr}\left(q ; \mathbf{R}^{m}\right)}^{(1)} .
$$

The following tables are then immediately derived from Fuks [11].

Proposition 3.6.3. (see e.g. [11]) Consider the partition function

$$
d: \mathbf{Q} \times \mathbf{Z} \times \mathbf{Z} \rightarrow \mathbf{Z}_{\geq 0}
$$

defined as follows. If $k, m, q \in \mathbf{Z}_{>0}$, let $d(k ; m, q)$ be the number of partitions of $k$ in $m$ non-negative integers not bigger than $q$; if $m, q \in \mathbf{Z}_{\geq 0}$ set $d(0 ; m, q)=$ 1 ; set $d(k ; m, q)=0$ otherwise. Then, for $1 \leq q \leq m-1$,

(i) the Betti numbers $\operatorname{dim} H^{k}\left(\operatorname{Gr}\left(q ; \mathbf{R}^{m}\right) ; \mathbf{C}_{\mathrm{Gr}\left(q ; \mathbf{R}^{m}\right)}\right)$ are computed according to

\begin{tabular}{c|c|c|}
\hline$q$ even & $m$ even & $m$ odd \\
\hline \multirow{2}{*}{ odd } & $d\left(\frac{k}{4} ; \frac{m-q}{2}, \frac{q}{2}\right)$ & $d\left(\frac{k}{4} ; \frac{m-q-1}{2}, \frac{q}{2}\right)$ \\
& $d\left(\frac{k-m+1}{4} ; \frac{m-q-1}{2}, \frac{q-1}{2}\right)$ & $d\left(\frac{k-1}{4} ; \frac{m-q}{2}, \frac{q-1}{2}\right)$ \\
\hline
\end{tabular}


(ii) the Betti numbers $\operatorname{dim} H^{k}\left(\operatorname{Gr}\left(q ; \mathbf{R}^{m}\right) ; \mathbf{C}_{\operatorname{Gr}\left(q ; \mathbf{R}^{m}\right)}^{(-1)}\right)$ are computed according to

\begin{tabular}{c|c|c|}
\hline$q$ even & $m$ even & $m$ odd \\
\hline$q\left(\frac{k-m+q}{4} ; \frac{m-q}{2}, \frac{q-2}{2}\right)+$ & $d\left(\frac{k-q}{4} ; \frac{m-q-1}{2}, \frac{q}{2}\right)$ \\
\hline$q$ odd & $d\left(\frac{k-q}{4} ; \frac{m-q-2}{2}, \frac{q}{2}\right)$ & \\
\hline
\end{tabular}

In the proof of Proposition 3.6.2, we used the following generalization of a result used in $[3$, formula (3.14)].

Lemma 3.6.4. Let $y$ be a line in a real vector space W. There is a natural projection

$$
\alpha: \operatorname{Gr}(p ; \mathrm{W}) \backslash\{\eta \in \mathrm{Gr}(p ; \mathrm{W}): \eta \supset y\} \rightarrow \operatorname{Gr}(p ; \mathrm{W} / y)
$$

defined by $\eta \mapsto(\eta+y) / y$, that we denote by $\alpha: \mathrm{G}_{1} \backslash \mathrm{G}_{2} \rightarrow \mathrm{G}_{3}$ for short. Then

$$
R \alpha_{!}\left(\mathbf{C}_{\mathrm{G}_{1} \backslash \mathrm{G}_{2}}^{(\varepsilon)}\right) \simeq \mathbf{C}_{\mathrm{G}_{3}}^{(\bar{\varepsilon})}[-p]
$$

Proof. By the projection formula, $R \alpha_{!}\left(\mathbb{C}_{\mathrm{G}_{1} \backslash \mathrm{G}_{2}}^{(\varepsilon)}\right) \simeq R \alpha_{!}\left(\mathbf{C}_{\mathrm{G}_{1} \backslash \mathrm{G}_{2}}\right) \otimes \mathbf{C}_{\mathrm{G}_{3}}^{(\varepsilon)}$. It is then enough to prove the statement for $\varepsilon \equiv 0$.

The fiber of $\alpha$ at $\xi \in \mathbb{C}_{G_{3}}$ is an affine chart in the dual projective space $\operatorname{Gr}(p ; \xi+y)$. In particular, $\alpha$ is an $\mathbf{R}^{p}$-bundle, and hence there exists $\widetilde{\varepsilon}$ such that

$$
R \alpha ! \mathbf{C}_{\mathrm{G}_{1} \backslash \mathrm{G}_{2}} \simeq \mathbf{C}_{\mathrm{G}_{3}}^{(\widetilde{\varepsilon})}[-p] \text {. }
$$

One may deduce that $\widetilde{\varepsilon} \equiv 1$ from the fact that $\alpha$ is naturally identified with the dual tautological bundle of $\mathrm{G}_{3}$. Alternatively, one may consider the chain of isomorphisms

$$
\begin{aligned}
\Gamma\left(\mathrm{G}_{3} ; \mathbf{C}_{\mathrm{G}_{3}}^{(\widetilde{\varepsilon})}\right) & \simeq \operatorname{Hom}\left(\mathbf{C}_{\mathrm{G}_{3}}^{(\widetilde{\varepsilon})}[-p], \mathbf{C}_{\mathrm{G}_{3}[-p]}\right) \\
& \simeq \operatorname{Hom}\left(R \alpha ! \mathbf{C}_{\mathrm{G}_{1} \backslash \mathrm{G}_{2}}, \mathbf{C}_{\mathrm{G}_{3}[-p]}\right) \\
& \simeq \operatorname{Hom}\left(\mathbf{C}_{\mathrm{G}_{1} \backslash \mathrm{G}_{2}}, \alpha\left(\mathbf{C}_{\left.\left.\mathrm{G}_{3}[-p]\right)\right)},\right.\right. \\
& \simeq \operatorname{Hom}\left(\mathbf{C}_{\mathrm{G}_{1} \backslash \mathrm{G}_{2}}, \mathbf{C}_{\mathrm{G}_{1} \backslash \mathrm{G}_{2}}^{(1)}\right) \\
& \simeq \Gamma\left(\mathrm{G}_{1} \backslash \mathrm{G}_{2}, \mathbf{C}_{\mathrm{G}_{1} \backslash \mathrm{G}_{2}}^{(1)}\right)=0,
\end{aligned}
$$

where the fourth isomorphism uses the identification $\alpha^{!}(\cdot) \simeq \alpha^{-1}(\cdot)^{(1)}[p]$, due to the fact that $\alpha$ is smooth, and the relative orientation sheaf is non trivial. 


\section{§3.7. More on $S L(n+1, \mathbf{C})_{\mathbf{R}}$}

Using the table of Betti numbers for complex Grassmann manifolds, that we recall in Proposition 3.7.2, one gets the following sharpening of Proposition 3.4.2, which could be used to compute higher cohomology groups of the solution complex

$$
\operatorname{Sol}\left(\square_{m} \stackrel{D}{\otimes} \bar{\square}_{\widetilde{m}}, \mathcal{C}^{\infty}\left(\mathbf{C}_{\mathbb{G}_{\mathbf{R}}}\right)\right)
$$

Proposition 3.7.1. With the above notations, one has

$$
H^{k}\left(\mathbf{C}_{\mathbb{F} \times \overline{\mathbb{F}}} \circ \mathbf{C}_{\mathbb{G}_{\mathbf{R}}}\right) \simeq \mathbf{C}_{\mathbb{P}_{\mathbf{R}}}^{d(k-p ; n-p-1, p)} \oplus \mathbf{C}_{\mathbb{P} \times \overline{\mathbb{P}}}^{d(k ; n-p, p-1)} .
$$

Proof. Consider again the long exact cohomology sequence associated with (3.4.2). Using (3.4.3), (3.4.4) and Proposition 3.7.2 below, for $k \in \mathbf{Z}$ we get short exact sequences

$$
0 \rightarrow \mathbf{C}_{(\mathbb{P} \times \overline{\mathbb{P}}) \backslash \mathbb{P}_{\mathbf{R}}}^{d(k-p ; n-p-1, p)} \rightarrow \mathbf{C}_{\mathbb{P} \times \overline{\mathbb{P}}}^{d(k ; n-p, p)} \rightarrow H^{2 k}\left(\mathbf{C}_{\mathbb{F} \times \overline{\mathbb{F}}} \circ \mathbf{C}_{\mathbb{G}_{\mathbf{R}}}\right) \rightarrow 0,
$$

and also $H^{2 k+1}\left(\mathbf{C}_{\mathbb{F} \times \overline{\mathbb{F}}} \circ \mathbf{C}_{\mathbb{G}_{\mathbf{R}}}\right)=0$. An easy combinatorial argument, or the computation of the stalks of the above sequence at $\mathbb{P}_{\mathbf{R}}$ and $(\mathbb{P} \times \overline{\mathbb{P}}) \backslash \mathbb{P}_{\mathbf{R}}$, show that

$$
d(k ; n-p, p)=d(k-p ; n-p-1, p)+d(k ; n-p, p-1) .
$$

Proposition 3.7.2. $\quad$ (see e.g. [11]) Let $d(k ; m, q)$ be the partition function introduced in Proposition 3.6.3. Then, for $1 \leq q \leq m-1$, the Betti numbers of $\operatorname{Gr}\left(q ; \mathbf{C}^{m}\right)$ are given by

$$
\operatorname{dim} H^{k}\left(\operatorname{Gr}\left(q ; \mathbf{C}^{m}\right) ; \mathbf{C}_{\mathrm{Gr}\left(q ; \mathbf{C}^{m}\right)}\right)=d\left(\frac{k}{2} ; m-q, q\right) .
$$

\section{$\S$ A. Review on Projective Duality}

Projective duality is associated with the correspondence

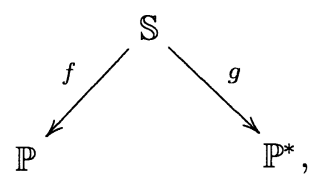


which is the extreme case of (1.1.1) obtained for $p=n-1$. In other words, one considers

$$
\begin{cases}\mathbb{P}=\operatorname{Gr}(1 ; \mathbb{V}) & \\ \mathbb{P}^{*}=\operatorname{Gr}(n ; \mathbb{V}) & \text { the family of hyperplanes in } \mathbb{P} \\ \mathbb{S}=\left\{(z, \zeta) \in \mathbb{P} \times \mathbb{P}^{*}: z \in \zeta\right\} & \text { the incidence relation. }\end{cases}
$$

In this section, we recall related results of [7,3], and we also discuss the action of $S U(\omega ; \mathbb{V})$, where $\omega$ is a nondegenerate hermitian form on $\mathbb{V}$, of signature $(q+1, n-q)$, for $1 \leq q \leq n-2$.

\section{§A.1. Adjunction formula}

Since $\mathbb{S} \subset \mathbb{P} \times \mathbb{P}^{*}$ is a smooth hypersurface, there are three perverse (resp. regular holonomic) kernels associated with the correspondence (A.0.1). More precisely, setting $\mathbb{S}^{c}=\left(\mathbb{P} \times \mathbb{P}^{*}\right) \backslash \mathbb{S}$ we will consider

(A.1.1) $\mathcal{K}=\left\{\begin{array}{l}\mathbf{C}_{\mathbb{S}[-1]} \\ \mathbf{C}_{\mathbb{S}^{c}} \\ \mathbf{C}_{\mathbb{S}^{c}}^{\prime}\end{array} \quad\right.$ and $\quad \mathfrak{K}=\left\{\begin{array}{l}\mathcal{B}_{\mathbb{S}} \\ \mathcal{B}_{\mathbb{S}^{c}} \\ \mathcal{B}_{\mathbb{S}^{c}}^{\vee}\end{array} \quad\right.$ for $\quad\left\{\begin{array}{l}-n-1<m<0 \\ m<0 \\ m>-n-1\end{array}\right.$

In particular, $\mathcal{B}_{\mathbb{S} c}$ is the sheaf of meromorphic functions with poles along $\mathbb{S}$, and $\mathcal{B}_{\mathbb{S}^{c}}^{\vee}=R \mathcal{H}$ om $_{\mathcal{D}_{\mathbb{P} \times \mathbb{P}^{*}}}\left(\mathcal{B}_{\mathbb{S}^{c}}, \mathcal{D}_{\mathbb{P} \times \mathbb{P}^{*}}\right) \otimes_{\mathcal{O}_{\mathbb{P} \times \mathbb{P}^{*}}} \Omega_{\mathbb{P} \times \mathbb{P}^{*}}^{\otimes-1}[2 n]$ denotes its dual as left $\mathcal{D}_{\mathbb{P} \times \mathbb{P}^{*} \text {-module. As pointed out in [22], one has }}$

$$
\mathbb{C}_{\mathbb{S}^{c}}^{\prime} \circ \mathbb{C}_{\mathbb{S}^{c}} \simeq \mathbf{C}_{\Delta[-2 n],} \mathcal{B}_{\mathbb{S}^{c}}^{\vee} \circ \mathcal{B}_{\mathbb{S}^{c}} \simeq \mathcal{B}_{\Delta},
$$

where $\Delta \subset \mathbb{P} \times \mathbb{P}$ denotes the diagonal. In particular, by (2.2.6) the functors $\mathbf{C}_{\mathbb{S}^{c}}^{\prime}[2 n] \circ \cdot$ and $\mathbf{C}_{\mathbb{S}} \circ \cdot\left(\right.$ resp. $\mathcal{B}_{\mathbb{S}^{c}}^{\vee} \stackrel{D}{\circ} \cdot$ and $\left.\cdot \stackrel{D}{\circ} \mathcal{B}_{\mathbb{S}^{c}}\right)$ are inverse to each other.

In this section, we have $p=n-1$. To avoid confusion with the notation $m^{*}=-m-p-2$, we set

$$
m^{\bullet}=-m-n-1
$$

One has the following analogue of Theorems 2.3.1 and 2.4.1.

Theorem A.1.1. (see $[7,8]$ ) Considering $\mathcal{K}$ and $\mathfrak{K}$ as in (A.1.1), the Radon correspondence (A.0.1) can be quantized to give a $\mathcal{D}_{\mathbb{P}}$-linear isomorphism

$$
\mathcal{D}_{\mathbb{P}^{*}(-m)} \stackrel{\sim}{\longrightarrow} \mathcal{D}_{\mathbb{P}}\left(-m^{\bullet}\right) \stackrel{D}{\circ} \mathfrak{K} .
$$

Moreover, for $\mathcal{G} \in \mathbb{D}_{\mathbf{R}-\mathrm{c}}^{\mathrm{b}}\left(\mathbf{C}_{\mathbb{P}^{*}}\right)$ and $\natural= \pm \infty$, $\pm \omega$ one has the isomorphism

$$
\mathrm{R} \Gamma\left(\mathbb{P} ; \mathcal{C}^{\natural}(\mathcal{K} \circ \mathcal{G})\left(m^{\bullet}\right)\right)[n] \simeq \mathrm{R} \Gamma\left(\mathbb{P}^{*} ; \mathcal{C}^{\natural}(\mathcal{G})_{(m)}\right) .
$$


As an example of application, note that an easy computation gives

$$
\mathbf{C}_{\mathbb{S}^{c}} \circ \mathbf{C}_{\mathbb{P}^{*}} \simeq \mathbf{C}_{\mathbb{P}[-2 n]}
$$

Then, for $\mathcal{G}=\mathbf{C}_{\mathbb{P}^{*}}$, one recovers Serre's isomorphism (3.3.8). Another example is obtained by considering $\mathcal{G}=\mathbf{C}_{\widehat{U}}$, for $U \subset \mathbb{P}$ "linéellement convexe" in the sense of Martineau. This is the analogue of Proposition 1.1.1 and was discussed in $[7$, Theorem 5.5].

\section{§A.2. Action of $S L(n+1, \mathbf{R})$}

As in Section 1.3.1, let $V$ be a real vector space of which $\mathbb{V}$ is a complexification. The closed orbit of the real form $S L(\mathrm{~V}) \subset S L(\mathbb{V})$ in $\mathbb{P}^{*}$ is identified with the real projective space $P^{*}$, dual to $P \subset \mathbb{P}$. The following result, which is the extreme case of Proposition 1.3.1, may be found for example in [12], for the $C^{\infty}$ case.

Proposition A.2.1. For $-n-1<m<0$ one has the isomorphism

$$
\Gamma\left(\mathrm{P} ; \mathcal{C}_{\mathrm{P}}^{\infty}\left(m^{\bullet} \mid \varepsilon^{\bullet}\right)\right) \stackrel{\sim}{\longrightarrow} \Gamma\left(\mathrm{P}^{*} ; \mathcal{C}_{\mathrm{P}^{*}}^{\infty}(m \mid \varepsilon)\right)
$$

and similar isomorphisms obtained when $\mathcal{C}^{\infty}$ is replaced by real analytic functions, distributions, or hyperfunctions.

As in [7], one may obtain this result as a corollary of Theorem A.1.1, using the isomorphism

$$
\mathbf{C}_{\mathbb{S}^{c}} \circ \mathbf{C}_{\mathbf{P}^{*}}^{(\varepsilon)} \doteq \mathbf{C}_{\mathbf{P}}^{\left(\varepsilon^{\bullet}\right)_{[-n]}}
$$

We refer to [3] for a proof along these lines of the analogue of Proposition 1.3.2. Here, the Cavalieri condition appears in describing the image of the Schwartz space $\mathcal{S}(\mathrm{A})$. For $n=3$ and $m^{\bullet}=-n$ this relates to the transform originally considered by Radon [25].

\section{§A.3. Action of $S U(q+1, n-q)$}

Let $1 \leq q \leq n-2$, and consider a nondegenerate hermitian form $\omega$ on $\mathbb{V}$ of signature $(q+1, n-q)$. The group $S U(\omega ; \mathbb{V}) \simeq S U(q+1, n-q)$ is a real form of $S L(\mathbb{V})$, and its orbits in $\mathbb{P}$ are

$$
\left\{\begin{array}{l}
\mathrm{P}=\left\{z \in \mathbb{P}:\left.\omega\right|_{z}=0\right\}, \quad \text { the set of } \omega \text {-isotropic lines, } \\
\mathrm{P}_{+}=\left\{z \in \mathbb{P}:\left.\omega\right|_{z}>0\right\} \\
\mathrm{P}_{-}=\left\{z \in \mathbb{P}:\left.\omega\right|_{z}<0\right\}
\end{array}\right.
$$


Similarly, recalling that $\zeta \in \mathbb{P}^{*}$ represents a hyperplane in $\mathbb{V}$, the orbits in $\mathbb{P}^{*}$ are

$$
\left\{\begin{array}{l}
\mathrm{P}^{*}=\left\{\zeta \in \mathbb{P}^{*}:\left.\omega\right|_{\zeta} \text { has signature }(q, n-q-1)\right\}, \\
\mathrm{P}_{+}^{*}=\left\{\zeta \in \mathbb{P}^{*}:\left.\omega\right|_{\zeta} \text { has signature }(q+1, n-q-1)\right\}, \\
\mathrm{P}_{-}^{*}=\left\{\zeta \in \mathbb{P}^{*}:\left.\omega\right|_{\zeta} \text { has signature }(q, n-q)\right\} .
\end{array}\right.
$$

Recall that $\mathrm{P}$ is a real quadratic hypersurface of $\mathbb{P}$ with non-degenerate Levi form of signature $(q, n-q-1)$, and similarly $\mathrm{P}^{*}$ has signature $(n-q-1, q)$. For $q=n-1$, the following result is the extreme case of Proposition 1.2.1.

Proposition A.3.1. For any $m \in \mathbf{Z}$ one has a commutative diagram

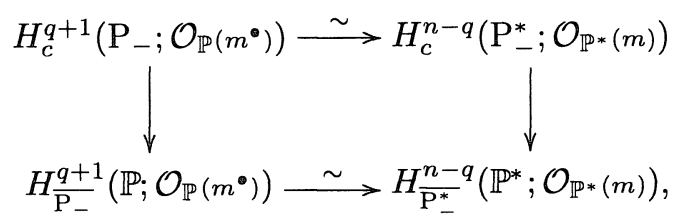

and the similar one obtained by replacing the - signs with + signs, and interchanging $q+1$ with $n-q$. In particular, for $q+1 \leq n-q$ one has a commutative diagram

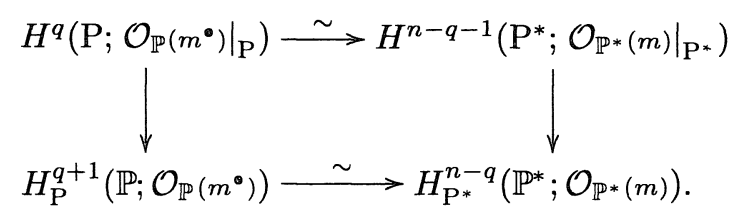

Proof. Note that if $m \geq 0$, then $m^{\bullet}<0$. Eventually interchanging the roles of $\mathbb{V}$ and $\mathbb{V}^{*}$, and of $q+1$ and $n-q$, it is not restrictive to assume that $m^{\bullet}<0$. Since the proofs are similar, we will just deal, for example, with the isomorphism

$$
H_{c}^{n-q}\left(\mathrm{P}_{+} ; \mathcal{O}_{\mathbb{P}}\left(m^{\bullet}\right)\right) \simeq H_{c}^{q+1}\left(\mathrm{P}_{+}^{*} ; \mathcal{O}_{\left.\mathbb{P}^{*}(m)\right)} .\right.
$$

Using Lemma A.3.2 below, Theorem A.1.1 for $\mathcal{K}=\mathbf{C}_{\mathbb{S}^{c}}^{\prime}, \mathcal{G}=\mathbf{C}_{\mathrm{P}_{+}^{*}}$ and $\emptyset=\omega$ gives

$$
\mathrm{R} \Gamma\left(\overline{\mathrm{P}_{-}} ; \mathcal{O}_{\mathbb{P}}\left(m^{\bullet}\right)\right)[n-q-1] \simeq \mathrm{R} \Gamma_{c}\left(\mathrm{P}_{+}^{*} ; \mathcal{O}_{\mathbb{P}^{*}}(m)\right)[q+1]
$$

Consider the distinguished triangle

$$
\mathrm{R} \Gamma_{c}\left(\mathrm{P}_{+} ; \mathcal{O}_{\mathbb{P}}\left(m^{\bullet}\right)\right) \rightarrow \mathrm{R} \Gamma\left(\mathbb{P} ; \mathcal{O}_{\mathbb{P}}\left(m^{\bullet}\right)\right) \rightarrow \mathrm{R} \Gamma\left(\overline{\mathrm{P}_{-}} ; \mathcal{O}_{\mathbb{P}}\left(m^{\bullet}\right)\right) \underset{+1}{\longrightarrow}
$$

associated with the short exact sequence $0 \rightarrow \mathbf{C}_{\mathrm{P}_{+}} \rightarrow \mathbf{C}_{\mathbb{P}} \rightarrow \mathbb{C}_{\overline{P_{-}}} \rightarrow 0$. By Serre's isomorphism (3.3.8), the middle term is in degree $n$ (or vanishes). It follows that

$$
H_{c}^{n-q}\left(\mathrm{P}_{+} ; \mathcal{O}_{\mathbb{P}}\left(m^{\bullet}\right)\right) \simeq H^{n-q-1}\left(\overline{\mathrm{P}_{-}} ; \mathcal{O}_{\mathbb{P}}\left(m^{\bullet}\right)\right)
$$


and the result follows by taking the zero-th cohomology group in (A.3.1).

Lemma A.3.2. One has

$$
\begin{array}{ll}
\mathbf{C}_{\overline{\mathrm{P}_{+}}} \circ \mathbf{C}_{\mathbb{S}^{c}} \simeq \mathbf{C}_{\mathrm{P}_{-}^{*}}[-2 q] & \mathbf{C}_{\overline{\mathrm{P}_{-}}} \circ \mathbf{C}_{\mathbb{S}^{c}} \simeq \mathbf{C}_{\mathrm{P}_{+}^{*}}[-2(n-q-1)] \\
\mathbf{C}_{\mathbb{S}^{c}}^{\prime} \circ \mathbf{C}_{\mathrm{P}_{-}^{*}} \simeq \mathbf{C}_{\overline{\mathrm{P}_{+}}}[-2(n-q)], & \mathbf{C}_{\mathbb{S}^{c}}^{\prime} \circ \mathbf{C}_{\mathrm{P}_{+}^{*}} \simeq \mathbf{C}_{\overline{\mathrm{P}_{-}}}[-2(q+1)] .
\end{array}
$$

Proof. By (A.1.2), the isomorphisms in the second line may be deduced from those in the first one. Since the arguments are similar we prove only the assertion

$$
\mathbf{C}_{\overline{\mathrm{P}_{+}}} \circ \mathbf{C}_{\mathbb{S}^{c}} \simeq \mathbf{C}_{\mathrm{P}_{-}^{*}[-2 q]} .
$$

Since the open orbit $\mathrm{P}_{-}^{*}$ is simply connected, if $\mathbf{C}_{\mathbb{S}^{c}} \circ \mathbf{C}_{\overline{\mathrm{P}_{+}^{*}}}$ is locally constant along $\mathrm{P}_{-}^{*}$, then it is constant. Thus, it is sufficient to show that

$$
\left(\mathbf{C}_{\overline{\mathrm{P}_{+}}} \circ \mathbf{C}_{\mathbb{S}^{c}}\right)_{\zeta} \simeq \mathrm{R} \Gamma_{c}\left((\mathbb{P} \backslash \widehat{\zeta}) \cap \overline{\mathrm{P}_{+}} ; \mathbf{C}\right) \simeq \begin{cases}\mathbf{C}_{[-2 q]} & \text { for } \zeta \in \mathrm{P}_{-}^{*} \\ 0 & \text { otherwise }\end{cases}
$$

Let $\zeta=[1,0, \ldots, 0]$ in a system $\left[\zeta_{0}, \ldots, \zeta_{n}\right]$ of homogeneous coordinates in $\mathbb{P}^{*}$, and denote by $\left[z_{0}, \ldots, z_{n}\right] \in \mathbb{P}$ the dual system in $\mathbb{P}$. Note that $\mathbb{P} \backslash \widehat{\zeta}$ is the affine chart $z_{0} \neq 0$. We endow it with the system of affine coordinates $\left(\tau_{1}, \tau^{\prime}, \tau^{\prime \prime}\right) \in \mathbf{C}^{1+q+(n-q-1)}$, where $\tau_{j}=z_{j} / z_{0}$.

First, let $\zeta \in \mathrm{P}^{*}$. Then, we may assume

$$
\omega=\left(\begin{array}{cccc}
0 & 1_{1} & 0 & 0 \\
1_{1} & 0 & 0 & 0 \\
0 & 0 & 1_{q} & 0 \\
0 & 0 & 0 & -1_{n-q-1}
\end{array}\right) .
$$

In this case,

$$
(\mathbb{P} \backslash \widehat{\zeta}) \cap \overline{\mathrm{P}_{+}} \simeq\left\{\left(\tau_{1}, \tau^{\prime}, \tau^{\prime \prime}\right): 2 \operatorname{Re} \tau_{1} \geq-\left|\tau^{\prime}\right|^{2}+\left|\tau^{\prime \prime}\right|^{2}\right\}
$$

is isomorphic to an affine closed half-space of $\mathbf{R}^{2 n}$, and hence $R \Gamma_{c}((\mathbb{P} \backslash \widehat{\zeta}) \cap$ $\left.\overline{\mathrm{P}_{+}} ; \mathbf{C}\right)=0$.

Finally, let $\zeta \in \mathrm{P}_{ \pm}^{*}$. Then, we may assume

$$
\omega=\left(\begin{array}{cccc}
\mp 1_{1} & 0 & 0 & 0 \\
0 & \pm 1_{1} & 0 & 0 \\
0 & 0 & 1_{q} & 0 \\
0 & 0 & 0 & -1_{n-q-1}
\end{array}\right)
$$

Since

$$
(\mathbb{P} \backslash \widehat{\zeta}) \cap \overline{\mathrm{P}_{+}} \simeq\left\{\left(\tau_{1}, \tau^{\prime}, \tau^{\prime \prime}\right): \pm\left|\tau_{1}\right|^{2}+\left|\tau^{\prime}\right|^{2}-\left|\tau^{\prime \prime}\right|^{2} \mp 1 \geq 0\right\}
$$


one concludes using Lemma A.3.3 below.

Lemma A.3.3. Let $\left(x^{\prime}, x^{\prime \prime}\right) \in \mathbf{R}^{r+s}$ be a system of coordinates, and consider the sets

$$
\Sigma_{r, s}^{\geq}=\left\{\left(x^{\prime}, x^{\prime \prime}\right):\left|x^{\prime}\right|-\left|x^{\prime \prime}\right| \geq 1\right\}, \quad \Sigma_{r, s}^{\leq}=\left\{\left(x^{\prime}, x^{\prime \prime}\right):\left|x^{\prime}\right|-\left|x^{\prime \prime}\right| \leq 1\right\} .
$$

Then,

$$
\mathrm{R} \Gamma_{c}\left(\Sigma_{r, s}^{\geq} ; \mathbf{C}\right)=0, \quad \mathrm{R} \Gamma_{c}\left(\Sigma_{r, s}^{\leq} ; \mathbf{C}\right) \simeq \mathbf{C}[-s]
$$

Proof. Setting $\Sigma_{r, s}^{=}=\left\{\left(x^{\prime}, x^{\prime \prime}\right):\left|x^{\prime}\right|-\left|x^{\prime \prime}\right|=1\right\}$, one has

$$
\mathrm{R} \Gamma_{c}\left(\Sigma_{r, s}^{\geq} ; \mathbf{C}\right) \simeq \mathrm{R} \Gamma_{c}\left(\Sigma_{r, s}^{=} ; R \gamma_{!} \mathbf{C}_{\Sigma_{r, s}^{2}}^{\geq}\right),
$$

where $\gamma: \Sigma_{\bar{r}, s}^{>} \rightarrow \Sigma_{r, s}^{=}$is the natural homothety. The fibers of $\gamma$ are closed half lines $\{t \in \mathbf{R}: t \geq 1\}$, and hence $R \gamma_{!} \mathbf{C}_{\Sigma_{r, s}}^{\geq}=0$. Concerning $\Sigma_{r, s}^{\leq}$, the projection $\left(x^{\prime}, x^{\prime \prime}\right) \mapsto x^{\prime}$ gives it a structure of a vector bundle over the closed unit ball $B^{r} \subset \mathbf{R}^{r}$. Since $B^{r}$ is contractible, $\Sigma_{r, s}^{\leq}$is isomorphic to $B^{r} \times \mathbb{R}^{s}$, and hence

$$
\mathrm{R} \Gamma_{c}\left(\Sigma_{r, s}^{\leq} ; \mathbf{C}\right) \simeq \mathrm{R} \Gamma_{c}\left(B^{r} ; \mathbf{C}\right)[-s] \simeq \mathbf{C}[-s]
$$

\section{References}

[1 1 Bailey, T., Ehrenpreis, L. and Wells, R. O., Jr., Weak solutions of the massless field equations, Proc. Roy. Soc. London Ser. A, 384 (1982), no. 1787, 403-425.

[2] Baston, R. J. and Eastwood, M. G., The Penrose transform. Its interaction with representation theory, The Clarendon Press Oxford University Press, New York, 1989.

[3] D'Agnolo, A., Radon transform and the Cavalieri condition: a cohomological approach, Duke Math. J., 93 (1998), 597-632.

[4] , Sheaves and $\mathcal{D}$-modules in integral geometry, Analysis, Geometry, Number Theory: The Mathematics of Leon Ehrenpreis, Contemp. Math., 251 (2000).

[5] D'Agnolo, A. and Marastoni, C., A family of Radon D-modules, Preprint, (1999).

[6] D'Agnolo, A. and Schapira, P., Radon-Penrose transform for D-modules, J. Funct. Anal., 139 (1996), 349-382.

[7] L Leray's quantization of projective duality, Duke Math. J., 84 (1996), 453-496.

[8] The Radon-Penrose correspondence. II. Line bundles and simple $\mathcal{D}$-modules, $J$. Funct. Anal., 153 (1998), 343-356.

[9] Eastwood, M. G., Complex methods in real integral geometry (with the collaboration of T. N. Bailey and C. R. Graham), Proceedings of the 16th Winter School "Geometry and Physics" (Srní, 1996), Rend. Circ. Mat. Palermo (2) Suppl., 46 (1997), 55-71. 
[10] Eastwood, M. G., Penrose, R. and Wells, R. O., Jr., Cohomology and massless fields, Comm. Math. Phys., 78 (1980/81), 305-351.

[11] Fuks, D. B., Classical manifolds, Current problems in mathematıcs. Fundamental directions, 12 (Russian), Itogi Nauki i Tekhnıki, (1986), 253-314, 322.

[12] Gelfand, I. M., Gindikin, S. G. and Graev, M. I., Integral geometry in affine and projective spaces, J. Soviet Math., 18 (1982), 39-167.

[13] Gindikin, S. G. and Henkin, G. M., Integral geometry for $\bar{\partial}$-cohomology in $q$-linearly concave domains in $\mathbb{C P}^{n}$, Funktsional. Anal. i Prilozhen, 12 (1978), 6-23.

[14] Guillemin, V., The integral geometry of line complexes and a theorem of Gelfand-Graev, Astérısque, hors série (1985), 135-149. The mathematical heritage of Élie Cartan (Lyon, 1984).

[15] Helgason, S., Groups and geometric analysis. Integral geometry, invariant differential operators, and spherical functions, Pure Appl. Math., 113 (1984), Academic Press.

[16] Henkin, G. M. and Polyakov, P. L., Homotopy formulas for the $\bar{\partial}$-operator on $\mathbb{C P}^{n}$ and the Radon-Penrose transform, Izv. Math., 3 (1987), 555-587.

[17] John, F., The ultrahyperbolic differential equation with four independent variables, Duke Math. J., 4 (1938), 300-322, reprinted in 75 years of Radon transform (Vienna, 1992), Conf. Proc. Lecture Notes Math. Phys., IV, 1994.

[18] Kashiwara, M., Algebraic study of systems of partial differential equations, Mém. Soc. Math. Fr. (N.S.), 123 (1995), fasc. 4 no. 63, xiv+72, Translated from the Japanese by A. D'Agnolo and J.-P. Schneiders.

[19j _ The Riemann-Hilbert problem for holonomic systems, Publ. RIMS, Kyoto Univ., 20 (1984), 319-365.

[20] Kashiwara, M. and Schapira, P., Sheaves on manifolds, Grundlehren Math. Wiss., 292 (1990), Springer-Verlag, Berlin.

[21] , Moderate and formal cohomology associated with constructible sheaves, Mém. Soc. Math. Fr. (N.S.), 124 (1996), fasc. 1 no. 64 , iv +76.

[22] Kashiwara, M. and Tanisaki, T., Kazhdan-Lusztig conjecture for affine Lie algebras with negative level. II. Nonintegral case, Duke Math. J., 84 (1996), 771-813.

[23] Marastoni, C., Grassmann duality for D-modules, Ann. Scr. École Norm. Sup. (4), 31 (1998), 459-491.

[24] Oshima, T., Generalized Capelli identities and boundary value problems for $G L(n)$, Structure of solutions of differential equations (Katata/Kyoto, 1995), World Sci. Publishing, River Edge, NJ, 1996, pp. 307-335.

[25] Radon, J., Über die bestimmung von funktionen durch ihre integralwerte längs gewisser mannigfaltigkeiten, Ber. Verh. Konigl. Sachs. Ges. Wiss. Leipzig 69 (1917), 262-277, reprinted in 75 years of Radon transform (Vienna, 1992), Conf. Proc. Lecture Notes Math. Phys., IV, 1994.

[26] Sekiguchi, H., The Penrose transform for certain non-compact homogeneous manifolds of U $(n, n)$, J. Math. Scr. Univ. Tokyo, 3 (1996), 655-697.

[27] Tanisaki, T., Hypergeometric systems and Radon transforms for hermitian symmetric spaces, Adv. Stud. Pure Math. (T. Kobayashi et al., eds.), 26 (2000), MSJ.

[28] Wells, R. O., Jr., Hyperfunction solutions of the zero-rest-mass field equations, Comm. Math. Phys., 78 (1980/81), 567-600. 
